\title{
ON THEORIES OF SOLAR ROTATION
}

\author{
B. R. DURNEY \\ National Center for Atmospheric Research* \\ Boulder, Colo. 80303, U.S.A.
}

\section{Foreword}

About twelve years ago the first attempts to explain the Sun's differential rotation appeared in the literature. In these twelve years our understanding of the processes that could give rise to the large-scale circulation of the solar convection zone has greatly increased.

It therefore seems appropriate at this time to critically review the degree of development of theories of solar rotation. This could perhaps be achieved by referring the reader to the relevant papers. In this type of review the reader must, however, spend a considerable effort to gain a proper understanding of the subject. Consequently, in this paper a different approach has been followed, the aim of which has been to present a unified and critical exposition of theories of solar rotation. There is no doubt that this paper would be very different had it been written by another contributor to this field: a subject can be understood in several distinct ways; furthermore, different authors would weight very differently the diverse theories that are put forward to explain the Sun's differential rotation since there does not exist at present a commonly accepted explanation of this phenomenon.

This review will have achieved its goal if it allows the reader to gain an easy understanding of this subject and if it clearly shows where the main difficulties lie.

\begin{abstract}
The main theories of solar rotation are critically reviewed.
The interaction of large-scale convection with rotation gives rise to a transport of angular momentum towards the equator and therefore to differential rotation with equatorial acceleration. (Large-scale convection is defined as follows: in a highly turbulent fluid, the small-scale turbulence acts as a viscosity and organizes fluid motions on a much larger scale.) This transport of angular momentum towards the equator arises because of the highly non-axisymmetric character of the large-scale convective motions in the presence of rotation. These motions tend to be concentrated near the equator. It is not surprising, therefore, that for magnitudes of large-scale convection which are needed to generate the observed solar differential rotation, large and unobserved pole-equator differences in flux appear in the Boussinesq approximation.

It is important, therefore, to take the variations in density into account. Studies of large-scale convection in a compressible rotating medium are still in a very early stage; these studies suggest, however, that the surface layers must indeed rotate differentially.

The interaction of rotation with convection appears to be especially efficient in generating a poleequator difference in flux, $\Delta \mathscr{F}$. Such a $\Delta \mathscr{F}$ drives meridional motions, and the action of Coriolis forces on these motions gives rise to differential rotation. In the 'large-viscosity' approximation the problem separates; the meridional motions can be determined first (from the radial and latitudinal equations of motions, and the energy equation) and the angular velocity can be determined next from the azimuthal equation of motion. Since very little is known about compressible large-scale convection, it has been assumed in the development of this theory that the stabilizing effect of rotation on turbulent convection depends on the polar angle $\theta$ and on depth. The solution for the angular velocity in the large viscosity approximation gives a differential rotation that varies slowly with depth. As a consequence, the large
\end{abstract}

* The National Center for Atmospheric Research is sponsored by the National Science Foundation. 
viscosity approximation is not valid over most of the convection zone, the Coriolis term being larger than the viscous term; a thin layer at the top excepted. (It appears, however, that if the angular velocity, $\Omega$, is a slowly varying function of depth and the azimuthal stresses vanish at both ends of the convection zone, then the general behavior of $\Omega$ will be very much like that predicted by the large viscosity approximation.)

The stabilizing effect of rotation on turbulent convection is neglected; if differential rotation is significant over the entire convection zone, and if the meridional and large-scale convective velocities are not too large, then in the radial and latitudinal equations of motion, the main balance of forces is between pressure gradients, buoyancy and Coriolis forces. If rotation is not constant along cylinders, then the differential rotation gives rise to latitudinal variations in the convective flux which are proportional to $\Omega_{0}^{2} T / g$ (where $T$ is the temperature and $g$ is gravity). In the lower part of the convection zone, $\Omega_{0}^{2} T / g$ is of the order of the superadiabatic gradient itself. Therefore large pole-equator differences in flux, $\Delta \mathscr{F}$, will be present unless the angular velocity is constant along cylinders. The meridional velocities associated with this rotation law are not small, however, and could generate a significant $\Delta \mathscr{F}$. It could well be that large $\Delta$ F's can be avoided only if rotation is uniform in the lower part of the convection zone. (To be certain of these results, however, it is important to estimate the magnitude of the stabilizing effect of rotation on turbulent convection.)

Turbulent convection is driven by the buoyancy force which thus introduces a preferred direction: gravity. In consequence, the turbulence in the sun should be anisotropic and if this is the case the convection zone cannot rotate uniformly. The degree of anisotropy is not known and must be determined from the observed solar differential rotation. The anisotropy is such that the horizontal exchange of momentum is larger than the vertical.

The normal mode of vibrations and the inner rotation of the Sun are briefly discussed.

\section{Observations}

The observations that have a bearing on theories of solar rotation are the following.

\subsection{RotATION RATES}

Different features of the solar surface rotate at various rates. In Figure 1 (from Stenflo, 1974) the angular velocity is plotted as a function of latitude for (i) the sunspots (Newton and Nunn, 1951), (ii) the photosphere measured from Doppler shifts (Howard and Harvey, 1970), (iii) the photospheric magnetic field measured by an auto correlation technique (Wilcox and Howard, 1970; Wilcox et al., 1970a; Stenflo, 1974). For the curve labeled 'longitudinal magnetic field' Stenflo calculated the autocorrelation curves for the strength (with sign) of the radial component of the field. Only the sign was used in the curve labeled 'sign of the longitudinal magnetic field.' To some extent the dashed, solid and dotted curves of Figure 1 correspond to increasing values of the flux density.

The chromosphere and transition region appear to rotate like the photosphere (Dupree and Henze, 1972; Henze and Dupree, 1973). The results of OSO 6 (Henze and Dupree) indicate a smaller differential rotation than the data of OSO 4 (Dupree and Henze). If only the brightest points in the EUV spectroheliograms are included, then their rotation rate is similar to that of the sunspots (Simon and Noyes, 1972).

Measurements of the chromospheric rotation rate in the $\mathrm{H} \alpha$ line give larger angular velocities than when metallic lines are used (Livingston, 1969). The Kcorona rotates as the sunspots (Hansen et al., 1969).

The above data have been interpreted by Stenflo (1974) as indicating that observations which utilize the Doppler and Zeeman effects refer to different regions of the Sun: the Doppler shift measurements refer to non-magnetic regions which do not contribute to the Zeeman-effect observations. Furthermore, Stenflo suggests 


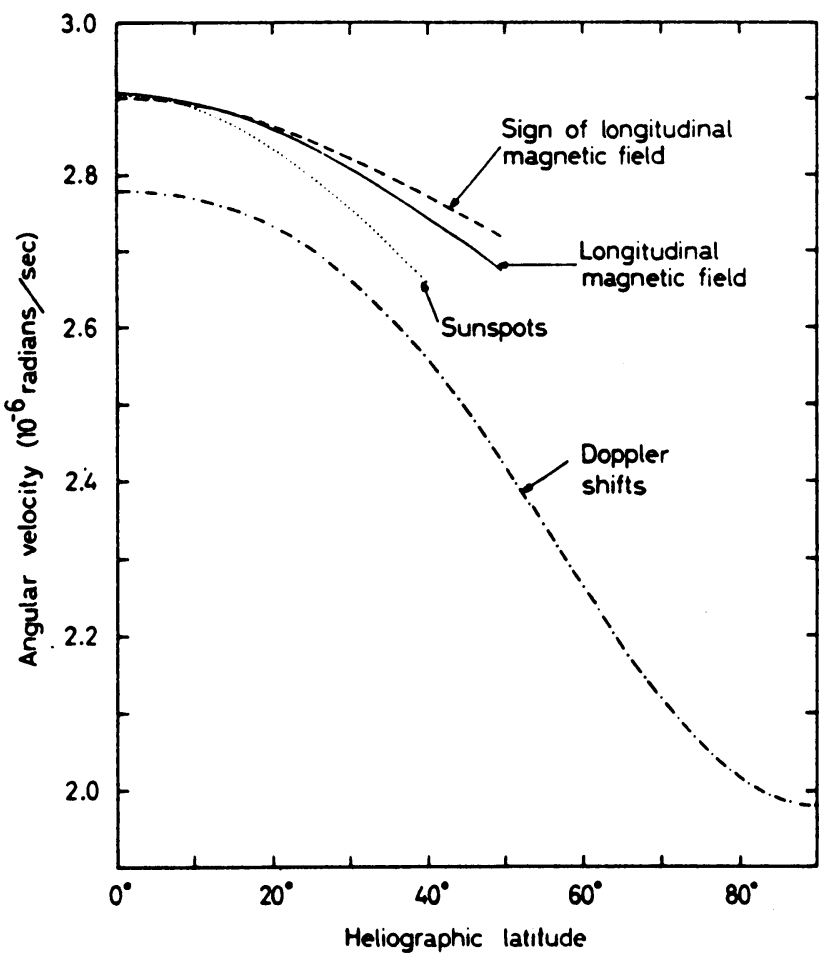

Fig. 1. Angular velocity of rotation as a function of heliographic latitude. The solid and dashed lines are from Stenflo (1974), the curve for recurrent sunspots is from Newton and Nunn (1951), and the Doppler shift curve is from Howard and Harvey (1970) (from Stenflo: 1974, Solar Phys. 36, 495).

that the smaller the differential rotation of the magnetized plasma, the deeper the magnetic field pattern is rooted. It will be seen later that this picture is not without difficulties.

\subsection{Time VARIATIONS AND CORRELATIONS}

Howard and Harvey expressed the Sun's angular velocity in the form

$$
\Omega=a+b \cos ^{2} \theta+c \cos ^{4} \theta
$$

where $\theta$ is the polar angle. The average values of $a, b$, and $c$ were found to be: $a=2.78 \times 10^{-6} \mathrm{rad} \mathrm{s}^{-1}, \quad b=-3.51 \times 10^{-7} \mathrm{rad} \mathrm{s}^{-1}$, and $c=-4.43 \times 10^{-7} \mathrm{rad} \mathrm{s}^{-1}$. Time variations of $a, b$, and $c$ are large and correlations exist between these time variations (cf. Howard and Harvey, 1970). In Figure 2 we have plotted the correlation between $b$, and $c$ as given by Yoshimura (1972a). A weaker correlation exists between $a$ and $c$ (Wolff, 1975).

\subsection{RIGID ROTATION, THE SECTOR STRUCTURE AND PREFERRED LONGITUDES}

The interplanetary sector structure (Wilcox and Ness, 1965) has been shown to be an extension of the solar sector structure (Ness and Wilcox, 1966; Wilcox and Ness, 


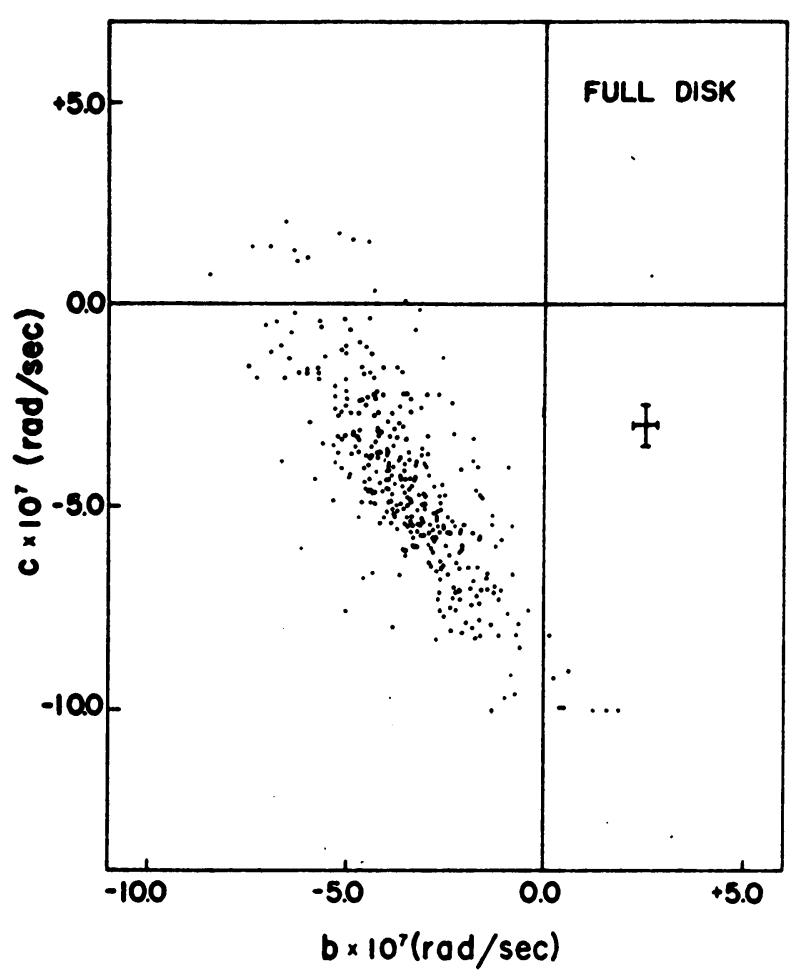

Fig. 2. A plot of the values of $b$ versus $c$ for the whole disk. The values of $b$ and $c$ are from Howard and Harvey (1970) (from Yoshimura: 1972, Solar Phys. 22, 20).

1967). Boundaries of the solar sector structure do not evolve as expected from the solar differential rotation, but appear to have a rigid-rotation component (Wilcox and Howard, 1968). Large-scale photospheric magnetic fields can also display, at some latitudes, both rigid and differential rotation properties (Wilcox et al., 1970b). Švestka $(1968 \mathrm{a}, \mathrm{b})$ has found that the sources of proton flare rotate rigidly around the Sun in the opposite direction of rotation. Also, the long-lived coronal activity shows rigid rotation in the latitude interval $\pm 57.5^{\circ}$ (Antonucci and Svalgaard, 1974).

Flare activity, especially the proton flare activity, occurs preferentially near the sector boundaries (Bumba and Obridko, 1969); a marked enhancement is found within one day of the $(-+)$ solar sector boundaries (Dittmer, 1975).

\subsection{GIANT MAGNETIC FIELD STRUCTURES, MERIDIONAL MOTIONS AND ANGULAR MOMENTUM TRANSPORT}

Giant magnetic field structures have been observed by Bumba et al. (1964), Bumba (1967), McIntosh (1975) and others. Their possible origin has been discussed by Bumba (1970).

From spectroscopic measurements, Howard (1971) finds no evidence of meridional motions in the photosphere with an upper limit to the line-of-sight velocity of $30 \mathrm{~m} \mathrm{~s}^{-1}$. Sunspots, on the other hand, show a poleward drift of about $0.01 \mathrm{deg} \mathrm{day}^{-1}$ 
for latitudes higher than $\sim 20^{\circ}$ and a drift towards the equator for smaller latitudes (Tuominen, 1955; Richardson and Schwarzschild, 1953).

From a statistical analysis, Ward (1965) found that the longitudinal and latitudinal components of the proper velocities of the sunspots are correlated: $\left.\left\langle U_{\phi} U_{\theta}\right\rangle\right\rangle 0$, that is, on the average the sunspots with $U_{\phi}-\left\langle U_{\phi}\right\rangle>0$ move towards the equator (and those with $U_{\phi}-\left\langle U_{\phi}\right\rangle<0$ towards the pole), giving rise to a transport of angular momentum towards the equator (cf. Starr and Gilman, 1965). Ward's results have been criticized by Leighton (1966) and until further evidence becomes available, the correlation $\left\langle U_{\phi} U_{\theta}\right\rangle>0$ cannot be considered as established.

\subsection{Pole-eQuator difFERENCES IN FLUX AND TEMPERATURE}

The pole-equator differences in flux $(\Delta \mathscr{F})$ are very small (Dicke and Goldenberg, 1967; Hill et al., 1974); the upper limit of $\Delta \mathscr{F} / \mathscr{F}$ is probably not larger than a few parts in $10^{-4}$. Pole-equator differences in temperature $(\Delta T)$, if present, are also small (Appenzeller and Schröter, 1967; Caccin et al., 1970; Altrock and Canfield, 1972a, 1972b; Noyes et al., 1973; Rutten, 1973; Falciani et al., 1974). Noyes et al. (1973) find that $\Delta T \cdot \tau \leq 0.3 \mathrm{~K}$ if $\tau<10^{-2}$ ( $\tau$ is the optical depth).

\subsection{NORMAL MODE OF VIBRATIONS}

Evidence has been accumulating (Deubner, 1972; Kaufman, 1972; Kobrin and Korshunov, 1972; Fossat and Ricort, 1973) that normal modes of vibration of the entire Sun are present. (Fossat (1975) has, however cautioned that the long-period oscillation of the data in Fossat and Ricort's paper could be contaminated by atmospheric noise.) Recent observations by the SCLERA group (cf. Hill et al., 1976) dispel any doubt about the existence of these normal modes of vibration.

It is unlikely that observations of solar-type stars, following their arrival at the main sequence, could be relevant for theories of the solar differential rotation. These observations are essential, however, for theories of the solar inner rotation (the radiative core).

\subsection{Average Rotational Velocities of Main Sequence Stars}

The average rotational velocity for stars in the main sequence increases from $\sim 180 \mathrm{~km} \mathrm{~s}^{-1}$ for a B0 star to $\sim 225 \mathrm{~km} \mathrm{~s}^{-1}$ for a B5 star and decreases thereafter $\left(\sim 100 \mathrm{~km} \mathrm{~s}^{-1}\right.$ for an F0 star), the decrease becoming very sharp at about F6 (Abt and Hunter, 1962). Figure 3 (from Kraft, 1969) shows the log of the average angular momentum density versus the log of the mass for stars in the main sequence $(\langle\mathrm{J}\rangle$ is the average angular momentum, assuming solid body rotation, of main-sequence stars with a given mass, divided by the star's mass $M)$. The extrapolated line, $\langle J\rangle \sim M^{2 / 3}$, was added by Dicke (1970a, b).

\subsection{Angular Velocity as a Function of Age and Ca II Emission}

Stars later than F6 rotate faster in young than in old clusters (Kraft, 1967). Figure 3 shows that rotation rate becomes very small in the main sequence for stars later than $\sim$ F6. It is just at this place that Ca II emission begins (Wilson, 1966a, 1966b). 


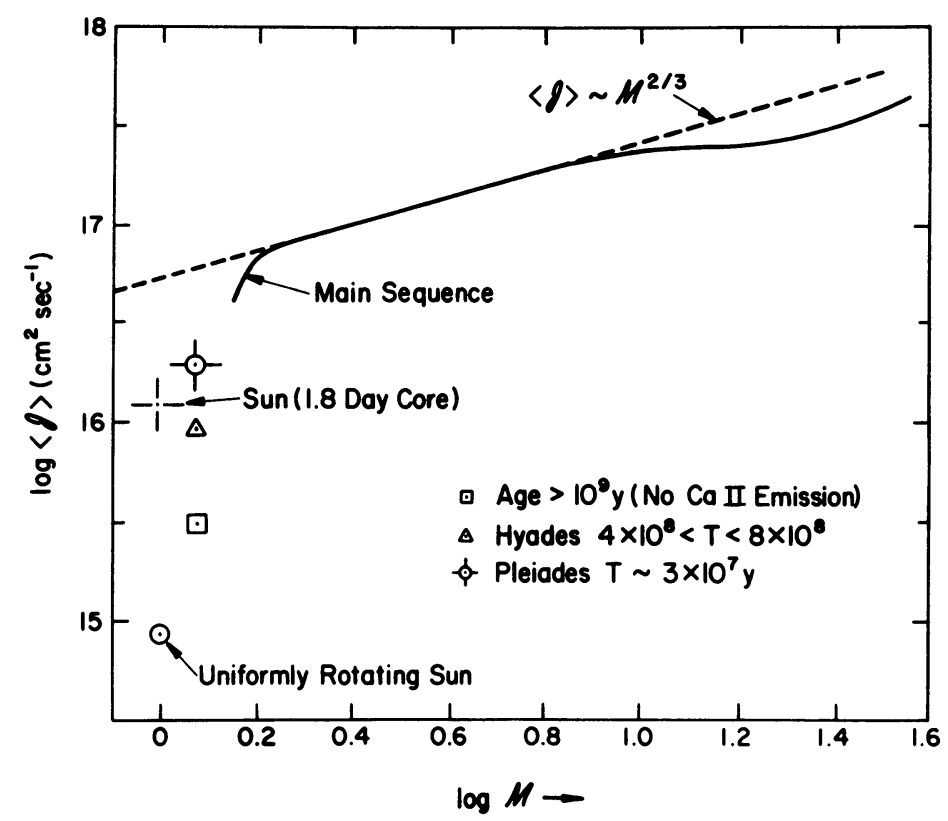

Fig. 3. The log of the angular momentum, per unit mass, of stars of various masses (assumed to be rotating uniformly) versus the log of the mass (from Kraft, 1969, in Stellar Astronomy, vol. 2 (ed. by Chiu, Warasila and Remo), Gordon and Breach, New York.

Furthermore, Wilson (1966b) has shown that stars with Ca II emission are nearer the zero-age main sequence than stars without it. Figure 4 (from Kraft, 1967) shows that, for stars less massive than $M / M_{\odot}=1.25$, the largest rotational velocities are associated with stars having active chromospheres (Ca II emission). For more details about the above observations the reader is referred to the excellent review articles by $\operatorname{Kraft}(1969,1970)$.

\subsection{Lithium and Beryllium Abundances}

There is a correlation between the $\mathrm{Li}$ abundance and the age of the star: the older the star, the lower the $\mathrm{Li}$ abundance; that is, there is a progressive loss of $\mathrm{Li}$ with time (cf. Wallerstein and Conti, 1969).

Stars with masses less than $1.1 M_{\odot}$ have much lower abundance of $\mathrm{Li}$ than stars with $M>1.1 M_{\odot}$ (Wallerstein et al., 1965). Stars with appreciable rotation have the largest $\mathrm{Li}$ content (Conti, 1968).

The abundances of beryllium show a much lower dispersion than the abundances of $\mathrm{Li}$ (cf. Wallerstein and Conti, 1969; Grevesse, 1968; Hauge and Engvold, 1968; Ross and Aller, 1974). Ross and Aller find that, in the photospheric layers of the Sun, beryllium is depleted below the solar system abundance by a factor of about two. It is not possible, therefore, to rule out a small depletion of $\mathrm{Be}$ in the Sun. In contrast, solar-type stars show a very strong depletion of lithium.

Lithium and beryllium are destroyed at distances from the center of the Sun equal to $\sim 0.63 R_{\odot}$ and $\sim 0.47 R_{\odot}$, respectively (cf. Dicke, 1970b, Table 2). In relation to 


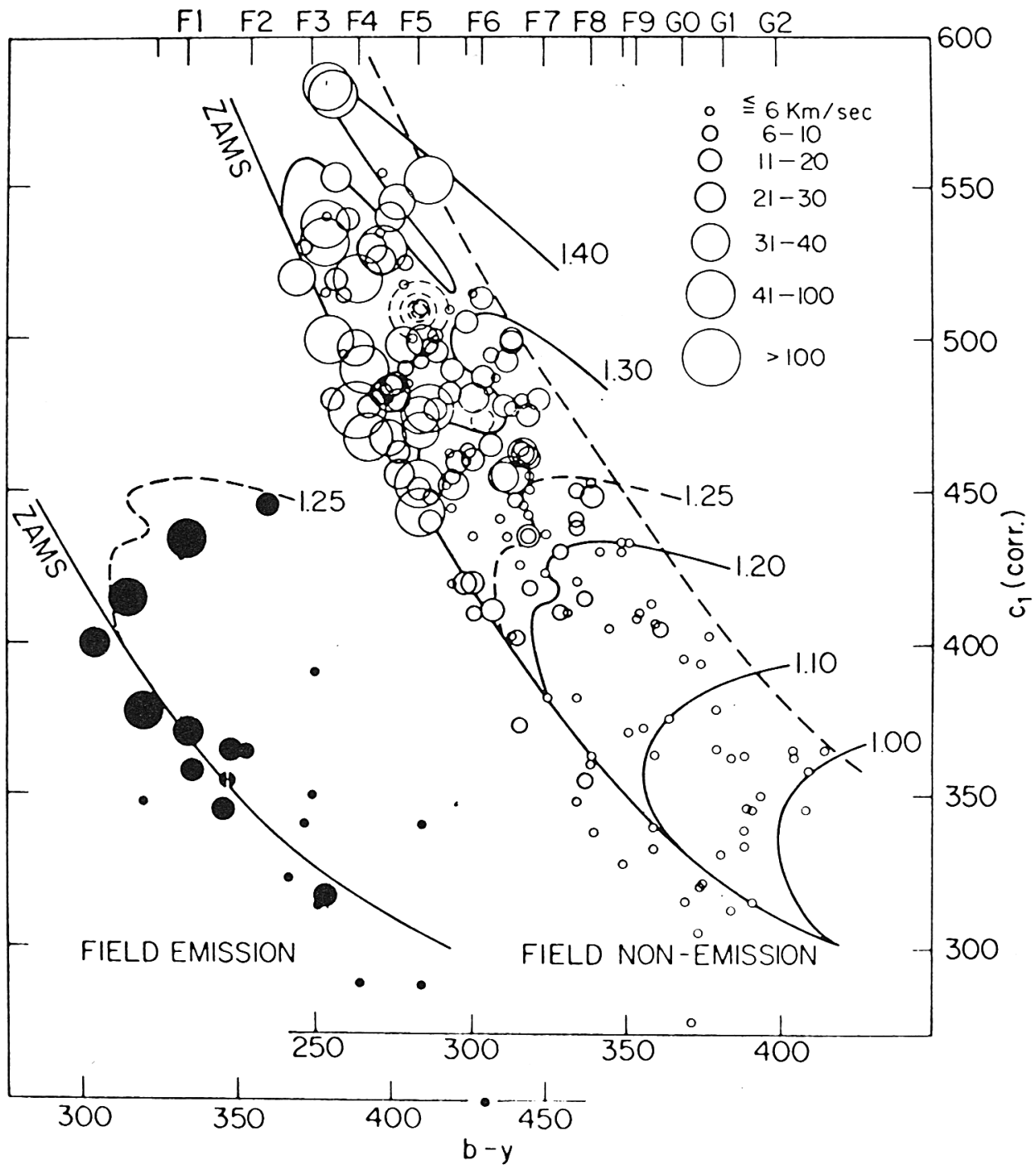

Fig. 4. Strömgren diagram representation of the HR diagram for both field emission stars (dark circles) and field-free emission stars (open circles). The circle size gives an indication of the rotation rate (from Kraft: 1967, Astrophys. J. 150, 551).

theories of the solar rotation, the importance of these observations lies in the fact that they indicate that there is very little mixing of matter from the surface down to $0.47 R_{\odot}$. To account for the Li depletion, however, a mixed region must exist, extending down to $0.63 R_{\odot}$. This mixing has been attributed to turbulence induced by gradients in the angular velocity (Goldreich and Schubert, 1967; Spiegel, 1968; Howard et al., 1967). 


\section{Differential Rotation as a Consequence of the Interaction of Rotation with Convection}

\subsection{BOUSSINESQ THEORIES}

Typical values of the Rayleigh $(\mathscr{R})$ and Prandtl $(\sigma)$ numbers in the solar convection zone are $\mathscr{R} \sim 10^{12}$ to $10^{20}$ (depending mainly on whether one takes the length appearing in $\mathscr{R}$ as a scale height or as the depth of the convection zone; cf. Spiegel, 1971) and $\sigma \sim 10^{-9}$ because the thermal diffusion is radiative (Ledoux et al., 1961). Convection in the Sun should therefore be highly turbulent.

An idea that goes back to the beinning of turbulence studies (Boussinesq, 1877, 1897 ) is that of a turbulent viscosity: the momentum exchange due to the turbulent motions is assumed to act as a viscosity and to organize relatively steady large-scale motions. The effect of rotation on this large-scale convection will be especially important and this leads to an appealing and natural theory of differential rotation: the radial and latitudinal variations of the angular velocity are assumed to be generated by the interaction of this global convection with rotation (Durney, 1968a, 1970; Busse, 1970, 1973; Yoshimura and Kato, 1971). As a starting point, let us ignore the compressible character of the solar convection zone and consider a spherical layer of a rotating convective fluid in the Boussinesq approximation. The usual boundary conditions imposed on this problem are specified temperatures at the top $\left(R_{0}\right)$ and bottom $\left(R_{\mathrm{c}}\right)$ of the convection zone, as well as zero stresses and radial velocities at $r=R_{0}$ and $r=R_{\mathrm{c}}$. These boundary conditions are very far from being ideal. Assuming that the flow problems in the inner radiative region and in the convective zone can be separated (and it could well be that this is not possible), it seems more appropriate to specify the energy flux and the temperature at the inner boundary of the convection zone, letting the outer surface choose its own temperature and flux. In particular, a boundary condition of uniform heat flux at $r=R_{\mathrm{c}}$ could significantly alter the nature of the solutions. Furthermore, the boundary conditions of zero stresses at $r=R_{\mathrm{c}}$ could be questioned (Gierasch, 1974; Durney, 1976).

We define dimensionless quantities as follows:

$$
\begin{aligned}
& \mathbf{U}=\kappa U^{\prime} / R_{0} ; \quad \mathbf{r}=R_{0} \mathbf{r}^{\prime} ; \quad t=R_{0}^{2} t^{\prime} / \kappa ; \\
& T=\Delta T T^{\prime} ; \quad \mathbf{G}(r)=-g(r) \hat{r}=-g\left(R_{0}\right) g^{\prime}\left(r^{\prime}\right) \hat{r}^{\prime}
\end{aligned}
$$

$\hat{r}$ and $\hat{r}^{\prime}$ are unit vectors and the primed quantities are the dimensionless variables for the velocity, radial distance, time, temperature, and gravity $(\mathbf{G}(r))$, respectively. (If $M_{0}$ is the mass of the Sun and if we neglect the mass of the convection zone then $g\left(R_{0}\right)=G_{\mathrm{c}} M_{0} / R_{0}^{2}$ and $g^{\prime}\left(r^{\prime}\right)=1 / r^{2} ; G_{\mathrm{c}}$ is the gravitational constant). In Equations (2.1.1), $\kappa$ is the thermometric diffusivity and $\Delta T=T_{c}-T_{0}$, that is, the difference in temperature between the inner and outer surfaces of the convection zone. In the rest of this section all quantities will be assumed to be dimensionless and the primes will be dropped.

Differential rotation is an axisymmetric mode of the velocity field $\mathbf{U}$. To understand its origin it is convenient to expand the velocity field in poloidal and toroidal vectors (Chandrasekhar, 1961, Appendix III) and the temperature field in spherical 
harmonics. We retain only the lowest axisymmetric modes of $\mathbf{U}$ and $T$, that are furthermore symmetric about the equator.

For the angular velocity we obtain (Chandrasekhar, Appendix III, Equation (15))

$$
\begin{aligned}
U_{\phi} & =-\frac{t_{1}(r)}{r} \frac{\partial Y_{1}^{0}}{\partial \theta}-\frac{t_{3}(r)}{r} \frac{\partial Y_{3}^{0}}{\partial \theta} \\
& =\frac{r \sin \theta}{2}\left(\frac{3}{\pi}\right)^{\frac{1}{2}}\left[T_{1}(r)+\frac{(21)^{\frac{1}{2}}}{2} T_{3}(r)\left(5 \cos ^{2} \theta-1\right)\right]
\end{aligned}
$$

Above, $T_{1}(r)\left(=t_{1}(r) / r^{2}\right)$ and $T_{3}(r)\left(=t_{3}(r) / r^{2}\right)$ are some functions of $r ; \theta$ is the polar angle; and the spherical harmonics have been normalized according to Condon and Shortley (1951)

$$
\left(Y_{L}^{m}(\mathrm{CS})=\frac{(-1)^{m}}{\sqrt{2 \pi}}\left[\frac{2 L+1}{2} \frac{(L-m) !}{(L+m) !}\right]^{1 / 2} Y_{L}^{m}(\text { Chandrasekhar })\right)
$$

The meridional motions (i.e., the poloidal components of the axisymmetric velocity field) are also determined by two scalars, $p_{2}(r)$ and $p_{4}(r)$ (stream functions), which define motions with one and two cells, respectively, in each hemisphere $(0<\theta<\pi / 2$ and $\pi / 2<\theta<\pi$ for the northern and southern hemisphere, respectively) of the convection zone.

(Explicitly,

$$
U_{r}=\sum_{L=2,4} \frac{L(L+1)}{r^{2}} p_{L} Y_{L}^{0} ; \quad U_{\theta}=\sum_{L=2.4} \frac{1}{r} \frac{\partial p_{L}}{\partial r} \frac{\partial Y_{L}^{0}}{\partial \theta},
$$

cf. Equation (A3a) of Appendix 1.)

For the temperature we write

$$
T=T_{\mathrm{c}} / \Delta T+(\eta / r-1) /(1-\eta)+\psi(r, t)+\Theta(r, \theta, \phi, t)
$$

In Equation (2.1.3), $\eta=R_{\mathrm{c}} / \boldsymbol{R}_{\mathbf{0}}$; the first two terms are the purely conductive temperature profile, the third term is the distortion of the average temperature due to convection and the fourth term is the fluctuating component of the temperature, which averages to zero on any surface of radius $r$. Note that $\Theta(r, \theta, \phi, t)$ is not only the axisymmetric part of the fluctuating temperature. (It will be clear later that nonaxisymmetric components of the velocity and temperature field play an essential role in the generation of differential rotation and of pole-equator differences in temperature). The lowest axisymmetric mode of $\Theta$ that has symmetry about the equator is

$$
\Theta_{2}(r) Y_{2}^{0}=\frac{1}{4}\left(\frac{5}{\pi}\right)^{\frac{1}{2}} \Theta_{2}(r)\left(3 \cos ^{2} \theta-1\right)
$$

$\Theta_{2}(r)$ defines, therefore, a pole-equator temperature difference.

The main justification for retaining only the lowest terms in the expansions of the axisymmetric modes of $\mathbf{U}$ and $T$ is, of course, mathematical simplicity. (Note, 
however, that the next term in the expansion for the angular velocity is

$$
\frac{T_{5}(r)}{16}\left(\frac{11}{\pi}\right)^{\frac{1}{2}} r \sin \theta\left(315 \cos ^{4} \theta-210 \cos ^{2} \theta+15\right)
$$

which does not have a maximum at the equator.)

In a system of coordinates rotating with an angular velocity $\Omega_{0}$, the equations for $T_{1}(r), T_{3}(r), P_{2}(r)\left(=p_{2}(r) / r\right)$ and $\Theta_{2}(r)$ are found to be (Durney, 1971):

(a) Equations for the angular velocity

$$
\begin{aligned}
\left(\frac{d^{2}}{\mathrm{~d} r^{2}}\right. & \left.+\frac{4}{r} \frac{d}{\mathrm{~d} r}\right) T_{1}+\left(\frac{3}{5}\right)^{\frac{1}{2}} \mathscr{T}_{1}\left(\frac{1}{r} \frac{\mathrm{d} P_{2}}{\mathrm{~d} r}+\frac{3 P_{2}}{r^{2}}\right) \\
& =\frac{(3 \pi)^{\frac{1}{2}}}{4 \sigma r^{4}} \int_{0}^{\pi} \sin ^{2} \theta \frac{\partial}{\partial r}\left(r^{3}\left\langle U_{\phi} U_{r}\right\rangle\right) \mathrm{d} \theta \\
\left(\frac{d^{2}}{\mathrm{~d} r^{2}}\right. & \left.+\frac{4}{r} \frac{d}{\mathrm{~d} r}-\frac{10}{r^{2}}\right) T_{3}+\frac{\mathscr{T}_{1}}{r}\left[\frac{2}{(35)^{\frac{1}{2}}}\left(\frac{\mathrm{d} P_{2}}{\mathrm{~d} r}-\frac{2 P_{2}}{r}\right)\right. \\
& \left.+\frac{5}{(63)^{\frac{1}{2}}}\left(\frac{\mathrm{d} P_{4}}{\mathrm{~d} r}+\frac{5 P_{4}}{r}\right)\right] \\
& =\frac{(7 \pi)^{\frac{1}{2}}}{16 \sigma r} \int_{0}^{\pi} \sin ^{2} \theta\left[\frac{\left(5 \cos ^{2} \theta-1\right)}{r^{3}} \frac{\partial}{\partial r}\left(r^{3}\left\langle U_{\phi} U_{r}\right\rangle\right)\right. \\
& \left.+\frac{10}{r} \cos \theta \sin \theta\left\langle U_{\theta} U_{\phi}\right\rangle\right] \mathrm{d} \theta .
\end{aligned}
$$

(b) Equations for the meridional circulation

$$
\begin{aligned}
\left(\frac{d^{4}}{\mathrm{~d} r^{4}}+\right. & \left.\frac{4}{r} \frac{d^{3}}{\mathrm{~d} r^{3}}-\frac{12}{r^{3}} \frac{d^{2}}{\mathrm{~d} r^{2}}+\frac{24}{r^{4}}\right) P_{2} \\
& -\mathscr{T}_{1}\left[\frac{4}{(35)^{\frac{1}{2}}}\left(5 T_{3}+r \frac{\mathrm{d} T_{3}}{\mathrm{~d} r}\right)+\frac{1}{(15)^{\frac{1}{2}}} r \frac{\mathrm{d} T_{1}}{\mathrm{~d} r}\right]-\mathscr{R}_{1} g_{1}(r) \Theta_{2} \\
= & -\frac{(5 \pi)^{\frac{1}{2}}}{4 \sigma r} \int_{0}^{\pi} \sin \theta\left[\cos \theta \sin \theta \frac{\partial}{\partial r}\left(r\langle\mathbf{U} \cdot \nabla \mathbf{U}\rangle_{\theta}\right)\right. \\
& \left.+\langle\mathbf{U} \cdot \nabla \mathbf{U}\rangle_{r}\left(3 \cos ^{2} \theta-1\right)\right] \mathrm{d} \theta
\end{aligned}
$$

(c) Equations for the pole-equator temperature difference

$$
\begin{aligned}
\left(\frac{d^{2}}{\mathrm{~d} r^{2}}\right. & \left.+\frac{2}{r} \frac{d}{\mathrm{~d} r}-\frac{6}{r^{2}}\right) \Theta_{2}+\frac{6}{r} P_{2}\left(\frac{\eta}{(1-\eta) r^{2}}-\frac{\partial \psi}{\partial r}\right) \\
& =\frac{(5 \pi)^{\frac{1}{2}}}{4 r} \int_{0}^{\pi} \sin \theta\left[\left(3 \cos ^{2} \theta-1\right) \frac{1}{r} \frac{\partial}{\partial r}\left(r^{2}\left\langle U_{r} \Theta\right\rangle\right)\right. \\
& \left.+6\left\langle U_{\theta} \Theta\right\rangle \cos \theta \sin \theta\right] \mathrm{d} \theta .
\end{aligned}
$$


where $g_{1}(r)=g(r) / r ; \quad \mathscr{T}_{1}=2 \Omega_{0} R_{0}^{2} / \nu \quad\left(=\mathscr{T}^{1 / 2} /(1-\eta)^{2}\right) ; \quad \mathscr{R}_{1}=\alpha \Delta T g\left(R_{0}\right) R_{0}^{3} / \kappa \nu$ $\left(=\mathscr{R} /(1-\eta)^{3}\right) ; P_{4}(r)=p_{4}(r) / r ; \sigma(=\nu / \kappa)$ is the turbulent Prandtl number and $\langle A B\rangle$ is defined by $\langle A B\rangle=(1 / \pi) \int_{0}^{2 \pi} A B \mathrm{~d} \phi$. We chose $R_{0}$ and not the thickness of the convective shell as the unit of distance. This is the reason why $\mathscr{T}_{1}$ is not the square root of the Taylor number $(\mathscr{T})$; also the usual Rayleigh number is $\mathscr{R}$ and not $\mathscr{R}_{1}$.

Turbulent convection is assumed to determine $\nu$ and $\kappa$ in Equations (2.1.5)(2.1.8); $\mathbf{U}$ and $\Theta$ are the large-scale velocity and temperature. Following Unno (1961), it will be assumed that $\sigma \sim 1$. The value of the turbulent viscosity, $\nu$, does not change much in the convection zone and at a depth of $1.8 \times 10^{10} \mathrm{~cm}, \nu \sim$ $8 \times 10^{12} \mathrm{~cm}^{2} \mathrm{~s}^{-1}$ (see Table I in Section 2.2). The value of $\mathscr{T}_{1}$ is known; the value of $\mathscr{R}_{1}$ which determines the strength of the large-scale convection, is not known. In these theories a natural way of determining $\mathscr{R}_{1}$ is by requiring that the value of $T_{3}\left(R_{0}\right)$ (which determines the latitudinal differential rotation at the surface by Equation (2.1.2) should agree with the observed value. The thickness of the convective layer is not well-known either: one can assume that it is a scale height or the actual depth of the solar convection zone.

The boundary conditions corresponding to specified temperatures and zero radial velocities and stresses at $r=R_{\mathrm{c}}, R_{0}$ are $\Theta_{2 L}=P_{2 L}=P_{2 L}^{\prime \prime}=T_{2 L+1}^{\prime}=0$ at $r=\eta, 1$ $(L=1,2) ; T_{1}^{\prime}=0$ at $r=\eta$; and $\int_{\eta}^{1} r^{4} T_{1}(r) \mathrm{d} r=0$. (If $T_{1}^{\prime}=0$ at $r=\eta$, then in the steady state also $T_{1}^{\prime}=0$ at $r=1 ; \int_{\eta}^{1} r^{4} T_{1}(r) \mathrm{d} r=0$ implies that, in the rotating system of coordinates, the total angular momentum of the spherical shell is zero.)

The only approximation used in deriving Equations (2.1.5)-(2.1.8) is the Boussinesq approximation. In other words, suppose that we have a convective, rotating spherical layer of fluid and that $P_{4}(r)$ is negligible (or known), then if we could measure $\psi(r)$ and the azimuthal averages of the fluctuating quantities appearing in the right-hand side of Equations (2.1.5)-(2.1.8), these equations would allow us to determine $T_{1}(r), T_{3}(r), P_{2}(r)$, and $\Theta_{2}(r)$. In the right-hand side of Equations (2.1.5)-(2.1.8), $\Theta$ has been defined by Equation (2.1.3) and $\mathbf{U}$ is the total velocity field; $\mathbf{U}$ and $\Theta$ contain, therefore, non-axisymmetric as well as axisymmetric components, and it is apparent that the solution of Equations (2.1.5)-(2.1.8) poses a formidable problem. An approximate method of solution that clearly shows how $T_{1}$, $T_{3}, P_{2}$ and $\Theta_{2}$ are generated is the following:

(i) The problem of a rotating, convective spherical layer of fluid is first solved in the quasi-linear approximation (Herring, 1963, 1964, 1969; Durney, 1968a). In this approximation, which has been shown to be qualitatively successful, the fluctuating self-interactions (i.e., the terms $\mathbf{U} \cdot \nabla \mathbf{U}, \mathbf{U} \cdot \nabla \Theta$ ) are neglected; the only non-linear term that is retained is the product of the distortion of the mean temperature and the velocity $\left[U_{r}(\partial \psi / \partial r)\right]$ (the equations for the different modes of the temperature and velocity field in this approximation are given in Appendix 1). The solution of this problem shows that convection in the presence of rotation is highly nonaxisymmetric.

(ii) With the values of $U$ and $\Theta$ evaluated in (i) we can calculate the right-hand side of Equations (2.1.5)-(2.1.8) (RHS (2.1.5-2.1.8)), and solve for $T_{1}, T_{3}, P_{2}$ and $\Theta_{2}$ (for $\psi$, we use the value found in (i)). It is now clear how the large-scale convection in the presence of rotation generates, through the products of the fluctuating quantities appearing in the RHS (2.1.5-2.1.8), the axisymmetric modes of the temperature and 
velocity field. In Appendix II we give an order-of-magnitude estimate, based on dimensional considerations, of the RHS (2.1.5-2.1.8) and of $T_{3}$. In the method of solution described above, we have neglected the effect of the axisymmetric modes on the large-scale non-axisymmetric convection and on $\psi$. Gilman (1972) has taken these effects into account in the framework of the mean field approximation.

If $U_{r}=P_{2}=T_{1}^{\prime}=0$ at $r=\eta$, the integration of Equation (2.1.5) gives

$$
r^{4} \frac{\mathrm{d} T_{1}}{\mathrm{~d} r}+\left(\frac{3}{5}\right)^{\frac{1}{2}} \mathscr{T}_{1} r^{3} P_{2}=\frac{(3 \pi)^{\frac{1}{2}}}{4 \sigma} \int_{0}^{\pi} \sin ^{3} \theta\left\langle U_{\phi} U_{r}\right\rangle \mathrm{d} \theta
$$

which expresses that the angular momentum transport across a spherical surface due to (a) viscosity and (b) the action of Coriolis forces on the meridional circulation, is equal (in the steady state) to the angular momentum transport due to the Reynold's stresses. Equations (2.1.5) and (2.1.9) are equations for the radial differential rotation.

Equation (2.1.6) shows how the vertical $\left[(\partial / \partial r)\left(r^{3}\left\langle U_{\phi} U_{r}\right\rangle\right)\right]$ and latitudinal $\left(\left\langle U_{\theta} U_{\phi}\right\rangle\right)$ transports of angular momentum generate the latitudinal differential rotation. The action of Coriolis forces on a meridional circulation can, just by itself, give rise to differential rotation, as is shown by the term in $\mathscr{T}_{1}$ in Equation (2.1.6).

A pole-equator temperature difference can be generated (a) by a vertical variation in the radial heat flux, the term $\left[(\partial / \partial r)\left(r^{2}\left\langle U_{r} \Theta\right\rangle\right)\right]$ in Equation $(2.1 .8)$, (b) by a latitudinal heat flux $\left(\left\langle U_{\theta} \Theta\right\rangle\right)$, or (c) by a meridional circulation, if the average temperature varies with $r$ (the term in $P_{2}$ in the left-hand side of Equation (2.1.8)).

A serious problem plaguing this theory of differential rotation from the beginning has been the large (and unobserved) pole-equator difference in flux $(\Delta \mathscr{F})$ needed to generate the observed solar differential rotation (cf. Figure 4 and the discussion in Durney, 1970 and Gilman, 1972). The basic reason for this difficulty is the following: to generate the observed differential rotation, a sizeable fraction of the energy flux must be transported by the large-scale convection (Equation (B5) of Appendix II). Transport of angular momentum towards the equator arises when the convective motions are mainly longitudinal (i.e. when the longitudinal velocities are large) since in this case the Coriolis forces transport the fast-rotating fluid particles towards the equator and the slow-rotating ones towards the poles (see Yoshimura, 1972a and Appendix II). If the convective motions have large longitudinal components, then something is known about the shape of the convectivecells: $m$ has to be large (see Equation (A3) of Appendix I). If $m$ is large, the large-scale convective motions are peaked at the equator and significant pole-equator differences in flux appear unavoidable (if $m=L$, the $\theta$ dependence of $U_{r}$ is of the form $\sin ^{L} \theta$; if $m \neq L$, the rate of angular momentum transport is not maximum at the equator, Yoshimura, 1972a, Figure 4). It is unlikely that a mixture of modes can solve the heat flux problem. In Figure 5 we give the convective heat flux at the middle of the convective shell as a function of $\theta$, in the case of axisymmetric convection, for a Rayleigh number of 1500 , a Taylor number of 500 and $\eta=0.8$ (see Figures 1 and 6 of Durney, 1968b). The rotation is fast enough to strongly inhibit convection and the quasi-linear approximation can be trusted qualitatively. The axisymmetric mode, plotted in Figure 5, is essentially identical to the polar modes (with $m=0$ ) plotted in Figure 6 of Gilman 


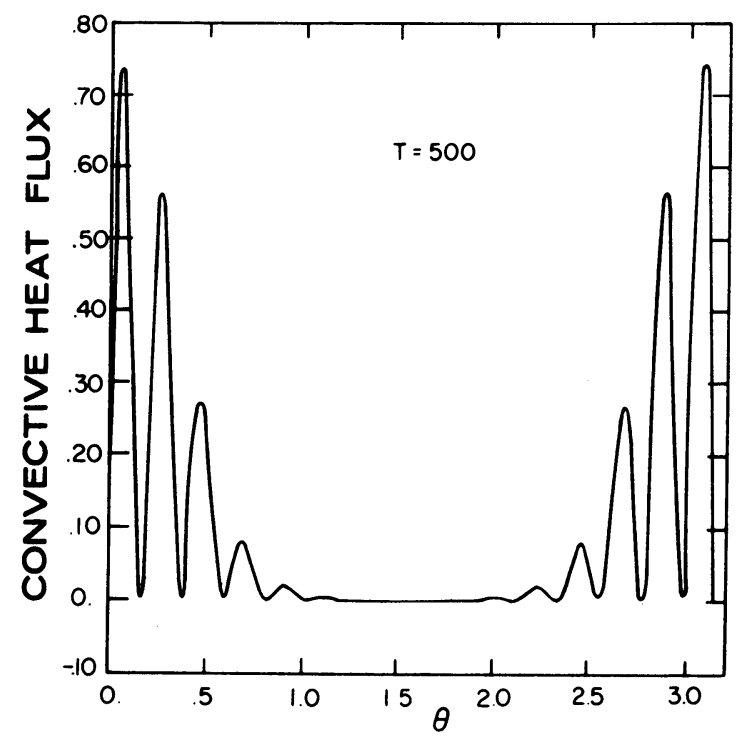

Fig. 5. Convective heat flux versus polar angle for a Rayleigh number of 1500 at the middle layer of a convective shell. Convection is axisymmetric and the rotation rate is fast enough to inhibit convection almost completely.

(1975). Gilman's calculations (for the case of marginal instability and for a variety of Taylor numbers and values of $\boldsymbol{m}$ ) show furthermore that for large Taylor numbers most modes peak at the equator, and that a few modes (with small $m$ 's) peak at the poles, with virtually no modes in between.

The right mixture of modes could give a small enough $\Delta \mathscr{F} ;$ it would, however, in all probability give a wrong profile for the latitudinal differential rotation since the modes that transport angular momentum towards the equator are also those that give rise to a large $\Delta \mathscr{F}$.

It is tempting to assume, therefore, that the main effect of the interaction of rotation with convection is the generation, through the RHS (2.1.8), of a small pole-equator difference in flux; this $\Delta \mathscr{F}$ will drive a meridional circulation $\left(P_{2}\right)$ which, by Equations (2.1.5) and (2.1.6), will generate a radial and latitudinal differential rotation. It is of interest to calculate the magnitude of $\boldsymbol{P}_{2}$ that could explain the observed solar differential rotation. From Equation (2.1.6) we obtain, very crudely, $T_{3} \sim \mathscr{T}_{1} P_{2} /(35)^{\frac{1}{2}}$. Comparing this value of $T_{3}$ with Equation (B3) of Appendix II, we obtain $P_{2}=2 / 15(\pi / 5)^{\frac{1}{2}} \sigma$; if $\sigma=1$ and $\kappa=2 \times 10^{12} \mathrm{~cm}^{2} \mathrm{~s}^{-1}$, we find $U_{r} \sim 6 \mathrm{~cm} \mathrm{~s}^{-1}$.

To generate a latitudinal differential rotation with equatorial acceleration, the meridional ciculation must, however, rise at the poles and sink at the equator. This point, which is treated in detail in Section 2.3, can also be understood as follows: near the outer surface we approximate Equation (2.1.6) to $10 T_{3} / r^{2}=2 \mathscr{T}_{1} P_{2}^{\prime} /(35)^{\frac{1}{2}} r$. For the equator to rotate faster, $T_{3}$ and therefore $P_{2}^{\prime}=\mathrm{d} P_{2} / \mathrm{d} r$ must be negative. Since $P_{2}\left(r=R_{0}\right)=0, P_{2}$ must be positive below the surface and this implies that the motions must sink at the equator. There is no reason to expect this to happen in a 
Boussinesq fluid. In fact, since rotation inhibits convection preferentially at the poles, a circulation rising at the equator (which would increase the equatorial flux) appears more plausible. If differential rotation is generated by a meridional circulation the strong variation of $\rho$ with $r$ must, therefore, be included (Durney, 1972a).

It has been argued here that the angular momentum transport by the Reynold's stresses, in a Boussinesq fluid, cannot explain by itself the observed solar differential rotation. The strong variation of density with depth in the solar convection zone appears to be essential for the understanding, even in a qualitative way, of the solar differential rotation.

This result suggests that differential rotation cannot be generated in the lower part of the convection zone by the interaction of rotation with the global convection. It would be premature to conclude, however, that this is certainly the case, since the action of rotation on the turbulent diffusivity and viscosity has not been taken into account; it is not known what effect this action could have on theories of the thermodynamics and dynamics of the lower part of the convection zone.

There can be little doubt that global convection exists in the Sun: the observations (in particular 1.3 and the existence of giant magnetic field structures seem to corroborate it (cf. also Howard and Yoshimura's (1976) paper in these proceedings) Global convection could, however, be very nearly critical and therefore transport little energy flux. In this context the existence of periods in the past when the solar cycle appeared to have died off could be of importance; it has been suggested by Yoshimura (1976a) that during these periods global convection was very weak and could even have ceased to exist. It is easy to imagine this happening if global convection were very nearly critical. (In Yoshimura's model of the solar cycle, global convection regenerates the poloidal magnetic field from the toroidal field.)

Rigid rotation appears in this theory of differential rotation in a natural way because of the existence of waves. Waves of the velocity field have been discussed in Appendix I. Yoshimura $(1971,1972 a, b)$ has shown that Švestka's observations pertaining to the rigid rotation of complexes of activity (cf. Section 1.3) can be explained, in terms of the time dependence of the magnetic field, as resulting from its interaction with rotation and the global convection.

\subsection{NON-BOUSSINESQ THEORIES}

The study of convection in a compressible medium (cf. Skumanich, 1955; Böhm, 1963; Spiegel, 1964, 1965; Vickers, 1971; Heard, 1973; Vandakurov, 1975a, b) is a difficult subject even in the absence of rotation; it is not surprising, therefore, that non-Boussinesq theories of differential rotation (Vandakurov, 1975b) are in a more primitive state of development than Boussinesq theories. This subject could, however, be basic for an understanding of the solar differential rotation and a brief discussion is called for here.

The convection zone is strongly stratified with depth. In Table I (cf. Table 1 of Cocke, 1967, and Baker and Temesvary, 1966) the values of the following quantities are tabulated as a function of depth: the density $(\rho)$; the temporature $(T)$; the mixing length $(l)$, chosen equal to $1.5 \times$ pressure scale height; the turbulent convective 
velocity $\left(u_{c}\right)$; the dynamic turbulent viscosity $\left(\eta=\rho u_{c} l / 3\right)$; the kinematic turbulent viscosity $\left(\nu=u_{c} l / 3\right) ;|\mathrm{d} \eta / \mathrm{d} r| / \rho$; the ratio of the convective flux to the total energy flux $\left(F_{C} / F_{T}\right)$; the superadiabatic gradient $(\nabla \Delta T)$; the square root of the local turbulent Taylor number $\left(\mathscr{T}^{\frac{1}{2}}=2 \Omega_{0} l^{2} / \nu=6 \Omega_{0} l / u_{c}\right.$; more appropriately, therefore, $\mathscr{T}^{\frac{1}{2}}$ is proportional to the inverse of the Rossby number), and the turbulent Rayleigh number $\left(\mathscr{R}=\alpha g \nabla \Delta T d^{4} / \kappa\right)$. An approximate expression for the superadiabatic gradient was evaluated from the mixing length expression for the convective flux:

$$
\mathscr{F}=\frac{l}{2} c_{p} \rho u_{c} \nabla \Delta T ; \quad \nabla \Delta T=T\left[\left(1-\frac{1}{\gamma}\right) \frac{\nabla p}{p}-\frac{\nabla T}{T}\right]
$$

with $\mathscr{F}=\left(R_{0} / r\right)^{2} \mathscr{F}_{0}\left(F_{C} / F_{T}\right), \mathscr{F}_{0}$ being the energy flux at the surface; $c_{p}=$ $\gamma R_{g} / \mu(\gamma-1)$ with $\gamma=5 / 3, R_{g}$ the gas constant, and $\mu$ the molecular weight $(=0.6)$. In the Rayleigh number $\alpha=1 / T, d\left(=1.9 \times 10^{10} \mathrm{~cm}\right)$ is the depth of the convection zone and $\kappa=\nu$ (the turbulent Prandtl number is equal to one).

The problem of global convection in a compressible medium (cf. Vickers, 1971; Heard, 1973; Vandakurov, 1975a, b) can be clarified with the help of the following simple and crude model: since the kinematic turbulent viscosity, $\nu$, does not change much in the convection zone, Equation (2.2.1) gives

$$
\nabla \Delta T=\alpha / \rho ; \quad \alpha=2 \mathscr{F} / 3 c_{p} \nu
$$

wherein the first approximation $\alpha$ can be taken as a constant. The equation of hydrostatic equilibrium, the gas equation and Equation (2.2.2) determine the unperturbed state. If motions are present, the relevant equations could be taken as the Navier-Stokes equations with a kinematic viscosity $\nu$, the usual continuity and gas equations and the following energy equation

$$
\operatorname{div} \mathscr{F}=c_{p} \rho \mathbf{U} \cdot \nabla \Delta T
$$

where $\mathbf{U}$ is the large-scale velocity and $\mathscr{F}$ is given by Equation (2.2.1).

The problem of marginal stability (Vickers, 1971; Heard, 1973) consists, then, in determining the value of $\alpha\left(\alpha_{c}\right)$ such that the system is stable for $\alpha<\alpha_{c}$ and unstable for $\alpha>\alpha_{c}$. Vickers' (1971) calculations (performed, however, with a different energy equation, which in the unperturbed state is $\kappa \mathrm{d} T / \mathrm{d} z=$ constant) lend support to the idea that the value of $\alpha$, as calculated from Baker and Temesvary's tables, for example, is larger than $\alpha_{c}$. In other words, the solar convection zone is unstable against global convection. Vickers and Heard (who also included rotation) furthermore find that the large-scale convection is concentrated in the lower part of the convection zone (i.e., the preferred modes are damped in the upper portions of the layer). This result is somewhat surprising since the Rayleigh number is large in the upper part of the convection zone (cf. Table I); could this result be due to the boundary condition $U_{r}=0$ at $r=R_{0}$ ? In the Sun the radial velocities do not vanish at the surface but can overshoot into the stable photospheric layers. Whatever the case, it is important to establish firmly (with respect to both the interpretation of the observations and to the generation of the solar differential rotation) whether the global convection is indeed concentrated in the lower part of the convection zone. If this were the case the Boussinesq theories should be qualitatively correct (see, however, the last paragraphs of this section) and, therefore, we would again expect that (i) a relation should 
B. R. DURNEY

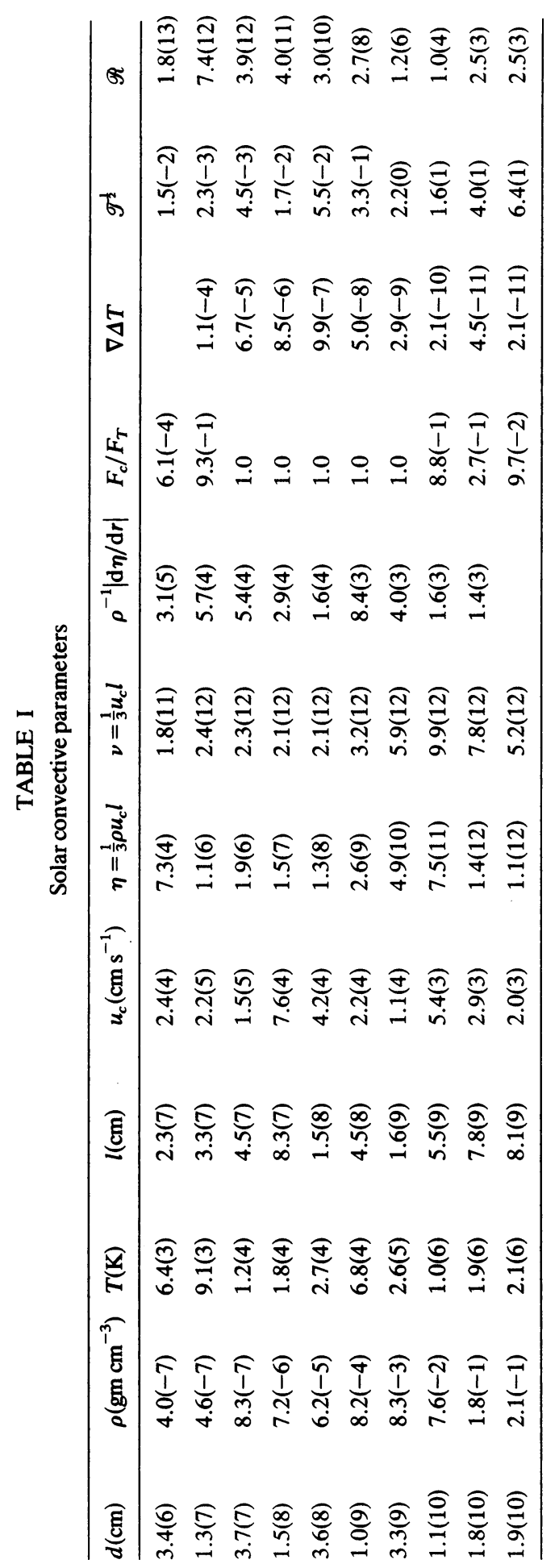


exist between the angular momentum transport by the Reynolds stresses and the energy carried by the large-scale convection, and (ii) other processes than this angular momentum transport should be of importance in the generation of the solar differential rotation.

If, on the other hand, the velocities of the global convection are large only in the surface layers, then point (i) above ceases to be valid: the large-scale convection could carry small amounts of energy and at the same time the large surface azimuthal velocities could give rise to a significant transport of angular momentum towards the equator. If the Sun's differential rotation is generated in the upper part of the convection zone by angular momentum transport, then differential rotation should be 'large,' i.e., we would expect the Reynolds number of the azimuthal flow to be of the order of the critical Reynolds number. Let $\Delta \Omega$ be the difference in angular velocity between the equator and the poles. On a sphere of radius $R_{0}$, differential rotation gives rise to a shear flow with a Reynolds number given by

$$
\mathscr{R}_{e}=\Delta \Omega R_{0}^{2} / \nu
$$

where $\nu$ is the turbulent viscosity. The turbulent viscosity associated with the granules is easy to evaluate; we take $u \sim 0.4 \mathrm{~km} \mathrm{~s}^{-1}$ (cf. Beckers and Morrison, 1970; Mehltretter, 1971; Mattig and Nesis, 1974) and $l \sim 10^{3} \mathrm{~km}$. Therefore, $\nu \sim u l \sim 4 \times$ $10^{12} \mathrm{~cm}^{2} \mathrm{~s}^{-1}$, and $\mathscr{R}_{e} \sim 9 \times 10^{2}$. The Reynolds number evaluated with the turbulent viscosity due to the supergranules appears to be somewhat smaller. The supergranular velocities decrease sharply with depth (Appenzeller and Schröter, 1968). We take $u \sim 0.14 \mathrm{~km} \mathrm{~s}^{-1}$ and $l \sim 1.5 \times 10^{4} \mathrm{~km}$, and find $\mathscr{R}_{e} \sim 1.7 \times 10^{2}$. These values of the Reynolds number should be compared with the critical Reynolds number, which is about $10^{3}$. An important question that can be raised at this point is whether differential rotation penetrates deeply into the convection zone in the case when it is generated in the surface layers.

If the convection zone is highly unstable against large-scale convection $\left(\alpha \gg \alpha_{c}\right)$ then a more reliable method of calculating the dominant modes is to calculate growth rates (Vandakurov, 1975a); a model of the convection zone is assumed to be given (in our example, the value of $\alpha$ ) and the growth rates of different large-scale perturbations are calculated. Vandakurov (1975a) takes $\nabla \Delta T$ proportional to $T$ and assumes that $\Omega_{0} T^{\frac{1}{2}} /(\nabla \Delta T)^{\frac{1}{2}} g^{\frac{1}{2}} \ll 1$ where $g$ is gravity; this inequality is valid in the upper portions of the convection zone (see Table I). The dominant mode is assumed to be the one with the largest growth rate. Vandakurov (1975b) has generalized his method to include (in a crude way) nonlinear terms and finds that in general the upper parts of stellar convection zones should rotate differentially, with no appreciable variations in the heat flux with latitude. However, large variations of $\mathscr{F}$ with $\theta$ are expected only in the lower part of the convection zone (see 2.4) and this problem cannot be considered solved at present. There can be no doubt that in order to fully explain the solar differential rotation, we need a much deeper understanding of the large-scale convection in a compressible model of the solar convection zone.

We have used Equations (2.2.3) and (2.2.1) as the energy equation for a compressible fluid and Equation (A1b) of Appendix 1 as the Boussinesq energy equation. It is important to understand under which approximations Equations (2.2.3) and (2.2.1) 
reduce to Equation (A1b). Equation (2.2.3) can also be written in the following form

$$
\operatorname{div} \mathscr{F}=\mathbf{U} \cdot\left[\nabla p-c_{p} \rho \nabla T\right] .
$$

With the help of the hydrostatic relation the right hand side of Equation (2.2.5) becomes $-\rho c_{p} \mathbf{U}\left[\nabla T+g / c_{p}\right]$. The expression for the convective flux can be written $\mathscr{F}=-(l / 2) \rho u_{c} c_{p}\left[\nabla T+g / c_{p}\right]$. Therefore, if the variations of $\rho c_{p}$ can be neglected we obtain

$$
\operatorname{div} \mathscr{F}+\mathbf{U}\left(\nabla T+g / c_{p}\right) ; \quad \mathscr{F}=-\frac{l u_{c}}{2}\left(\nabla T+g / c_{p}\right)
$$

Apart from the factor $g / c_{p}$ (the adiabatic gradient) Equation (2.2.6) is the usual energy equation for an incompressible fluid (Equation (A1b) of Appendix 1). The thermometric diffusivity is equal to $l u_{c} / 2$ (which is approximately constant in the Sun) and the Prandtl number is close to one, $(\sigma=\nu / \kappa=2 / 3)$.

\subsection{Meridional Circulation}

The idea that the Sun's differential rotation could be generated by a meridional circulation driven by a pole-equator temperature difference (due to the interaction of rotation with convection) was first put forward by Weiss (1965) and Veronis (1966) (see also Osaki, 1970; Roxburgh, 1970; Durney and Roxburgh, 1971; Durney, 1974; and Gierasch, 1974).

We will neglect the Reynolds stresses due to the large-scale convection and approximate the action of the turbulent convection by a turbulent viscosity (assumed furthermore to act as a molecular viscosity). We can limit ourselves to axially symmetric flows since it is clear that any pole-equator difference in temperature generated by the interaction of rotation with convection will be axially symmetric.

The time-dependent Navier-Stokes equations can then be written

$$
\begin{aligned}
& \rho\left[\frac{D U_{r}}{D t}-\frac{U_{\theta}^{2}+U_{\phi}^{2}}{r}\right]=-\frac{\partial p}{\partial r}-\rho g+R_{r} \\
& \rho\left[\frac{D U_{\theta}}{D t}+\frac{U_{r} U_{\theta}}{r}-\frac{U_{\phi}^{2} \operatorname{cotg} \theta}{r}\right]=-\frac{1}{r} \frac{\partial p}{\partial \theta}+R_{\theta} \\
& \rho\left[\frac{D U_{\phi}}{D t}+\frac{U_{\phi} U_{r}}{r}+\frac{U_{\theta} U_{\phi} \operatorname{cotg} \theta}{r}\right]=R_{\phi}
\end{aligned}
$$

where $D / D t=U_{r} \partial / \partial r+\left(U_{\theta} / r\right) \partial / \partial \theta$ and $R_{r}, R_{\theta}, R_{\phi}$ are the components of the viscous force written down explicitly in Appendix III.

It is readily seen that if $\gamma$ (the ratio of the specific heats) and $c_{v}$ are constants, then the time-independent energy equation

$$
\rho \mathbf{U} \cdot \nabla c_{v} T-\frac{p}{\rho} \mathbf{U} \cdot \nabla \rho+\operatorname{div} \mathscr{F}=0
$$

can also be written as in Equation (2.2.3); 70 and $\nabla T$ are defined in Equation (2.2.1). Equation (2.2.1) is the expression for the turbulent convective flux only; no allowance is made for a possible contribution from the large-scale convection. The 
reason is simple: not enough is known about the large-scale compressible convection. In the development of these theories, it is therefore necessary to assume that rotation acts as a perturbation on the turbulent convective flux.

An illustrative example that clarifies the basic ideas behind these theories of differential rotation is the following (cf. Belvedere and Paterno, 1976): consider a rotating spherical layer of fluid with a thermal conductivity of the form $\kappa=$ $\kappa_{0}\left[1+\xi(r) P_{2}(\cos \theta)\right]$. The factor in the bracket mimics the effect of rotation on convection, which depends on latitude and depth. Even if the fluid is not convective, meridional motions will be set up and the action of Coriolis forces on these motions will generate differential rotation. The replacement of 'thermal conduction' by 'turbulent convection' gives us a clear picture of the theories of differential rotation discussed in this section. The fluid, however, could also be convective, which would correspond to the large-scale convection. The action of rotation on these large-scale convective motions is not taken into account. This effect could be important; in particular, the dependence on depth of the interaction of rotation with (i) the large-scale convection and (ii) the turbulent convection could be different.

The action of rotation on turbulent convection can be introduced by replacing Equation (2.2.1) with Equation (2.3.5), for example:

$$
\mathscr{F}=\left[1+\varepsilon \frac{2 \Omega_{0} l^{2}}{\nu} P_{2}(\cos \theta)\right] \frac{l}{2} u_{c} c_{p} \rho \nabla \Delta T
$$

(a version of this equation was used in Durney and Roxburgh, 1971). The interaction of rotation with convection depends on latitude (since the angle between the angular velocity and gravity depends on $\theta$ ) and on depth (since the scale of the convective motions is a strong function of $r$ ). This interaction is contained in the factor $\varepsilon 2 \Omega_{0} l^{2} / \nu$ of Equation (2.3.5) and has, therefore, been assumed to be proportional to the square root of the Taylor number or the inverse of the Rossby number (see Table I). This appears to be a reasonable choice since in the Boussinesq theories the effect of rotation manifests itself through $\mathscr{T}^{\frac{1}{2}}$. The proportionality factor $\varepsilon$ can be fixed by requiring that the calculated and observed values of the latitudinal differential rotation at the Sun's surface are in agreement.

An understanding of these calculations, and of the relation between the meridional circulation and differential rotation, is particularly simple in the limit of large viscosity and slow rotation. In this case, Equations (2.3.1) and (2.3.2) simplify to

$$
-\frac{\partial p}{\partial r}-\rho g+R_{r}=0 ; \quad-\frac{1}{r} \frac{\partial p}{\partial \theta}+R_{\theta}=0 .
$$

The viscous terms $R_{r}$ and $R_{\theta}$ depend only on $U_{r}$ and $U_{\theta}$ (see Appendix III); $U_{r}$ and $U_{\theta}$ are defined in terms of the stream function, $\psi_{1}(r)$, by

$$
U_{r}=\frac{\partial \psi_{1}}{\partial \theta} / \rho r^{2} \sin \theta ; \quad U_{\theta}=-\frac{\partial \psi_{1}}{\partial r} / \rho r \sin \theta
$$

Equations (2.3.6) allow us to express $p$ and $\rho$ (and therefore also $T$, by the gas equation) in terms of $\psi_{1}$. The energy equation (Equation 2.2.3) is then an equation for $\psi_{1}$. Once $\psi_{1}$ is known, the azimuthal equation of motion determines the angular velocity. An appropriate value of $\varepsilon$ would then reproduce the observed differential 
rotation at the Sun's surface. A method of solution could be the following: we first expand all quantities in terms of Legendre polynomials:

$$
\begin{aligned}
& p=p_{u}\left(1+p_{2}(r) P_{2}(\cos \theta)\right) ; \quad \rho=\rho_{u}\left(1+\rho_{2}(r) P_{2}(\cos \theta)\right) \\
& T=T_{u}\left(1+T_{2}(r) P_{2}(\cos \theta)\right) \\
& U_{r}=\frac{2 \psi(r)}{\rho r^{2}} P_{2}(\cos \theta) ; \quad U_{\theta}=-\frac{\partial \psi}{\partial r} \sin \theta \cos \theta / \rho r .
\end{aligned}
$$

The subscript $u$ denotes the unperturbed non-rotating state and we have chosen a particular form for the stream function $\psi_{1}\left(=\sin ^{2} \theta \cos \theta \psi(r)\right)$. Equations (2.3.9) describe motions with one latitudinal cell in each hemisphere. Appendix IV contains the expressions for $p_{2}$ and $\rho_{2}$ in terms of $\psi$.

With $p, \rho$, and $T$ given by Equation (2.3.8), the expression for the superadiabatic gradient becomes

$$
\begin{aligned}
\nabla \Delta T= & (\nabla \Delta T)_{u} \hat{i}_{r}+\left\{T_{2}(\nabla \Delta T)_{u}+T_{u}\left[(1-1 / \gamma) p_{2}^{\prime}-T_{2}^{\prime}\right]\right\} P_{2}(\cos \theta) \hat{i}_{r} \\
& -3 \frac{\sin \theta \cos \theta}{r} T_{u}\left[(1-1 / \gamma) p_{2}-T_{2}\right] \hat{i}_{\theta}
\end{aligned}
$$

where $\hat{i}_{r}$ and $\hat{i}_{\theta}$ are unit vectors in the $r$ and $\theta$ directions, respectively. We write Equation (2.3.10) in the condensed form

$$
\nabla \Delta T=(\nabla \Delta T)_{u} \hat{i}_{r}+\delta(\nabla \Delta T),
$$

where the definition of $\delta(\nabla \Delta T)$ follows immediately from Equation (2.3.10). To the first order in the perturbed quantities the energy equation (Equation 2.2.3) can be written (we use Equations (2.2.1), (2.3.9), and (2.3.10) for $\mathscr{F}, \mathbf{U}$, and $\nabla \Delta T$, respectively)

$$
\begin{gathered}
\operatorname{div}\left(\frac{l}{2} u_{c} c_{p} \rho_{u} \delta(\nabla \Delta T)\right)-2 \psi(r) c_{p} P_{2}(\cos \theta)(\nabla \Delta T)_{u} / r^{2} \\
=-\frac{2 \varepsilon \Omega_{0}}{\nu r^{2}} P_{2}(\cos \theta) \mathscr{F}_{0} R_{0}^{2} \frac{\partial l^{2}}{\partial r}
\end{gathered}
$$

where $\mathscr{F}_{0}$ is the solar energy flux $\left(\mathrm{erg} \mathrm{cm}^{-2}\right)$ at the surface. We have taken, therefore, a simplified version of the mixing length expression for the convective flux, namely, $\mathscr{F}=l / 2 u_{c} c_{p} \rho_{u} \nabla \Delta T$, with $l u_{c} / 2=\frac{3}{2} \nu$ a constant. It is reasonable to expect that the scale of variation of $T_{u}, p_{2}$, and $T_{2}$ is smaller than $r$, that is, $|\partial A / \partial r|>A / r$ where $A$ stands for $T_{u}, p_{2}$, and $T_{2}$. We shall therefore neglect the term containing the $\theta$-derivative in $\operatorname{div}\left[(l / 2) u_{c} c_{p} \rho_{u} \delta(\nabla \Delta T)\right]$. Furthermore, since $(\nabla \Delta T)_{u}$ is small we neglect $T_{2}(\nabla \Delta T)_{u}$ with respect to $T_{u}\left[(1-1 / \gamma) p_{2}^{\prime}-T_{2}^{\prime}\right]$ in $\delta \nabla \Delta T$. Equation (2.3.11) then reduces to

$$
\frac{d}{\mathrm{~d} r}\left[r^{2} \frac{l}{2} \frac{u_{c}}{\gamma-1} p_{u}\left((\gamma-1) p_{2}^{\prime}-\gamma T_{2}^{\prime}\right)\right]-2 \psi c_{p}(\nabla \Delta T)_{u}=-\frac{2 \varepsilon \Omega_{0}}{\nu} \mathscr{F}_{0} R_{0}^{2} \frac{\partial l^{2}}{\partial r}
$$


The ratio $(Q)$ of the second term to the first term in the left-hand side of Equation (2.3.12) is of the order $Q \sim \mathscr{F}_{0} / \rho\left(l_{\psi} / \nu\right)^{3}(l / r)^{2}$ where $l_{\psi}^{3}=\psi / \psi^{\prime \prime \prime}$. In the estimate of $Q$, Equation (2.2.2) was used for $(\nabla \Delta T)_{u}^{-}$, and $\left(p_{u} p_{2}^{\prime}\right)^{\prime}$ was evaluated with the help of Equation (D2) $\left(\left(p_{u} p_{2}^{\prime}\right)^{\prime} \sim \nu \psi^{\prime \prime \prime}\left(\rho^{\prime} / \rho\right)\left(p^{\prime} / p\right)\right)$. It is clear that if $\nu$ is sufficiently large $\left(>\sim 2 \times 10^{13} \mathrm{~cm}^{2} \mathrm{~s}^{-1}\right)$, the term $2 \psi c_{p}(\nabla \Delta T)_{u}$ in Equation (2.3.12) can be neglected. Furthermore, if we set $r=R_{0}$ in the first term of Equation (2.3.12) we obtain

$$
(\gamma-1) p_{2}^{\prime}-\gamma T_{2}^{\prime}=-\frac{4 \Omega_{0}(\gamma-1) \varepsilon}{3 \nu^{2}} \mathscr{F}_{0} l^{2} / p_{u}
$$

The integration constant was chosen equal to zero for the following reasons: by virtue of Equations (2.3.5) and (2.3.10), the pole-equator difference in flux is given by $\mathscr{F}_{0}\left[\gamma T_{2}+T_{u}\left[(\gamma-1) p_{2}^{\prime}-\gamma T_{2}^{\prime}\right] /(\nabla \Delta T)_{u}+2 \varepsilon \Omega_{0} l^{2} \gamma / \nu\right] P_{2}(\cos \theta) / \gamma$, which reduces to $\mathscr{F}_{0} T_{2} P_{2}(\cos \theta)$ (a small quantity) if Equation (2.3.13) is satisfied. (Since $\nu=l u_{c} / 3$ and $\mathscr{F}_{0}$ is given by Equation (2.2.1) the right-hand side of Equation (2.3.13) can also be written $\left.-2 \Omega_{0} \varepsilon l^{2}(\nabla \Delta T)_{u} \gamma / \nu T_{u}\right)$. Equation (2.3.13) will be solved with the boundary condition $(\gamma-1) p_{2}-\gamma T_{2}=0$ at $r=R_{0}$. Perhaps a better boundary condition would have been $T_{2}=0$ at $r=R_{0}$. It is unlikely, however, that these solutions would differ much. (It would be much better, of course, to impose that the latitudinal variations of the energy flux and temperature vanish at $r=R_{c}$, the lower boundary of the convection zone; the calculations determine then $\Delta \mathscr{F}$ and $T_{2}$ at $r=R_{0}$ ). With the help of Equations (D1) and (D2) of Appendix IV, we can substitute $p_{2}$ and $T_{2}$ in the integrated version of Equation (2.3.13) and obtain explicitly the equation for $\psi$. This is Equation (D3) of Appendix IV. Equation (D3) will be solved by assuming that the unperturbed state satisfies a polytropic relation: $p_{u}=p_{u c}\left(\rho_{u} / \rho_{u c}\right)^{\gamma}$; the pressure, density, and temperature are then given by

$$
\begin{aligned}
& p_{u}=p_{u c}\left(1-\left(r-R_{c}\right) / L\right)^{\gamma /(\gamma-1)} ; \quad \rho_{u}=\rho_{u c}\left(1-\left(r-R_{c}\right) / L\right)^{1 /(\gamma-1)} \\
& T_{u}=T_{u c}\left(1-\left(r-R_{c}\right) / L\right)
\end{aligned}
$$

with

$$
L=\gamma R_{g} T_{c} /(\gamma-1) \mu g
$$

The subscript $c$ denotes quantities at the bottom of the convection zone, as given for example by Baker and Temesvary's tables. We take $l=1.5 p_{u} / p_{u}^{\prime}$; the integral in the right-hand side of Equation (D3) can then be evaluated in closed form (see Equation (D4) of Appendix IV). Appropriate boundary conditions for Equation (D3) (with the right-hand side given by Equation (D4)) are zero radial velocities and stresses at $r=R_{c}, R_{0}$ :

$$
\psi=0 ; \quad \psi^{\prime \prime}=\psi^{\prime}\left(2 / r+\rho^{\prime} / \rho\right)=0 ; \quad r=R_{c}, R_{0} .
$$

In the large viscosity approximation it is therefore possible to determine $\psi$ (apart from a multiplicative factor, since $\varepsilon$ is not yet known) independently of the angular velocity. To obtain the equation for the angular velocity, we expand $\Omega$ in Legendre polynomials and neglect polynomials of a higher order than two:

$$
\Omega=\Omega_{0}\left(1+\omega_{0}(r)+\omega_{2}(r) P_{2}(\cos \theta)\right) .
$$


This is a good approximation for the observed angular velocity at the surface (cf. Durney, 1976). Howard and Harvey's (1970) observations give

$$
\Omega_{0}=2.57 \times 10^{-6} \mathrm{rad} \mathrm{s}^{-1} ; \quad \omega_{2}\left(R_{0}\right)=-0.189
$$

The equations for $\omega_{0}(r)$ and $\omega_{2}(r)$ are Equations (E1) and (E2) of Appendix V. The solution of the problem at hand is particularly simple if we neglect the nonlinear terms in Equations (E1) and (E2): the equations for $\psi, \omega_{0}(r)$, and $\omega_{2}(r)$ can be solved first with $\varepsilon=1$. Since $\omega_{2}(r)$ is proportional to $\psi$, the value of $\omega_{2}(r)$ at the surface will be -0.189 (which is the observed differential rotation) if $\varepsilon=-0.189 / \omega_{2}\left(R_{0}, \varepsilon=1\right)$. The numerical calculations (with $\gamma=5 / 3, \nu=2 \times 10^{12} \mathrm{~cm}^{2} \mathrm{~s}^{-1}$, and $s=1$ ) give $\varepsilon=$ $-1.4 \times 10^{-6}$. (It should be noted, cf. Equation (D3), that $\psi(r)$ is proportional to $\nu^{-3}$ and therefore $\omega_{2}(r)$ (cf. Equation E2) is proportional to $\nu^{-4}$; had the equations been solved with $\nu=2 \times 10^{13} \mathrm{~cm}^{2} \mathrm{~s}^{-1}$ a much larger value of $\varepsilon$ would have been needed to reproduce the observed differential rotation. With $\nu=2 \times 10^{13} \mathrm{~cm}^{2} \mathrm{~s}^{-1}$ we can justify neglecting the term $2 \psi c_{p}(\nabla \Delta T)_{u}$ in Equation (2.3.12).) If $\varepsilon$ is negative then $2 \varepsilon \Omega_{0} l^{2} P_{2}(\cos \theta) / \nu$ is larger than zero at the equator, and it follows from Equation (2.3.5) that rotation stabilizes the poles more than the equator. Therefore, differential rotation with equatorial acceleration is generated, in this model, if rotation stabilizes turbulent convection preferentially at the poles. This result, of course, should not be taken too seriously and it could well depend on the approximations used in solving Equation (2.3.11) (see, for example, Durney and Roxburgh, 1971). It is important to note that unlike the case of the large-scale convection, we do not know whether the turbulent convection is preferentially stabilized by rotation at the equator or at the poles. Furthermore, as stressed by Iroshnikov (1969), the effect of rotation on the turbulent viscous stress tensor could also be important. Table I shows that at a depth of $1.1 \times 10^{10} \mathrm{~cm}$, a typical time associated with the turbulent convection is $l / u_{c} \sim 10^{6} \mathrm{~s}$, which is not small in relation to $1 / \Omega_{0} \sim 10^{6} / 2.57 \mathrm{~s}^{-1}$. Therefore, the effect of rotation on the turbulent convection, particularly in the lower half of the convection zone, cannot be ignored.

Cowling (1951) has studied the local conditions for instability in polytropic rotating stars. He finds that in the case of uniform rotation, instability will occur if $(\alpha=(1-\gamma / \Gamma) / \gamma H)$

$$
\alpha\left[m^{2}+(l \cos \theta-n \sin \theta)^{2}\right]>4 \Omega_{0}^{2} n^{2} .
$$

Here $\Gamma$ is the polytropic index $\left(p / p_{0}=\left(\rho / \rho_{0}\right)^{\Gamma}\right) ; H$ is the scale height, defined by $H=p / \rho g=p /|\nabla p| ; \theta$ is, as usual, the polar angle and $m, l$, and $n$ are defined by $\partial / \partial R=i l, \partial / R \partial \phi=i m, \partial / \partial z=i n$ (Cowling takes cylindrical polar coordinates $R, \phi$, and $z$ with the star's center as the pole and its axis of rotation as the polar axis). In a spherical system of coordinates, let us define $N$ and $L$ by $\partial / \partial r=i N, \partial / r \partial \theta=i L ; 1 / N$ and $1 / L$ are therefore the radial and latitudinal dimensions of the turbulence. At the equator, Equation (2.3.19) can then be written $\alpha\left[m^{2}+L^{2}\right]>4 \Omega_{0}^{2} L^{2}$; whereas at the poles, $\alpha\left[m^{2}+L^{2}\right]>4 \Omega_{0}^{2} N^{2}$. Therefore, turbulent convection will be preferentially stabilized at the poles if $N>L$ or $1 / N<1 / L$, that is, if the convective cells are flattened in the radial direction; if, on the other hand, the turbulent motions are elongated in the radial direction, then it is the convection at the equator that will be preferentially stabilized by rotation. The shape of the convective cells is presumably 
determined by complex phenomena such as nonlinear interactions of the velocity and temperature fields, the variations of density with height, and the action of rotation on the convective motions; it is not known at present whether the convective cells are elongated or flattened in the radial direction. (It should be noted, however, that Simon and Weiss, 1968, and Parker, 1973a, 1973b have argued that the convective motions, in the absence of rotation, extend over several scale heights.) Very little is also known about the effect of nonuniform rotation on the turbulence (cf. Cowling, 1951).

We return now to the results of the numerical calculations pertaining to the solutions of Equations (D3), (D4) and the linearized versions of Equations (E1) and (E2) (as stated above $s=1, \gamma=\frac{5}{3}, \nu=2 \times 10^{12} \mathrm{~cm}^{2} \mathrm{~s}^{-1}$ ). In Figures $6 \mathrm{a}$ and $6 \mathrm{~b}, \omega_{0}(r)$ and $\omega_{2}(r)$ are plotted against $r ; \omega_{0}(r)$ and $\omega_{2}(r)$ are in excellent agreement with previous calculations (Durney, 1974a); it should be noted that $\omega_{0}(r)$ increases with depth, whereas $\omega_{2}(r)$ decreases inward. This appears to be a typical behavior of the solutions of Equations (E1) and (E2) (with $s=1$ and free stress boundary conditions at $r=R_{c}, R_{0}$ ) when $\omega_{0}(r)$ and $\omega_{2}(r)$ are slowly varying functions of $r$ (cf. Section 3).

The pole-equator differences in flux are small: $\Delta \mathscr{F} / \mathscr{F}_{0} \sim 10^{-3}$ in the bulk of the convection zone and $\sim 10^{-6}$ in the surface layers. We have neglected here the energy carried by the meridional motions; if this energy is important we would expect two cells to develop in the radial direction (Durrey, 1972a): assume that convection is preferentially stabilized at the poles by rotation. In the lower part of the convection zone where the effect of rotation on convection is large, meridional motions should be generated that will increase the equatorial flux; these motions should therefore

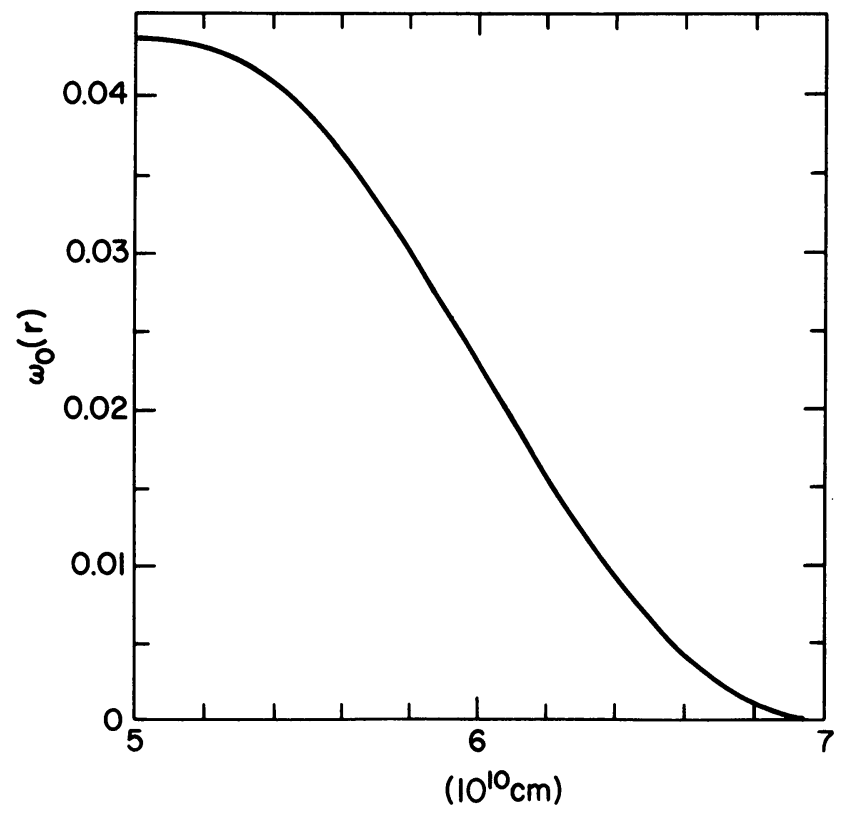

Fig. 6a. $\omega_{0}(r)$ versus depth. The angular velocity is given by $\Omega=\Omega_{0}\left(1+\omega_{0}(r)+\omega_{2}(r)\left(3 \cos ^{2} \theta-1\right) / 2\right)$. 


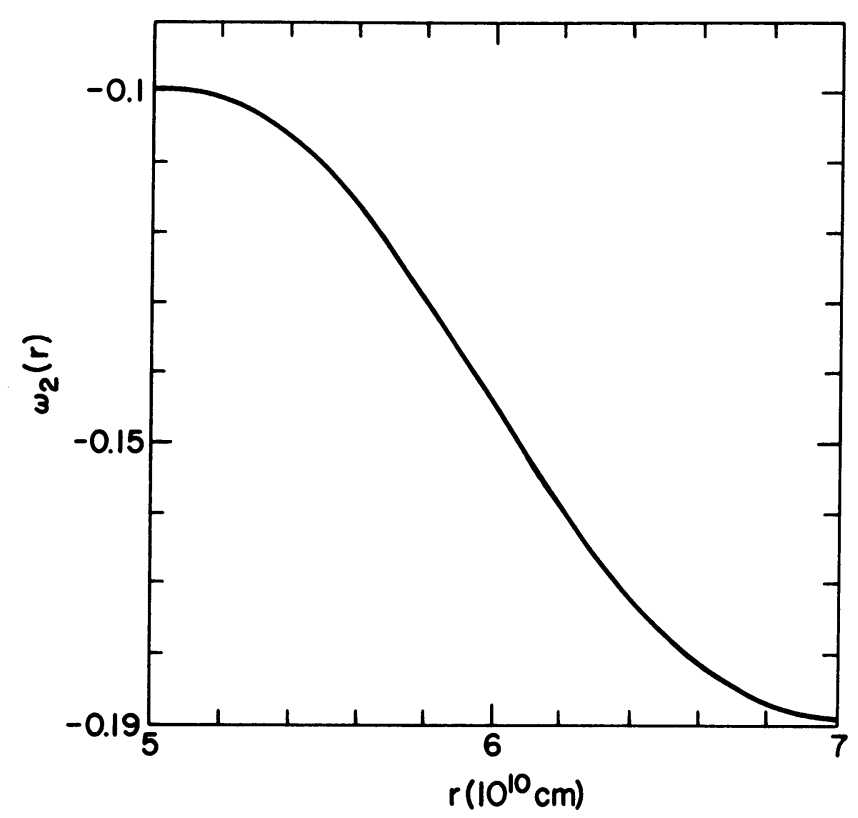

Fig. 6b. $\omega_{2}(r)$ versus depth.

rise at the equator and sink at the poles. In the upper part of the convection zone the effect of rotaiion on convection becomes negligible and a counter cell should develop (motions sinking at the equator and rising at the poles) as the convection zone relaxes to a state in which the effect of rotation is unimportant, i.e., to a nonrotating state. This counter cell redistributes the pole-equator differences in flux set up in the lower part of the convection zone, and the direction of these motions is such that they give rise to differential rotation with equatorial acceleration (Kippenhahn, 1963). It should be noted that if only one cell is present and the motions rise at the poles and sink at the equator, then the equatorial flux can be larger than the polar flux at the base of the convection zone $\left(r=R_{c}\right)$ if the meridional motions penetrate (even slightly) into the radiative region. We take $\left(\mathscr{F}_{r}\right.$ is the latitudinally dependent component of the radial flux)

$$
\mathscr{F}_{r}=\mathscr{F}(r) P_{2}(\cos \theta) ; \quad U_{r}=\frac{2 \psi(r)}{\rho r^{2}} P_{2}(\cos \theta)
$$

and approximate the energy equation by

$$
\mathrm{d} r^{2} \mathscr{F}_{r} / \mathrm{d} r=r^{2} c_{p} \rho U_{r}(\nabla \Delta T)_{u} .
$$

Since in the stable radiative core $(\nabla \Delta T)_{u}<0$, the left-hand side of the above equation will be larger than zero if $U_{r}<0$ (as we have assumed to be the case at the equator). Therefore in the radiative region downward motions transport energy upward and, in consequence, the equatorial flux will be larger than the polar flux at the base of the convection zone $\left(r=R_{c}\right)$ if the energy flux is spherically symmetric inside the radiative core. It should also be noted that since $|\nabla \Delta T|$ is 'large' in the radiative region, very small velocities can carry large amounts of energy flux. 
The meridional motions in the convection zone which are needed to generate the observed solar differential rotation are of the order of $U_{r} \sim 10 \mathrm{~cm} \mathrm{~s}^{-1}$ (Durney, 1974a). The same value is obtained from the solution of Equations (D3) and (D4). It is of interest to calculate the energy flux carried by these motions. We assume again that the radial components of the energy flux and velocity field are given by Equation (2.3.20) and that the energy equation is given by Equation (2.3.21). Since $\nu=l u_{c} / 3$, an integration of Equation (2.3.21) gives

$$
\delta \mathscr{F}_{r}=\mathscr{F}_{r}\left(r=R_{0}\right)-\frac{R_{c}^{2}}{R_{0}^{2}} \mathscr{F}_{r}\left(r=R_{c}\right)=\frac{2}{3} \mathscr{F}_{0} \int_{R_{c}}^{R_{0}}\left(U_{r} / \nu\right) \mathrm{d} r .
$$

If we take $v=2 \times 10^{12} \mathrm{~cm}^{2} \mathrm{~s}^{-1}$ and $U_{r}(r)=10 \mathrm{~cm} \mathrm{~s}^{-1}$, we obtain

$$
\delta \mathscr{F}_{r} / \mathscr{F}_{0} \sim \int_{R_{c}}^{R_{0}}\left(U_{r} / \nu\right) \mathrm{d} r \sim P_{2}(\cos \theta) / 10 .
$$

If $\mathscr{F}_{r}\left(r=R_{0}\right)=0$ (no pole-equator difference in flux at the surface) then Equation (2.3.22) shows that the pole-equator difference in flux at $r=R_{c}$ is the order of $\Delta \mathscr{F} / \mathscr{F} \sim \frac{1}{10}$. The values of $\Delta \mathscr{F} / \mathscr{F}$ found from the solution of Equations (D3), (D4), (E1), and (E2) are smaller than $\frac{1}{10}$ : the value used for the turbulent viscosity $\left(2 \times 10^{12} \mathrm{~cm}^{2} \mathrm{~s}^{-1}\right)$ is too small to justify neglecting the energy flux carried by the meridional motions. There can be little doubt that the terms of the energy equation that were neglected in Equation (2.3.21) would lower the values of $\Delta \mathscr{F} / \mathscr{F}$. Equation (2.3.22) suggests, however, that in this theory of differential rotation, the value of $\Delta \mathscr{F} / \mathscr{F}$ has to be significant at the base of the convection zone.

It is apparent from Figure $6 \mathrm{~b}$ that $\left|\omega_{2}(r)\right|$ decreases slowly inward. As a consequence, the large viscosity approximation is not valid in the lower part of the convection zone. We assume conservatively large values for $U_{r}^{\prime} \sim \rho^{\prime} U_{r} / \rho ; U_{\theta} \sim$ $r \rho^{\prime} U_{r} / \rho ; U_{\theta}^{\prime} \sim\left(r \rho^{\prime} / \rho\right)^{2} U_{r} / r$. It is readily seen from Equations (2.3.1) and (2.3.2) that the largest viscous terms of the $r$ and $\theta$ equations of motions are $V_{r}=\left(r \rho^{\prime} / \rho\right)^{2} \nu U_{n} \rho / r^{2}$ and $V_{\theta}=\left(r \rho^{\prime} / \rho\right)^{3} \nu U_{r} \rho / r^{2}$, respectively; the largest terms containing the meridional velocities are $M_{r}=\left(r \rho^{\prime} / \rho\right)^{2} U_{r}^{2} \rho / r$ and an identical expression for the $\theta$-equation $\left(M_{\theta}=M_{r}\right)$. In Table II the ratios $2 \Omega_{0}^{2} \rho\left|\omega_{2}\right| r / V_{r}$ and $2 \Omega_{0}^{2} r\left|\omega_{2} / V_{\theta}\right|$ are tabulated as a

TABLE II

Ratio of the Coriolis force to the viscous terms as a function of depth in the radial and latitudinal equations of motion

\begin{tabular}{llllll}
\hline$d(\mathrm{~cm})$ & $3.4(6)$ & $1.3(7)$ & $3.7(7)$ & $1.5(8)$ & $3.6(8)$ \\
$r$-Eq & $2.6(-1)$ & $2.9(-1)$ & 2.5 & $4.4(1)$ & $2.5(2)$ \\
$\theta-\mathrm{Eq}$ & $8.4(-6)$ & $3.6(-5)$ & $8.7(-4)$ & $6.3(-2)$ & $8.7(-1)$ \\
$\left|r \rho^{\prime} / \rho\right|$ & $3.1(4)$ & $8.1(3)$ & $2.8(3)$ & $7.0(2)$ & $2.9(2)$ \\
\hline & & & & & \\
\hline$d(\mathrm{~cm})$ & $1.0(9)$ & $3.3(9)$ & $1.1(10)$ & $1.8(10)$ & $1.9(10)$ \\
$r-\mathrm{Eq}$ & $1.3(3)$ & $7.2(3)$ & $4.2(4)$ & $1.3(5)$ & $2.1(5)$ \\
$\theta-\mathrm{Eq}$ & $1.2(1)$ & $2.4(2)$ & $5.3(3)$ & $2.9(4)$ & $5.1(4)$ \\
$\left|r \rho^{\prime} / \rho\right|$ & $1.0(2)$ & $3.0(1)$ & 8 & 4.3 & 4 \\
\hline
\end{tabular}


function of $r$ for $U_{r}=10 \mathrm{~cm} \mathrm{~s}^{-1}$ and $\left|\omega_{2}\right|=0.1$; the value of $\nu$ was taken from Table I and $r \rho^{\prime} / \rho$ was calculated by assuming that $\rho$ is given by a polytropic state (cf. Equations (2.3.14)) with $\gamma=\frac{5}{3}$. The values of $p_{u c}, \rho_{u c}, T_{u c}$ (the pressure, density, and temperature at the bottom of the convection zone) are from Baker and Temesvary's tables, and the value of gravity in Equation (2.3.15) was chosen so that $L=$ $1.9 \times 10^{10} \mathrm{~cm}\left(g=3.7 \times 10^{4} \mathrm{~cm}^{2} \mathrm{~s}^{-1}\right)$. The ratios of the Coriolis force to $M_{r}$ and $M_{\theta}$ behave very much in the same way as do the values listed in Table II for the $r$-equation since $U_{r}$ and $\nu / r$ are of the same order. It is clear from Table II that the large viscosity approximation is valid only in the surface layers of the convection zone. It is important, however, to realize that the results plotted in Figures (6a) and (6b) and the corresponding values of the meridional motions are more general than the large viscosity approximation: if the angular velocity is a slowly varying function of $r$ and the azimuthal stresses vanish at $r=R_{c}, R_{0}$, then $\omega_{0}(r)$ and $\omega_{2}(r)$ will behave very much as in Figure 6 for motions rising at the poles and sinking at the equator (see Section 3.1.).

\subsection{ROTATION IN CYLINDERS}

If differential rotation penetrates deeply into the convection zone, then the large viscosity approximation considered in the previous section is not valid. In fact, the inviscid radial and latitudinal equations of motion appear to be better approximations (Gierasch, 1974). It is not possible at present to rule out the possibility that differential rotation is a surface effect. The opposite appears more likely, however. (i) According to Parker (1975), the solar dynamo must be driven in the lower part of the convection zone; in the upper part magnetic buoyancy is so efficient that the magnetic field rapidly floats to the surface. (ii) according to Yoshimura (1975a), a latitudinal differential rotation must be present for the solar cycle models to reproduce the observed butterfly diagram.

We will assume, therefore, that differential rotation is not a surface phenomenon and that $\omega_{2} \sim=-0.1$ is a typical value of $\omega_{2}(r)$ in the lower part of the convection zone.

It was seen in the last section that for all reasonable values of an axisymmetric velocity field, the viscous terms and the terms in $U_{r}$ and $U_{\theta}$ in the $r$ - and $\theta$-equations of motion can be neglected in the lower part of the convection zone (Gierasch, 1974; the reader is referred to this paper for an order-of-magnitude estimate of all terms in these equations).

Global convection is essentially non-axisymmetric. We estimate now the largest values of the large-scale convective velocities $\left(U_{r}^{c}, U_{\theta}^{c}, U_{\phi}^{c}\right)$ such that the terms in $U_{r}^{c}$, $U_{\theta}^{c}, U_{\phi}^{c}$ of the $r$-and $\theta$-equations of motion are nevertheless smaller than the Coriolis force. As a guide to the relative magnitude of $U_{r}^{c}, U_{\theta}^{c}$, and $U_{\phi}^{c}$, we use Equations (B1) of Appendix II: $U_{r}^{c} \sim U_{\phi}^{c}$ and $U_{\theta}^{c}$ somewhat smaller than $U_{r}^{c}$ or $U_{\phi}^{c}$. For the derivatives with respect to $r, \theta$, and $\phi$ we take $\partial / \partial r \sim \rho^{\prime} / \rho ; \partial / r \partial \theta \sim L / r ; \partial / r \partial \phi \sim L / r$; an appropriate value of $L$ is $\sim 10$ (Appendix I). In consequence, $r \rho^{\prime} / \rho \geq L$ since in the lower part of the convection zone $r \rho^{\prime} / \rho \sim 5$ and $r \rho^{\prime} / \rho$ increases with $r$. Therefore, $\partial / \partial r \geqslant(\partial / r \partial \theta, \partial / r \partial \phi)$. The Navier-Stokes equations for the case of nonaxial symmetry have been written down, for example, by Pai (1956). It is readily seen that for 
the $r$-equation: the largest viscous term is $V_{r}=\left(r \rho^{\prime} / \rho\right)^{2} \nu U_{r}^{c} \rho / r^{2}$ and that the largest inertial terms containing the global convective velocities are $I_{1 r}=\rho^{\prime}\left(U_{r}^{q}\right)^{2}$ and $I_{2}=2 \rho \Omega_{0} U_{\phi}^{c}$. For the $\theta$-equation the largest viscous term is $V_{\theta}=\left(r \rho^{\prime} / \rho\right)^{2} \nu U_{\theta}^{c} \rho / r^{2}$ and the largest intertial terms (apart from the Coriolis term, $2 \Omega_{0}^{2} \rho \omega_{2} r$ ) are $I_{1 \theta}=$ $\rho^{\prime} U_{r}^{c} U_{\theta}^{c}$ and $I_{2}=2 \rho \Omega_{0} U_{\phi}^{c}$.

In Table II ( $r$-equation), the ratio of the Coriolis force $\left(2 \Omega_{0}^{2} \rho\left|\omega_{2}\right| r\right)$ to $V_{r}$ and $V_{\theta}$ are tabulated for $U_{r}^{c}=U_{\theta}^{c}=10 \mathrm{~cm} \mathrm{~s}^{-1}$. It is clear that even if $U_{r}^{c}$ and $U_{\theta}^{c}$ are as large as $10^{4} \mathrm{~cm} \mathrm{~s}^{-1}$ (larger, therefore, than the turbulent convective velocities; cf. Table I), the viscous terms due to the global convection can be neglected in the $r$ - and $\theta$-equations (in the lower part of the convection zone).

Consider now the inertial terms. The values of $U_{r}^{c}$ and $U_{\phi}^{c}$ (such that $I_{1 r} \sim I_{1 \theta}$ and $I_{2}$ are of the order of the Coriolis force, $\left.2 \rho \Omega_{0}^{2} \omega_{2} r\right)$ are $U_{r}^{c} \sim 2 \times 10^{4} \mathrm{~cm} \mathrm{~s}^{-1}$ and $U_{\phi}^{c} \sim 10^{4} \mathrm{~cm} \mathrm{~s}^{-1}$ (we have used $r \rho^{\prime} / \rho=10 ;\left|\omega_{2}\right|=0.1$, and $r=6 \times 10^{10} \mathrm{~cm}$ ). Therefore if the global convective velocities are not larger than the turbulent velocities, then the viscous and inertial terms due to the large-scale convection can be neglected, in the rand $\theta$-equations, in the lower part of the convection zone. Combining this result with the previous one pertaining an axisymmetric circulation, we conclude that in the lower part of the convection zone the $r$ - and $\theta$-equations of motion can be written

$$
\begin{aligned}
& \rho U_{\phi}^{2} / r-\rho g=\partial p / \partial r \\
& \rho U_{\phi}^{2} \operatorname{cotg} \theta / r=\partial p / r \partial \theta .
\end{aligned}
$$

Equations (2.4.1) and (2.4.2) were used by Gierasch (1974) in his work on differential rotation.

We evaluate now the perturbations in the convective flux due to differential rotation. The convective flux and the superadiabatic gradient are given by Equation (2.2.1). Since $u_{c}=l / 2(g \cdot \nabla \Delta T / T)^{1 / 2}$ we can write

$$
\mathscr{F}_{r}=\frac{l^{2}}{4} g^{1 / 2} \rho c_{p} A_{r}^{3 / 2} / T^{1 / 2} ; \quad \mathscr{F}_{\theta}=\frac{l^{2}}{4} g^{1 / 2} \rho c_{p} A_{r}^{1 / 2} A_{\theta} / T^{1 / 2}
$$

with

$$
A_{r}=(1-1 / \gamma) \frac{T}{p} \frac{\partial p}{\partial r}-\frac{\partial T}{\partial r} ; \quad A_{\theta}=(1-1 / \gamma) \frac{T}{p r} \frac{\partial p}{\partial \theta}-\frac{1}{r} \frac{\partial T}{\partial \theta}
$$

The absence of rotation defines the unperturbed state designated by a sub-index, $u$. We neglect second-order terms in the perturbation (rotation). The superadiabatic gradient is 'small'. Therefore, we expect the perturbations in the superadiabatic gradient to be the ones that are the most important, i.e. $\delta \nabla \Delta T /(\nabla \Delta T)_{u} \gg \delta p / p_{u}$, $\delta \rho / \rho_{u}, \delta T / T_{u}$. We take, therefore,

$$
\mathscr{F}_{r}=\frac{l_{u}^{2}}{4} g^{1 / 2} \rho_{u} c_{p} A_{r}^{3 / 2} / T_{u}^{1 / 2} ; \quad \mathscr{F}_{\theta}=\frac{l_{u}^{2}}{4} g^{1 / 2} \rho_{u} c_{p} A_{r u}^{1 / 2} A_{\theta} / T_{u}^{1 / 2}
$$

It is important to keep in mind that we neglected the effect of rotation on $l$. With the help of Equations (2.4.1) and (2.4.2) it is readily found that $A_{r}$ and $A_{\theta}$ can be written

$$
\begin{aligned}
& A_{r}=\left(-g+U_{\phi}^{2} / r\right) / c_{p}-\partial T / \partial r \\
& A_{\theta}=U_{\phi}^{2} \operatorname{cotg} \theta / r c_{p}-(1 / r) \partial T / \partial \theta .
\end{aligned}
$$


To evaluate $A_{r}$ and $A_{\theta}$ we need to calculate $\partial T / \partial r$ and $\partial T / \partial \theta$ as given by Equations (2.4.1) and (2.4.2), and the gas equation. Equations (2.4.1) and (2.4.2) will be solved with the help of expansions of the form

$$
\begin{aligned}
U_{\phi} & =\Omega_{0} r \sin \theta\left(1+\omega_{0}(r)+\omega_{2}(r) P_{2}(\cos \theta)\right) ; \\
p & =p_{u}+p_{0}+p_{2} P_{2}(\cos \theta)+p_{4} P_{4}(\cos \theta) ; \quad \rho=\rho_{u}+\cdots ; \\
T & =T_{u}+\cdots .
\end{aligned}
$$

Differential rotation will be assumed to be small, that is $\omega_{0}^{2}, \omega_{2}^{2}$, and $\omega_{0} \omega_{2}$ will be neglected (as well, of course, as the products of $\left.p_{i}, \rho_{i}, T_{i}(i=0,2,4)\right)$. With the help of these approximations we can evaluate $T$ in terms of the unperturbed state and of $\omega_{0}(r)$ and $\omega_{2}(r)$. The mathematical details are given in Appendix VI.

The perturbed spherically symmetric part of $A_{r}\left[-\frac{2}{3}\left(\Omega_{0}^{2} r / c_{p}\right)\left(1+2 \omega_{0}-2 \omega_{2} / 5\right)-\right.$ $\left.\partial T_{0} / \partial r\right]$ must be zero since rotation cannot alter the emerging flux. This is just an equation for $\partial T_{0} / \partial r$. To evaluate the remaining terms of $A_{r}$ and $A_{\theta}$, we approximate the unperturbed state by a polytrope (Equations (2.3.14) and (2.3.15)); in this case the following equality holds:

$$
\mu / R_{g}+\rho_{u}^{\prime} T_{u} / g \rho_{u}=1 / c_{p} .
$$

Substituting Equations (F1), (F2), and (F4) of Appendix VI into Equations (2.4.6) and (2.4.7), we obtain, with the help of Equation (2.4.9):

$$
\begin{aligned}
& A_{r}=\frac{2}{7} \frac{\Omega_{0}^{2} T_{u}}{g}\left[\frac{1}{3} \frac{d}{\mathrm{~d} r}\left(r B_{1}\right) P_{2}+\frac{3}{5} \frac{d}{\mathrm{~d} r}\left(r B_{2}\right) P_{4}\right] \\
& A_{\theta}=\frac{2}{7} \frac{\Omega_{0}^{2} T_{u}}{g}\left[\frac{1}{3} B_{1} \frac{\partial P_{2}}{\partial \theta}+\frac{3}{5} B_{2} \frac{\partial P_{4}}{\partial \theta}\right]
\end{aligned}
$$

where

$$
B_{1}=12 \omega_{2}+r \omega_{2}^{\prime}+7 r \omega_{0}^{\prime}, \quad B_{2}=r \omega_{2}^{\prime}-2 \omega_{2} .
$$

The radial and latitudinal components of the convective flux are given by Equation (2.4.5). In Table III we list the values of $\Delta \mathscr{F}=\left(l_{u}^{2} / 4\right) g^{1 / 2} \rho_{u} c_{p} A_{r u}^{1 / 2} \frac{3}{2}\left[\frac{2}{7}\left(\Omega_{0}^{2} T_{u} / g\right)\right] /$ $T_{u}^{1 / 2}=\frac{3}{2} \mathscr{F}\left[\frac{2}{7}\left(\Omega_{0}^{2} T_{u} / g\right) /(\nabla \Delta T)_{u}\right]$ as a function of depth; $\Delta \mathscr{F}$ gives an indication of the pole-equator differences in flux if the terms inside the bracket of Equation (2.4.10) do not vanish. Furthermore, $\mathscr{F}_{\theta} \sim \Delta \mathscr{F}$ if the terms inside the bracket of Equation (2.4.11) do not vanish. It is impossible to believe that such large values of $\Delta \mathscr{F}$ and $\mathscr{F}_{\theta}$

TABLE III

Values of $\Delta \mathscr{F}=\frac{3}{2} \mathscr{F}\left[2 \Omega_{0}^{2} T_{u} / 7 g(\nabla \Delta T)_{u}\right]$ as a function of depth $d$

\begin{tabular}{llllll}
\hline$d(\mathrm{~cm})$ & $3.4(6)$ & $1.3(7)$ & $3.7(7)$ & $1.5(8)$ & $3.6(8)$ \\
$\Delta \mathscr{F}\left(\mathrm{erg} \mathrm{cm}^{-2} \mathrm{~s}\right)$ & $2.4(1)$ & $5.3(2)$ & $1.2(3)$ & $1.4(4)$ & $1.8(5)$ \\
\hline \multicolumn{7}{l}{} & & & & \\
\hline$d(\mathrm{~cm})$ & $1.0(9)$ & $3.3(9)$ & $1.1(10)$ & $1.8(10)$ & $1.9(10)$ \\
$\Delta \mathscr{F}\left(\mathrm{erg} \mathrm{cm}^{-2} \mathrm{~s}\right)$ & $8.8(6)$ & $6.0(8)$ & $2.8(10)$ & $7.8(10)$ & $6.3(10)$ \\
\hline
\end{tabular}


could exist inside the convection zone. We conclude, therefore that $B_{1}$ and $B_{2}$, given by Equation (2.4.12), must be small. If this is the case then

$$
\omega_{2}(r)=\omega_{2}\left(R_{c}\right)\left(r / R_{c}\right)^{2} ; \quad \omega_{0}(r)+\omega_{2}(r)=\text { constant }
$$

and (we take the constant appearing in Equation (2.4.13) as zero).

$$
\Omega=\Omega_{0}\left(1-\frac{3}{2} \omega_{2}\left(R_{c}\right)\left(r / R_{c}\right)^{2} \sin ^{2} \theta\right) .
$$

Therefore the angular velocity is constant along cylinders. What has been shown is the following: If in the absence of rotation the convection zone is adiabatic, and if Equations (2.4.1) and (2.4.2) are good approximations for the radial and latitudinal equations of motion, then in the lower half of the convection zone the perturbations in the convective flux (produced by differential rotation) are unacceptably large unless the angular velocity is constant along cylinders. (Of course, this result holds only if the value of the latitudinal differential rotation is not too small in the lower half of the convection zone; we have assumed also that $l$ is not a function of $\theta$.)

Intuitively this result can be seen as follows: assuming that $A_{r}=A_{\theta}=0$, by cross differentiation of Equations (2.4.6) and (2.4.7) we obtain ( $g$ and $c_{p}$ are constants)

$$
\frac{1}{r} \frac{\partial}{\partial \theta} U_{\phi}^{2}-\frac{\partial}{\partial r} \operatorname{cotg} \theta U_{\phi}^{2}=0 \text {. }
$$

The general solution of this equation is $U_{\phi}=f(r \sin \theta)$. This, of course, is not surprising: if $A_{r}=A_{\theta}=0$ then the structure of Equations (2.4.6) and (2.4.7) is the same as that of the radial and latitudinal equations of motion of an incompressible rotating fluid. It is well known that in this case $\Omega$ must be constant along cylinder (Taylor-Proudam theorem). If $\Omega$ is not constant along cylinders then we expect $A_{r}$ and $A_{\theta}$ to be large because in the unperturbed, nonrotating state, the superadiabatic gradient is 'small': Therefore any small perturbation can nevertheless give rise to large perturbations in the superadiabatic gradient.

It should be stressed that it has not been shown that rotation in cylinders is a consequence of the energy and momentum equation. Whether this is indeed the case is an important question that remains to be settled. If the meridional motions are given by Equations (2.3.7), the problem is then to solve Equations (2.4.1) (2.4.2), the azimuthal equation (2.3.3), and the energy Equation (2.2.3). If $\Omega(r, \theta)$ is given by Equation (2.4.14), then the azimuthal equation of motion (2.3.3) determines the meridional velocities. An estimate of these velocities can be obtained from Equation (F6) of Appendix VI. If $\omega_{2}\left(R_{c}\right)=-0.1$ and $\xi(r \sin \theta)=0$, then $U_{r} \sim 40 \mathrm{~cm} \mathrm{~s}^{-1}$ at $r=6.2 \times 10^{10} \mathrm{~cm}$. Let $\delta \mathscr{F}$ be the perturbation in the energy flux $\left(\delta \mathscr{F}=\frac{3}{2} \mathscr{F A} /(\nabla \Delta T)_{u}\right.$ with $A_{r}$ and $A_{\theta}$ given by Equations (2.4.10) and (2.4.11)). It is readily seen that

$$
\begin{gathered}
\delta \mathscr{F}_{r}=\frac{45}{28} \frac{\nu \Omega_{0}^{2} p_{u}}{g} B_{r} \\
\delta \mathscr{F}_{\theta}=\frac{45}{28} \frac{\nu \Omega_{0}^{2} p_{u}}{g} B_{\theta}
\end{gathered}
$$

where $B_{r}$ and $B_{\theta}$ are the expressions in brackets in Equations (2.4.10) and (2.4.11). If $B_{r}$ and $B_{\theta}$ are not 'small' $\left(B_{r} \sim B_{\theta} \sim 1\right)$ then, since the pressure is a strongly varying 
function of $r$,

$$
\operatorname{div} \delta \mathscr{F}=-\frac{45}{28} \nu \Omega_{0}^{2} \rho_{u} B_{r}
$$

and the energy equation (Equation 2.2.3) becomes

$$
-\frac{45}{28} \nu \Omega_{0}^{2} \rho_{u} B_{r} \sim c_{p} \rho_{u} U_{r}(\nabla \Delta T)_{u}=\mathscr{F} U_{r} / \frac{3}{2} \nu .
$$

At $r=6 \times 10^{10} \mathrm{~cm}, \nu \sim 10^{13} \mathrm{~cm}^{2} \mathrm{~s}^{-1}$ and $\rho_{u} \sim 7.6 \times 10^{-2} \mathrm{~g} \mathrm{~cm}^{-3}$. For the left- and right-hand sides of Equation (2.4.19) we obtain $10 B_{r}$ and 0.23 cgs units, respectively (we used $U_{r}=40 \mathrm{~cm} \mathrm{~s}^{-1}$ ). Therefore, even for values of $U_{r}$ as large as $40 \mathrm{~cm} \mathrm{~s}^{-1}$, the energy equation appears to favor small values of $B_{r}$. If $B_{r}$ is small, then $r B_{1}$ and $r B_{2}$ must be slowly varying functions of $r$; we take

$$
r B_{1}=\beta R_{c} ; \quad r B_{2}=\alpha R_{c}
$$

with $\alpha$ and $\beta$, two constants. It is readily seen that the solutions of Equations (2.4.20) are given by

$$
\omega_{2}(r)=-\frac{\alpha}{3}\left(\frac{R_{c}}{r}\right)+\xi\left(r / R_{c}\right)^{2} ; \quad \omega_{0}(r)=\frac{-(11 \alpha+3 \beta)}{21} \frac{R_{c}}{r}-\xi\left(\frac{r}{R_{c}}\right)^{2}
$$

where $\xi$ is constant.

If $\omega_{0}(r)$ and $\omega_{2}(r)$ are given by Equations (2.4.21) then the energy equation reduces to

$$
\delta \mathscr{F}_{\theta} / r \sim \mathscr{F} U_{r} / \frac{3}{2} \nu .
$$

At $r=6 \times 10^{10} \mathrm{~cm}$, the left- and right-hand side of Equation (2.4.22) are equal to 0.6 $B_{\theta}$ and $0.23 \mathrm{cgs}$ units, respectively $\left(U_{r}=40 \mathrm{~cm} \mathrm{~s}^{-1}\right)$. These quantities are of the same order if $B_{\theta} \sim 1$. In the present very crude analysis we have assumed that $\mathbf{U} \cdot \nabla \Delta T \sim$ $U_{r}(\nabla \Delta T)_{u}$ with $U_{r} \sim 40 \mathrm{~cm} \mathrm{~s}^{-1}$. It is clear that a much more careful study of this problem is needed before we can resolve whether the momentum and energy equations indeed favor a constant angular velocity along cylinders.

For the mixing length $l$ we have taken its unperturbed value; we have neglected therefore the latitudinal and radial dependence of the stabilizing effect of rotation on convection (cf. Equation 2.3.19). An estimate of the magnitude of this effect is an important problem that remains to be solved and it is not known at present if therein lies the solution to the heat flux problem. It is appealing, of course, to think that differential rotation arises as a way for the convection zone to minimize the constraints imposed by rotation on convection and so to maintain a uniform heat flux.

It is possible, perhaps to give a non-rigorous argument against the existence of large $\Delta \mathscr{F}$ 's inside the convection zone: in the surface layers $\left(d<\sim 3 \times 10^{9} \mathrm{~km}\right)$ the effect of rotation on turbulent convection becomes negligible and the convective flux should be well described by Equation (2.2.1), with $l$ the unperturbed mixing length. The values of $\Delta \mathscr{F}$ listed in Table III are small for $d<\sim 10^{9} \mathrm{~km}$. The existence of larger values of $\Delta \mathscr{F}$ would imply that Equations (2.4.1) and (2.4.2) are not good approximations at these depths; therefore, large velocities would have to be present in the Sun's surface layers.

It follows from Table III that in the upper part of the convection zone the perturbations in the convective flux are very small even if $\Omega$ is not constant along 
cylinders. In consequence, if $\Delta \mathscr{F}$ is negligible at a certain depth $\left(d \sim 2 \times 10^{4} \mathrm{~km}\right.$, for example), the energy equation will be approximately satisfied if $\psi=0$. It is of interest, therefore, to consider the solution of the equations for $\omega_{0}(r)$ and $\omega_{2}(r)$ (Equations (E1) and (E2) of Appendix V) in the case when $\psi=0$. In Table IV we list the values of $\omega_{0}(r)$ and $\omega_{2}(r)$ as a function of $r$ in the upper part of the convection zone for $\psi=0$, zero stresses at $r=R_{0}$ and $\omega_{2}\left(R_{0}\right)=-0.189$. (For the density a polytropic relation with $\gamma=5 / 3$ was used.)

Assume that differential rotation is generated in the surface layers of the Sun (by transport of angular momentum by the Reynolds stresses, for example) and that $\Delta \mathscr{F}$ and $\Delta T$ are very small at $r=R_{0}$; it would be of interest to know the dependence of $\Omega$ with depth if the momentum and energy equations are solved with boundary conditions expressing that $\Delta \mathscr{F}$ and $\Delta T$ are small at the surface and that $\omega_{2}(r)=$ -0.189 at $r=R_{0}$. If differential rotation is indeed a surface phenomenon, we expect that $\Omega$ will behave as follows: from Table IV $(s=1),\left|\omega_{2}(r)\right|$ should first increase inward; with increasing depth, meridional motions will be driven by the pole-equator differences in flux that will appear (cf. Table III). These meridional motions should be such as to decrease $\omega_{2}(r)$ and make it eventually vanish.

In any theory of the Sun's differential rotation, the value of $\Omega$ at the surface should, of course, agree with the observed differential rotation of the Sun. Furthermore, it is well known that theories of the solar cycle (Parker, 1955; Steenbeck and Krause, 1969; Leighton, 1969; Parker, 1971; Deinzer and Stix, 1971; Roberts and Stix, 1972; Lerche and Parker, 1972; Yoshimura, 1972, 1975a, b; Köhler, 1973) are sensitive to the values of $\mathrm{d} \Omega / \mathrm{d} \theta$ and to $\mathrm{d} \Omega / \mathrm{d} r$ in particular. The dependence of $\Omega$ on depth and latitude must therefore satisfy another important constraint: this dependence must be such that theories of the solar cycle reproduce the observed properties of the solar activity cycle. It could be thought that an angular velocity which decreased inward (as in the case if $\Omega$ is constant along cylinders) is necessarily in contradiction to theories of the solar cycle. However this is not so. To ensure that the solar dynamo displays the correct butterfly diagram (i.e., the mean toroidal field migrates towards the equator) the product $\alpha \partial \Omega / \partial r$ must be negative (Stix, 1974). According to Yoshimura (1975a), the $\alpha$-term (due to the global convection) changes sign in the lower part of the convection zone and is negative. Therefore rotation in cylinders could be compatible with Yoshimura's model of the solar cycle (Yoshimura, $1975 b$ ) if the observed toroidal magnetic field is generated in the lower part of the convection zone, as has recently been argued by Parker (1975). As discussed more fully by Stix (1976a, 1976b), however, the observations show that there is a phase relation between the toroidal and poloidal magnetic fields which could not be satisfied if $\Omega$ decreases inward.

\section{Anisotropic Viscosity and Normal Modes of Vibration}

\subsection{ANISOTROPIC VISCOSITY}

Since gravity in the solar convection zone is a preferred direction, there is no reason to expect that the turbulent viscosity in the directions parallel and perpendicular to 


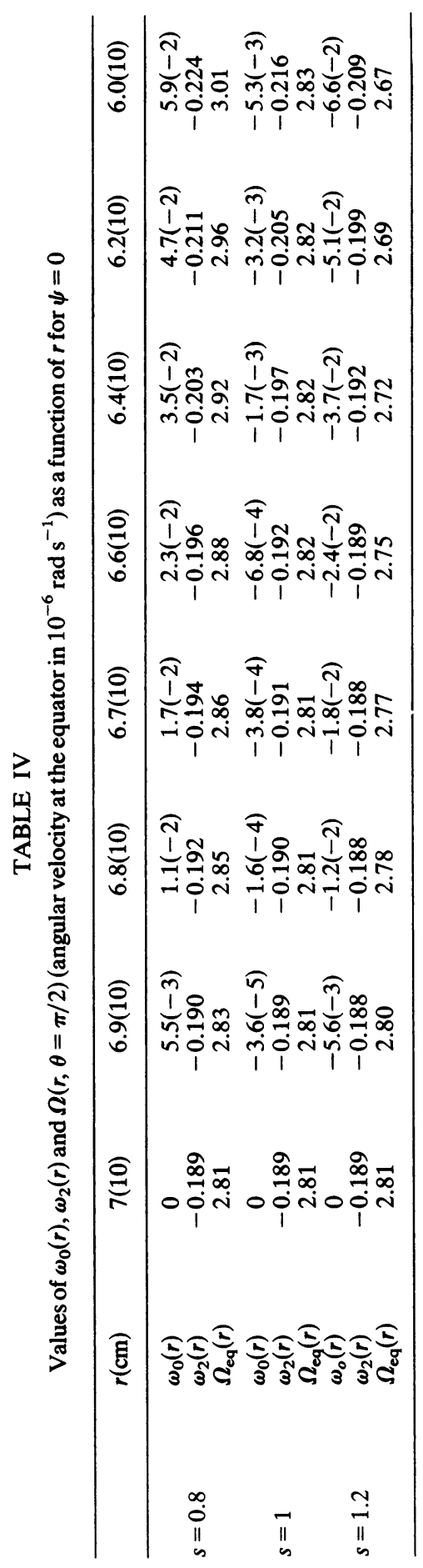


gravity will be the same. If the viscosity is anisotropic, the convection zone cannot rotate uniformly (Bierman, 1951); this theory of differential rotation has been developed by Kippenhahn (1963), Cocke (1967), Köhler (1970), Sakurai (1966), Iroshnikov (1969), Rüdiger (1974), (1976), Roxburgh (1974), and Durney (1974a). There can be little doubt about the soundness of the basic idea of this theory of differential rotation: the turbulent viscosity should indeed be anisotropic. The question that remains to be answered is how important this anisotropy is in generating the Sun's differential rotation.

The ratio of the kinematic turbulent viscosity coefficients in the directions perpendicular and parallel to gravity $\left(\nu_{\perp} / \nu_{\|}\right)$will be assumed to be a constant, independent of depth and latitude: therefore (Kippenhahn, 1963) $\nu_{\theta \theta}=\nu_{\phi \phi}=\nu_{\perp}=s \nu_{\|}=s \nu_{r r}=s \nu$.

We take the meridional motions and the angular velocity to be given by Equations (2.3.9) and (2.3.17), respectively; the equations for $\omega_{0}(r)$ and $\omega_{2}(r)$ are then Equations (E1), (E2), and (E3) of Appendix V. In the large viscosity limit, Equation (E2) is satisfied if $\omega_{2}=0$, and Equation (E1) gives then $\left(\rho r^{4} \omega_{0}^{\prime}\right)^{\prime}+$ $2(1-s)\left(r^{3} \rho\left(\omega_{0}+1\right)\right)^{\prime}=0$, or $\rho r^{4}\left(\omega_{0}^{\prime}+2(1-s)\left(1+\omega_{0}\right) / r\right)=$ constant. If the azimuthal stress, $T_{r \phi}$, vanishes at $r=R_{0}$, for example, then it is readily seen from Equation (C2c) of Appendix III that $\omega_{0}^{\prime}+2(1-s)\left(1+\omega_{0}\right) / r$ must also vanish. Therefore,

$$
\omega_{0}=\omega_{0}\left(R_{0}\right)\left(R_{0} / r\right)^{2(1-s)} .
$$

This rotation law replaces solid rotation when $s \neq 1$.

To understand some general properties concerning the behavior of $\omega_{0}(r)$ and $\omega_{2}(r)$, we write Equations (E1) and (E3) of Appendix V in the following form:

$$
\begin{aligned}
& \omega_{0}^{\prime}+2(1-s)\left(1+\omega_{0}\right) / r=\frac{2}{r^{4} \rho} \int_{R_{0}}^{r}\left[\omega_{2} s \rho r^{2}+\frac{\psi}{3 \nu} \frac{d}{d r}\left(r^{2}\left(1+\omega_{0}+2 \omega_{2} / 5\right)\right)\right] \mathrm{d} r \\
& -\frac{2 \psi}{3 \nu r^{2} \rho}\left(1+\omega_{0}-\omega_{2} / 5\right), \\
& 2 \psi / \nu=\frac{r^{2} \rho\left[\omega_{2}^{\prime}+2(1-s) \omega_{2} / r-5\left(\omega_{0}^{\prime}+2(1-s)\left(1+\omega_{0}\right) / r\right)\right]}{1+\omega_{0}-5 \omega_{2} / 7}
\end{aligned}
$$

where we have assumed that the azimuthal stress, $T_{\phi r}$, and the radial velocities vanish at $r=R_{0}$. If $\omega_{0}+2 \omega_{2} / 5$ is a slowly varying function of $r$, that is, if

$$
\left|\frac{d}{\mathrm{~d} r} r^{2}\left(\omega_{0}+2 \omega_{2} / 5\right)\right|<\frac{2}{r}
$$

then the term in $\psi$ in the integral of Equation (3.1.2) reduces to $2 r \psi / 3 \nu$. Neglecting $\omega_{0}$ and $\omega_{2} / 5$ with respect to one, Equation (3.1.2) becomes

$$
\omega_{0}^{\prime}+2(1-s)\left(1+\omega_{0}\right) / r=\frac{2}{r^{4} \rho} \int_{R_{0}}^{r} \rho r^{2}\left[\omega_{2} s+2 r \psi / 3 \nu \rho r^{2}\right] \mathrm{d} r-2 \psi / 3 \nu r^{2} \rho
$$

If the azimuthal stresses and radial velocities vanish at $r=R_{c}$, then the integral in 
Equation (3.1.5) must be zero for $r=R_{c}$. Equation (3.1.3) shows that if $\omega_{0}$ and $\omega_{2}$ are slowly varying functions of $r$, the same is true of $2 \psi / \nu \rho r^{2}$. The bracketed quantity in the integral of Equation (3.1.5) is then also a slowly varying function of $r$, and must therefore approximately vanish if the integral vanishes. This gives an estimate of $\omega_{2}$.

$$
\omega_{2} \sim-\frac{2 \psi}{3 \nu s \rho r} .
$$

For $\omega_{2}=-0.2$ and $s=1$, we obtain $\psi / r^{2} \rho \sim 10 \mathrm{~cm} \mathrm{~s}^{-1}$, in excellent agreement with previous calculations (cf. Figure 2a of Durney, 1974a). Equation (3.1.6) clearly shows that if $\omega_{2}<0$, then $\psi$ has to be larger than zero: the meridional motions must rise at the poles and sink at the equator.

To proceed further we need an estimate of the integral $(1 / \rho) \int_{R_{0}}^{r} \rho \mathrm{d} r$ which appears in Equation (3.1.5). For a polytrope, and with the exception of a thin layer at the top, we have $(1 / \rho) \int_{R_{0}}^{r} \rho \mathrm{d} r=(\gamma-1) / \gamma\left(r-R_{0}\right)$. Therefore, the term $\left(2 / r^{4} \rho\right) \int_{R_{0}}^{r} \rho r^{2} \omega_{2} s \mathrm{~d} r$ in Equation (3.1.5) is of the order of $4 s\left|\omega_{2}\right|\left(R_{0}-r\right) / 5 r^{2}<$ $4 s\left|\omega_{2}\right|\left(R_{0}-R_{c}\right) / 5 r^{2} \sim 4 s\left|\omega_{2}\right| / 25 r$ (where we have taken $\gamma=5 / 3$ ). Since the integral in Equation (3.1.5) must vanish for $r=R_{c}$, we expect its value to be smaller than $4 s\left|\omega_{2}\right| 25 r$. The last term of Equation (3.1.5) on the other hand, is of the order of $\left|\omega_{2}\right| s / r$. This suggests neglecting the integral in Equation (3.1.5) which then becomes

$$
\omega_{0}^{\prime}+2(1-s)\left(1+\omega_{0}\right) / r=-2 \psi / 3 \nu r^{2} \rho
$$

The integrated version of Equation (E3) in Appendix $V$ then gives

$$
\omega_{2}^{\prime}+2(1-s) \omega_{2} / r=-4 \psi / 3 \nu r^{2} \rho
$$

for $s=1$ and for a typical value of $\psi$ given by Equation (3.1.6), $\omega_{0}(r)$ and $\omega_{2}(r)$ behave very much as in Figures $6 \mathrm{a}$ and $6 \mathrm{~b}$; in particular $\omega_{2}(r)$ must increase inward since $\psi>0$, that is, the latitudinal variations of the angular velocity decrease with depth.

If differential rotation is generated by an anisotropy in the turbulence, it is well known that $s>1(s<1)$ results in a fast (slow) rotating equator (Kippenhahn, 1963). If $s>1$ the basic solution, in the limit of large viscosity, decreases inward (cf. Equation (3.1.1)). If $s=1$, Figure 6a or Equation (3.1.7) shows that $\omega_{0}^{\prime}<1$ (since $\psi$ has to be larger than zero); therefore $\omega_{0}$ increases inward. The behavior of $\omega_{0}(r)$ is important for theories of the solar dynamo.

It was stated in Section (1.1) that Stenflo's interpretation of the angular velocities plotted in Figure 1 was not without difficulties. Following Stenflo, we accept that the three curves labeled 'sign of longitudinal magnetic field, 'longitudinal magnetic field' and 'sunspots' in Figure 1 give an indication of the Sun's angular velocity at different depth in the convection zone.

In relation to differential rotation, we have come upon the fact in previous sections (2.3 and 2.4) that in the solar convection zone there appears to be two regions where different approximations hold: the surface layers and the lower part of the convection zone. This provides a natural interpretation of the two sets of curves in Figure 1: the curve labeled 'Doppler shifts' (set 1) and the other three curves (set 2) referring to the magnetized plasma could give an indication of the Sun's angular velocity in these 
two regions. If this is the case, a striking feature of the Sun's angular velocity in the deep layers of the convection zone is the constant angular velocity at the equator. It is of interest to note that this behavior is predicted by the approximate Equations (3.1.7) and (3.1.8) if $s=1$ : the angular velocity at the equator is given by $\Omega=$ $\Omega_{0}\left(1+\omega_{0}(r)-\omega_{2}(r) / 2\right)$; therefore, $\mathrm{d} \Omega / \mathrm{d} r=\Omega_{0}\left(\omega_{0}^{\prime}(r)-\omega_{2}^{\prime}(r) / 2\right)=0$ in virtue of Equations (3.1.7) and (3.1.8) $(s=1)$. Furthermore Table IV shows that if $s=1$ and $\psi=0$ the angular velocity at the equator is also very approximately constant. (In Table IV, the full equations for $\omega_{0}(r)$ and $\omega_{2}(r)$ were solved, i.e. Equations (E1) and (E2) of Appendix V.)

According to Parker (1975), the toroidal magnetic field associated with the solar activity cycle is generated in the lower part of the convection zone. It appears natural, therefore, to assume that in the three curves of 'set 2' in Figure 1, the larger is the magnetic field, the deeper in the convection zone is this field rooted. This would imply, however, that differential rotation $\left(\left|\omega_{2}(r)\right|\right)$ increases with depth and such behavior of $\omega_{2}(r)$ is very difficult to understand. It is certainly in disagreement with $\Omega$ being constant along cylinders. Figure 1 shows also that the angular velocity of the magnetized plasma is larger than the photospheric angular velocity. If $s=0.8$ and $\psi=0$, the angular velocity at the equator and $\left|\omega_{2}(r)\right|$ both increase inward (cf. Table IV). This solution is, however, unlikely to be valid outside the surface layers. Therefore, there could indeed be a region beneath the solar surface with larger equatorial angular velocity and differential rotation than at $r=R_{0}$. That this region could extend down to the lower part of the convection zone appears very difficult to understand.

Köhler (1970) has solved the momentum equations with anisotropic viscosity and constant density. For $\nu=4 \times 10^{12} \mathrm{~cm}^{2} \mathrm{~s}^{-1}$ the angular velocity is constant along cylinders as a consequence of the Taylor-Proudman theorem. The energy equation (and it is clear from previous sections that this equation is the source of all difficulties) has, however, not been included in theories of differential rotation based on an anisotropic turbulent viscosity.

\subsection{NORMAL MODES OF VIBRATION}

As stated in Section 1.6, evidence has been accumulating for the existence of normal mode of vibrations of the entire Sun. It is at present too early to judge the impact that their existence will have on theories of the large-scale circulation of the solar convection zone. This impact could be of great importance.

Radiative and viscous damping of these modes occurs rather close to the surface; they provide, therefore, a mechanism for a rapid transmission of energy directly from the Sun's core to the surface. Recently Hill et al. (1975) have suggested that a non-negligible fraction of the solar energy flux could be carried by these normal modes.

Theoretical studies of the Sun's normal mode of vibrations in view of explaining the rigid rotation of magnetic features and the Sun's differential rotation have been carried out by Wolff $(1974 a, b)$. According to Wolff the Sun's differential rotation could be generated as follows: under the influence of nonlinear coupling mechanisms, modes with the most similar rotation rates should lock together and rotate as a 
single entity (designated by $L$ hereafter). The power contained in the set of normal modes $(L)$ is not distributed uniformly over the solar surface but concentrated mainly into regions running diagonally across the surface from pole to pole in a $V$-shaped pattern (cf. Figure 1 of Wolff, 1974b). The dissipation of this power in the surface layers of the sun drives large-scale flows which could then generate the observed solar differential rotation.

Even if the explanation of the Sun's differential rotation is not the one suggested by Wolff, observational and theoretical work on the Sun's normal mode of vibrations could be of importance for the understanding of the solar differential rotation. It is obvious that a mechanism coupling the surface to inner regions of the Sun (which could rotate with different angular velocities) must not be overlooked.

\section{The Inner Rotation of the Sun}

This paper is concerned mainly with theories of the Sun's differential rotation. The very important problem of the solar inner rotation will be discussed only briefly; in fact, it may not be possible to study both problems independently.

The observational evidence which has been summarized in Sections (1.7)-(1.9), indicates that all stars arrive at the main sequence $(t=0)$ with large angular velocities (an angular velocity given by the line $\langle\mathfrak{M}\rangle^{2 / 3}$ of Figure 3 would perhaps be the most reasonable assumption). Whereas stars more massive than $\sim \mathrm{F} 6$ do not appear to lose much angular momentum during their stay in the main sequence, the evolution of stars less massive than $\sim \mathrm{F} 6$ is quite different: at $t=0$ they are rapid rotators and they must have rather large magnetic fields since they show strong $\mathrm{Ca}$ II emission (in the Sun Ca II emission is an indication of strong magnetic fields). Both the angular velocity and the magnetic field (Ca II emission) decrease with time. It is well known that stars less massive than $\sim$ F6 have appreciable surface convection zones; it appears reasonable, therefore, to assume that the magnetic field is generated by a dynamo action (as is the case for the Sun). All these observations can then be understood by invoking the angular momentum loss (due to the existence of stellar winds) experienced by stars with convection zones (Schatzman, 1962). This angular momentum loss slows down the star; as a consequence the dynamo action becomes less efficient and the magnetic field decreases. Skumanich (1972) has found that the time dependence of both $\Omega$ and $B$ are well described by $B \sim t^{-1 / 2}, \Omega \sim t^{-1 / 2}$ (for values of $t$ that are not too small; $t=0$ corresponds to the arrival of the star at the main sequence). Estimates of the angular momentum loss (Dicke, 1964; Modisette, 1967; Weber and Davis, 1967; Alfonso-Faus, 1967) show that

$$
\mathrm{d} J / \mathrm{d} t=\frac{2}{3} \Omega_{s} r_{\mathrm{A}}^{2} \mathrm{~d} M / \mathrm{d} t
$$

where $\mathrm{d} J / \mathrm{d} t$ and $\mathrm{d} M / \mathrm{d} t$ are the angular momentum and mass loss, $r_{\mathrm{A}}$ is Alfvenic distance, and $\Omega_{s}$ is the surface angular velocity. From the definition of $r_{\mathrm{A}}\left(B_{\mathrm{A}}^{2} / 4 \pi \rho_{\mathrm{A}} U_{\mathrm{A}}^{2}=1\right)$ we obtain $B_{\mathrm{A}}^{2} r_{\mathrm{A}}^{2} / U_{\mathrm{A}} 4 \pi \rho_{\mathrm{A}} U_{\mathrm{A}} r_{\mathrm{A}}^{2}=1$; since the mass loss is given by $\mathrm{d} M / \mathrm{d} t=-4 \pi \rho_{\mathrm{A}} U_{\mathrm{A}} r_{\mathrm{A}}^{2}$, it is readily seen that Equation (4.1) can be written

$$
\mathrm{d} J / \mathrm{d} t=-\frac{2}{3} \Omega_{s}\left(B_{0} R_{0}^{2}\right)^{2} / U_{\mathrm{A}}
$$


where $B_{0}$ is the surface magnetic field and $R_{0}$ is the radius of the $\operatorname{star}\left(B_{0} R_{0}^{2}=B_{\mathrm{A}} r_{\mathrm{A}}^{2}\right)$. Equation (4.2) shows that in the first approximation the angular momentum loss is proportional to $\Omega_{s} B_{0}^{2}$ : even for stars with such small magnetic fields as the Sun, $r_{\mathrm{A}}$ is much farther than the critical point and we can replace $U_{\mathrm{A}}$ by $U_{\infty}$. In other words, in Equation (4.2), $U_{\mathrm{A}}$ is not a sensitive function of the magnetic field. (The stellar wind velocities could change with the age of the star, but again we expect $\Omega_{s}$ and $B_{0}^{2}$ in Equation (4.2) to be much stronger functions of $t$ than $U_{\mathrm{A}}$.) The magnetic field is generated by a dynamo action and is therefore itself a function of $\Omega_{s}$ (cf. Spiegel, 1968). There is both observational (Skumanich, 1972) and theoretical (Roberts, 1974) evidence to suggest that $B_{0} \propto \Omega_{s}$. Equation (4.2) can then be written

$$
\mathrm{d} J / \mathrm{d} t=-\alpha \Omega_{s}^{3}
$$

where $\alpha$ can be determined from observations. A convenient way of expressing the present-day torque is in terms of $\tau_{0}=-\Omega_{0} /\left(\mathrm{d} \Omega_{0} / \mathrm{d} t\right)$, the $e$-folding time for slowing down the Sun rotating rigidly (Dicke, $1972 ; \Omega_{0}$ is the present surface angular velocity of the Sun). Observations show that present-day torque is such that $\tau_{0} \sim 10^{10} \mathrm{yr}$. If we assume that the sun rotates rigidly, Equation (4.3) becomes

$$
\frac{d}{\mathrm{~d} t}\left(\frac{\Omega_{s}}{\Omega_{0}}\right)+\frac{1}{\tau_{0}}\left(\frac{\Omega_{s}}{\Omega_{0}}\right)^{3}=0: \quad \tau_{0}=10^{10} \mathrm{yr} .
$$

(If the Sun rotates rigidly, $J$ is proportional to $\Omega_{s}$, Equation (4.3) must therefore be of the type $\mathrm{d} \Omega_{s} / \mathrm{d} t=-\beta \Omega_{s}^{3}$ with $\beta$ a constant; for the present-day Sun, Equation (4.4) gives $\tau_{0}=-\Omega_{0} /\left(\mathrm{d} \Omega_{0} / \mathrm{d} t\right)$ and this determines $\left.\beta\right)$. The solution of Equation (4.4) is

$$
\Omega_{s}=\Omega_{s}(t=0) /\left(1+2 t\left(\Omega_{s}(t=0) / \Omega_{0}\right)^{2} / \tau_{0}\right)^{1 / 2} .
$$

It is reasonable to assume that the Sun arrived at the main sequence rotating with an angular velocity given by the $\langle\mathfrak{M}\rangle)^{2 / 3}$ line of Figure 3 , that is (Durney, 1972), $\Omega_{s}(t=0)=65 \Omega_{0}$. In a time larger than $\tau_{0} /\left(2 \times 65^{2}\right) \sim 10^{6} \mathrm{yr}, \Omega_{s}$ as given by Equation (4.5) reduces to

$$
\Omega_{s}=\frac{\Omega_{0} \tau_{0}^{1 / 2}}{\sqrt{2}} t^{-1 / 2}
$$

which agrees well with the observational law found by Skumanich (1972). Furthermore, for the present-day Sun, $t=5 \times 10^{9} \mathrm{yr}=\tau_{0} / 2$, Equation (4.6) gives $\Omega_{s}=\Omega_{0}$. For $t=3 \times 10^{7} \mathrm{yr}$ and $t=4 \times 10^{8} \mathrm{yr}$ (the age of the Pleiades and Hyades) Equation (4.6) gives $\Omega_{s}=13 \Omega_{0}$ and $\Omega_{s}=3.5 \Omega_{0}$, respectively. The predicted angular velocity for the Hyades is in good agreement with observations (Kraft, 1967), but the predicted angular velocity for the Pleiades is somewhat high. In all, the predictions of such a simple equation as Equation (4.4) are surprising. The angular velocity at $t=0$ is not too important, since the angular momentum loss is initially very fast. In fact Equation (4.6) does not contain any information about the angular velocity at $t=0$; according to this equation, the $e$-folding time for the slowing down of the Sun is given by

$$
\tau_{s}=\Omega_{s} /\left(\mathrm{d} \Omega_{s} / \mathrm{d} t\right)=2 t
$$


Could the Sun have slowed down uniformly (due to the existence of a magnetic field, for example)? The observations of the SCLERA group (Hill et al., 1974; Hill and Stebbins, 1975a, b) indicate that the Sun has no appreciable quadrupole moment; they lend some support, therefore, for a uniform solar angular velocity. (The quadrupole moment is nevertheless a sensitive function of $\Omega$ (cf. Roxburgh, 1964) and the Sun's core could be rotating quite fast ( $\sim 1$ week) with the Sun still showing a small quadrupole moment.) However, there is one observation, namely the Li depletion at the surface of solar type stars (cf. Section 1.9), that could be in contradiction with a uniform angular velocity; Li depletion allows for an appealing explanation if the Sun's interior is rotating fast: the angular velocity increases inward and becomes unstable (Goldreich and Schubert, 1967; Fricke, 1968). This instability gives rise to a mild turbulence that mixes the Sun's matter down to $\sim 0.63 R_{0}$, where $\mathrm{Li}$ is destroyed (cf. Dicke, 1971; 1972).

The simplest explanation of $\mathrm{Li}$ depletion would certainly be in terms of the overshooting of the convective motions into the radiative region. If this were the case the observations concerning the $\mathrm{Li}$ depletion would be in agreement with a uniform angular velocity. Overshooting, which is larger than earlier estimates, appears to be a complex phenomenon requiring more sophisticated theories of the turbulent energy transport than those generally used in calculating stellar convection zones (cf. Shaviv and Salpeter, 1973). It must be stressed that at present no theory exists which explains the $\mathrm{Li}$ depletion in terms of convective overshooting.

However, even if the Sun is rotating uniformly, turbulence could be generated in the upper part of the radiative region by the latitudinal variations of the angular velocity. In the convection zone the variations with $r$ and $\theta$ of $\Omega$ are determined by processes that have no parallel in the radiative region. Assume, for example, that $\Omega$ is constant along cylinders in the convection zone. If Equations (2.4.1) and (2.4.2) are satisfied, the $\partial p / \partial \theta$ term is balanced mainly by Coriolis forces. As stressed by Gierasch (1974), rotation cannot be uniform for $r<R_{c}$ since then, as a consequence of $\partial p / \partial \theta$, an unbalanced radial gradient in the pressure would appear. Differential rotation must therefore penetrate into the radiative region. Because of the low viscosity of this region, instabilities of the differential rotation are expected to play an important role in determining the angular velocity law. The study of these instabilities is a complex subject (cf. Strittmatter, 1969; Spiegel and Zahn, 1970; Fricke and Kippenhahn, 1970; Zahn, 1974) and here only one will be considered, namely a shear instability (cf. Zahn, 1975) which arises for large values of the Reynolds number. If $\Delta \Omega$ is the latitudinal differential rotation $\left(\Delta \Omega=\Omega_{\mathrm{eq}}-\Omega_{\mathrm{pole}}\right.$ for a given $r$ ), then the Reynolds number corresponding to this differential rotation is defined by $\mathscr{R}_{e}=r^{2} \Delta \Omega / \nu$. For $\Delta \Omega \sim 0.1 \Omega_{0}$ and for typical values of $\nu$ in the radiative region, $\mathscr{R}_{e}$ is very large, and even a weak differential rotation should be unstable. It is instructive to compare the growth rates of these instabilities (cf. Zahn, 1975) with the slowing down time of the Sun as given by Equation (4.7); $\tau_{s}=2 t$. Let $L$ be the smallest scale which is unstable in a differential rotation $\Delta \Omega$, then $L^{2} \Delta \Omega / \nu=\mathscr{R}_{e}^{\text {crit }} \sim$ $10^{3}$. The instability will grow with an $e$-folding time, $L^{2} / \nu, \sim 500 \mathrm{yr}$ (much smaller than $2 t$ ) if $\Delta \Omega=10^{-7} \mathrm{rad} \mathrm{s}^{-1}$.

The penetration of the differential rotation into the radiative region can be visualized as follows: shear instabilities tend to smooth out $\mathrm{d} \Omega / \mathrm{d} \theta$, giving rise to unbalanced pressure forces which drive meridional motions; $\Delta \Omega$ will decrease 
inward, and at a certain $r\left(r_{1}\right), \Delta \Omega$ will become negligible. It is unknown at present if this $r_{1}$ is small enough to explain the Li burning.

If, instead of a shear instability, the Golreich-Schubert and Fricke instability is important, then the radiative region would tend to rotate in cylinders but with the angular velocity increasing inwards. Again instabilities will occur in the upper part of the radiative region because of the different rotation laws in this region and in the convection zone where rotation could be constant along cylinders but with the angular velocity decreasing inwards.

If the solar angular velocity is not uniform (and in particular if no magnetic fields are present) the solar spin-down problem is of great complexity. The most sophisticated treatment at present is that of Sakurai (1975). The reader is referred to this paper and to the review article by Benton and Clark (1974) for further references on this subject.

\section{Discussion}

It is clear that we are not yet certain of the real origin of the Sun's differential rotation; in fact we do not even know whether it can be completely explained within the framework of the equations commonly used in this subject: assume, for example, that differential rotation is generated as a way for the convection zone to minimize the constraints imposed by rotation on convection; this could be achieved by variations in depth and latitude of certain physical parameters (e.g., the turbulent thermal diffusivity and viscosity) that are taken as constants. In fact, it is difficult to avoid the feeling that some of the theories of differential rotation considered in this paper suffer from this limitation and that the action of rotation on the turbulence cannot be ignored. It would be very surprising, however, if the basic mechanism giving rise to differential rotation were to be found entirely outside the theories discussed in this paper. Considerable importance has been given here to theories of differential rotation based on the interaction of rotation with convection. These theories were discussed on the assumption that the solar convection consists mainly of a turbulent convection and a large-scale convection. The dynamics of the convection zone could, of course, be far more complex than this.

On observation in particular, namely, the smallness of the pole-equator difference in flux, $\Delta \mathscr{F}$, has placed very severe restrictions on theories of differential rotation. It is readily seen that this serious difficulty is not necessarily associated with the use of the Boussinesq approximation: even assuming that the perturbations of the pressure, density, and temperature introduced by differential rotation or a meridional circulation are small in relation to the unperturbed values of $p, \rho$ and $T$, the perturbation of the superadiabatic gradient, $\nabla \Delta T$, can be large since $\nabla \Delta T$ is very small itself, in the lower part of the convection zone. It appears, therefore, that a deep penetration of the differential rotation inside the convection zone would imply a major perturbation in the thermodynamics of the convection zone.

Three problems emerge as being of special importance for a further understanding of the Sun's differential rotation:

(1) In the lower part of the convection zone turbulent convection interacts with rotation. An estimate of the magnitude of the latitudinal and radial dependence of this interaction would be of great interest. 
(2) The strength and structure of the large-scale convection in a compressible medium are unknown. In other words, how much heat flux does the large-scale convection carry and how do the magnitudes of the velocities change with depth? Because of their large scale, the action of rotation on these convective motions will be especially important.

(3) Dynamo theories of the solar cycle suggest that differential rotation is not a surface phenomenon. At present it is not understood how large pole-equator differences in the angular velocity $(\Delta \Omega)$ can coexist in the lower part of the convection zone with small pole-equator differences in flux $(\Delta \mathscr{F})$. Of great interest would be an estimate of the typical value of $\Delta \mathscr{F}\left(\right.$ at $\left.r \sim R_{c}\right)$ that can be 'wiped out' in the convection zone $\left(\Delta \mathscr{F}\right.$ very small at $\left.r=R_{0}\right)$. However, even if significant values of $\Delta \mathscr{F}$ inside the convection zone could disappear towards the surface, this would not necessarily mean that these $\Delta \mathscr{F}$ 's are present in the Sun. It is here that the lack of observations of what happens inside the convection zone is particularly felt: it is possible that theories of differential rotation could be developed which agree with the present observations at the solar surface, but which predict significant $\Delta \mathscr{F}$ 's inside the convection zone. It is also conceivable that the convection zone is able to minimize the constraints imposed by rotation on convection in a way that we do not understand at present and that the main effect of this constraint is the generation of a differential rotation with small $\Delta \mathscr{F}$ 's everywhere.

Other points where understanding is lacking and particularly needed have already been discussed in the text; some of them will be summarized here also.

(a) It appears that a deep penetration of differential rotation below the solar surface would entail a major perturbation of the convection zone. Can differential rotation be large only in the upper part and negligible in the lower part of the convection zone? This would have important consequences for theories of the solar dynamo. In this context it would be of interest to know the dependence of $\Omega$ and $\Delta \mathscr{F}$ with depth if the momentum and energy equations are solved with a very small $\Delta \mathscr{F}$ and $\Delta T$ at $r=R_{0}$ and the observed value of $\omega_{2}(r)(=-0.189)$ at the surface.

(b) If differential rotation penetrates deeply into the convection zone, then it must also penetrate into the radiative region; it is now known whether this could be of importance in theories of Lithium depletion in solar-type stars. The influence that a rapidly rotating radiative core (with a period of a week, for example) could have on the conditions at the base of the convection zone is not known either.

\section{Appendix I}

In a system of coordinates rotating with an angular velocity $\Omega_{0}$, the basic equations can be written

$$
\begin{aligned}
& \frac{1}{\sigma} \frac{\partial}{\partial t} \nabla \times \mathbf{U}-\nabla \times \nabla^{2} \mathbf{U}-\mathscr{R}_{1} \nabla \times g(r) T \hat{r}-\mathfrak{T}_{1}(\hat{\omega} \cdot \nabla) \mathbf{U}=-\frac{1}{\sigma} \nabla \times(\mathbf{U} \cdot \nabla) \mathbf{U}, \\
& \left(\frac{\partial}{\partial \mathrm{t}}-\nabla^{2}\right) T=-\mathbf{U} \cdot \nabla T \\
& \operatorname{div} \mathbf{U}=0
\end{aligned}
$$


where $\mathbf{U}, T$, and $g(r)$ are dimensionless variables defined by Equation (2.1.1), $\hat{\omega}$ is a unit vector in the direction of the axis of rotation, and $\mathscr{R}_{1}, \mathfrak{T}_{1}$, and $\sigma$ have been defined following Equations (2.1.5)-(2.1.8).

We expand the velocity field in basic poloidal and toroidal vectors:

$$
\mathbf{U}=\sum_{L, m}\left\{\mathbf{P}\left[p_{L}^{m}(r, t) Y_{L}^{m}(\theta, \phi)\right]+\mathfrak{I}\left[t_{L}^{m}(r, t) Y_{L}^{m}(\theta, \phi)\right]\right\}
$$

In spherical coordinates, the components of $\mathbf{P}\left(p_{L}^{m} Y_{L}^{m}\right)$ and $\mathfrak{T}\left(t_{L}^{m} Y_{L}^{m}\right)$ are given by

$$
\begin{aligned}
& P^{(r)}=\frac{(L+1) L}{r^{2}} p_{L}^{m} Y_{L}^{m}, \quad P^{(\theta)}=\frac{1}{r} \frac{\partial p_{L}^{m}}{\partial r} \frac{\partial Y_{L}^{m}}{\partial \theta}, \\
& P^{(\phi)}=\frac{1}{r \sin \theta} \frac{\partial p_{L}^{m}}{\partial r} \frac{\partial Y_{L}^{m}}{\partial \phi}
\end{aligned}
$$

and

$$
\mathfrak{T}^{(r)}=0, \quad \mathfrak{T}^{(\theta)}=\frac{t_{L}^{m}}{r \sin \theta} \frac{\partial Y_{L}^{m}}{\partial \phi}, \quad \mathfrak{T}^{(\phi)}=-\frac{t_{L}^{m}}{r} \frac{\partial Y_{L}^{m}}{\partial \theta} .
$$

The poloidal and toroidal vectors defined by Equation (A3) form a complete orthogonal set for solenoidal vector fields; $p_{L}^{m}(r, t)$ and $t_{L}^{m}(r, t)$ are the scalars which, together with the spherical harmonic $Y_{L}^{m}(\theta, \phi)$, define a basic poloidal and toroidal vector (Chandrasekhar, 1961, Appendix III).

The dimensionless temperature has been defined in Equation (2.1.3); $\Theta(r, \theta, \phi, t)$ is expanded in spherical harmonics.

$$
\Theta(r, \theta, \phi, t)=\sum_{L, m} \Theta_{L}^{m}(r, t) Y_{L}^{m}(\theta, \phi) .
$$

The spherical harmonics $Y_{L}^{m}(\theta, \phi)$ appearing in Equations (A3) and (A4) are assumed to be normalized according to Condon and Shortley (1951). To obtain the equations for $p_{L}^{m}, t_{L}^{m}, \Theta_{L}^{m}$, and $\psi$ we substitute expression (A2) for $\mathbf{U}$, and expressions (2.1.3) and (A4) for $T$ into Equations (A1), multiply these equations by unit poloidal and toroidal vectors and integrate over the angle coordinates. Neglecting the fluctuating self-interactions (i.e. the right-hand side of Equations (A1)), we obtain (Durney, 1970)

$$
\begin{aligned}
\left(\frac{\partial}{\partial t}-\frac{d^{2}}{\mathrm{~d} r^{2}}-\frac{2}{r} \frac{d}{\mathrm{~d} r}\right) \psi=-\frac{1}{4 \pi r^{2}} \sum_{L, m}^{|m| \leq L}(L+1) L \frac{\partial}{\partial r}\left(r P_{L}^{m} \Theta_{L}^{* m}\right), \\
\left(\frac{\partial}{\partial t}-\mathscr{D}_{L}\right) \Theta_{L}^{m}=\frac{(L+1) L}{r} P_{L}^{m}\left[\frac{\eta}{(1-\eta) r^{2}}-\frac{\partial \psi}{\partial r}\right] \\
\frac{1}{\sigma} \frac{\partial}{\partial t} \mathscr{D}_{L} P_{L}^{m}-\frac{i m}{(L+1) L} \mathfrak{T}_{1} \mathscr{D}_{L} P_{L}^{m}-\mathscr{D}_{L}^{2} P_{L}^{m} \\
=-\mathscr{R}_{1} g_{1}(r) \Theta_{L}^{m}+\mathfrak{I}_{1}\left[L^{-1} A(L, m)(L-1)(L-2) T_{L-1}^{m}\right. \\
\quad-(L+1)^{-1} A(L+1, m)(L+2)(L+3) T_{L+1}^{m} \\
-L^{-1} A(L, m)(L-1) r T_{L-1}^{\prime m}-(L+1)^{-1} A(L+1, m) \\
\left.\quad \cdot(L+2) r T_{L+1}^{\prime m}\right],
\end{aligned}
$$




$$
\begin{aligned}
& \frac{1}{\sigma} \frac{\partial}{\partial t} T_{L}^{m}-\frac{i m}{(L+1) L} \mathfrak{T}_{1} T_{L}^{m}-D_{L} T_{L}^{m} \\
&=-\mathfrak{T}_{1}\left[L^{-1} r^{-2} A(L, m)(L-1)^{2} P_{L-1}^{m}-(L+1)^{-1} r^{-2} A(L+1, m)\right. \\
& \cdot(L+2)^{2} P_{L+1}^{m} \\
&-(r L)^{-1} A(L, m)(L-1) P_{L-1}^{\prime m}-[r(L+1)]^{-1} A(L+1, m) \\
&\left.\cdot(L+2) P_{L+1}^{\prime m}\right],
\end{aligned}
$$

where

$$
\begin{aligned}
& \mathscr{D}_{L}=\frac{d^{2}}{\mathrm{~d} r^{2}}+\frac{2}{r} \frac{d}{\mathrm{~d} r}-\frac{(L+1) L}{r^{2}} ; \quad D_{L}=\frac{d^{2}}{\mathrm{~d} r^{2}}+\frac{4}{r} \frac{d}{\mathrm{~d} r}+\frac{2-(L+1) L}{r^{2}} ; \\
& P_{L}^{m}=p_{L}^{m} / r ; \quad T_{L}^{m}=t_{L}^{m} / r^{2} ; \quad T_{L}^{\prime m}=\frac{\mathrm{d} T_{L}^{m}}{\mathrm{~d} r} ; \quad P_{L}^{\prime m}=\frac{\mathrm{d} P_{L}^{m}}{\mathrm{~d} r} \\
& A(L, m)=[(L+m)(L-m) /(2 L+1)(2 L-1)]^{1 / 2} .
\end{aligned}
$$

The asterisk (cf. Equation (A5a)) defines the complex conjugate of $\Theta_{L}^{m}, \eta\left(=R_{c} / R_{0}\right)$ is the ratio between the inner and outer radius of the spherical shell and $g_{1}(r)=$ $g(r) / r$. Free-surface boundary conditions at $r=1$, for example, imply $P_{L}^{\prime m}=T_{L}^{\prime m}=0$ $(r=1)$. The spherical harmonics, as defined by Condon and Shortley (1951), satisfy the following relation

$$
Y_{L}^{m}(\theta, \phi)=(-1)^{m} Y_{L}^{m^{*}}(\theta, \phi)
$$

From the expansions (A2) and (A4) it follows that the velocity and temperature fields will be real if

$$
P_{L}^{-m}=(-1)^{m} P_{L}^{m^{*}} ; \quad T_{L}^{-m}=(-1)^{m} T_{L}^{m^{*}} ; \quad \Theta_{L}^{-m}=(-1)^{m} \Theta_{L}^{m^{*}}
$$

It is readily seen that these relations are a consequence of Equations (A5).

If Equations (A5) are integrated in time, the quantities $P_{L}^{m}, T_{L}^{m}, \Theta_{L}^{m}$, and $\psi$ which determine the velocity and temperature field do not grow indefinitely. This is due to the non-linear term, $-[(L+1) L / r] P_{L}^{m}(\partial \psi / \partial r)$, of Equation (A5b): the interaction of the velocity field with $\partial \psi / \partial r$ (the distortion in the mean temperature gradient produced by convection) stabilizes the fluctuating component of the temperature field, $\Theta_{L}^{m}$. In relation to its great simplicity, the mean-field approximation can be considered to be very successful (cf. Herring, 1963, 1964, 1969; Durney, 1968a).

In Equations (A5) we have neglected the fluctuating self-interactions. The expressions of $\mathbf{U} \cdot \nabla \mathbf{U}$ and $\mathbf{U} \cdot \nabla \Theta$ in terms of the defining scalars of the velocity and temperature fields are complex, but they have now been worked out by Young (1974).

For $m \neq 0$, the solutions of Equations (A5) are time-dependent (cf. Busse, 1970, 1973; Durney, 1970; Yoshimura and Kato, 1971). This is a general property of non-radial motions of rotating spheres or spherical shells (cf., for example, Durney and Skumarich, 1968, for the case of marginally unstable, non-radial oscillations of a polytrope). If we multiply Equation (A5c) by $\mathscr{D}_{L}$, use Equation (A5b) to express $-\mathscr{R}_{1} g_{1} \mathscr{D}_{L} \Theta_{L}^{m}$ in terms of $\mathscr{R}_{1} g_{1} \times \mathrm{RHS}(\mathrm{A} 5 \mathrm{~b})$ and $-i \omega \mathscr{R}_{1} g_{1} \Theta_{L}^{m}(\partial / \partial t=i \omega)$, and finally replace $-\mathscr{R}_{1} g_{1} \Theta_{L}^{m}$, in this last expression, by $-\mathscr{D}_{L}^{2} P_{L}^{m}$ (note that Equation (A5c) 
reduces to $\mathscr{R}_{1} g_{1} \Theta_{L}^{m}=\mathscr{D}_{L}^{2} P_{L}^{m}$ in the case of no rotation), we obtain

$$
\begin{gathered}
\frac{i}{\sigma}\left(\omega(\sigma+1)-\frac{m \sigma}{(L+1) L} \mathfrak{T}_{1}\right) \mathscr{D}_{L}^{2} p_{L}^{m}-\mathscr{D}_{L}^{3} P_{L}^{m} \\
=\mathscr{R}_{1} g(r) \times \operatorname{RHS}(\mathrm{A} 5 \mathrm{~b})+0\left(\mathfrak{I}_{1}^{2}\right) .
\end{gathered}
$$

The first term in this equation vanishes if $\omega_{d}=2 m \Omega_{0} /(L+1) L(1+\sigma)$, which is Equation (3.8) of Busse (1970); $\omega_{d}$ in this last expression is the dimensional value of $\omega$. (The reader is referred to Busse, 1973 and Heard and Veronis, 1973 for consistent solutions of the Boussinesq equations for convection, in the case of small rotation, and small convective amplitudes.) For $m=L=10$, corresponding to the most unstable mode (see below) of a rotating shell of thickness $d=0.2 R_{0}$, the value of $\omega_{d}$ equals $\Omega_{0} / 11$ (for $\sigma=1$ ). These convective waves, which propagate in the opposite sense of rotation (i.e., are retrograde) for small Taylor numbers, become prograde for large values of $\mathfrak{I}_{1}$ (Gilman, 1975, Figures 4 and 5).

For values of $\mathscr{R}_{1}$ and $\mathfrak{T}_{1}$ that are not very large, the values of $L$ and $m$ corresponding to the most unstable modes are mainly determined, respectively, by the thickness of the spherical shell ( $L=10$ for $\eta=0.8$; Durney, 1968a) and by rotation $(m=L)$ (Busse, 1970; Durney, 1970; Yoshimura and Kato, 1971). The reader is referred to Figures 1 and 2 of Gilman (1975) for a plot (for different values of $\mathfrak{I}_{1}$ ) of the critical Rayleigh number versus $m$.

According to Equations (A5), rotation couples the different values of $L$ in the form $\mathbf{P}_{\mathbf{L}}^{L}-T_{L+1}^{L}-P_{L+2}^{L}-T_{L+3}^{L}-\cdots=M_{L}^{L}$. In the presence of rotation this coupling scheme defines the mode $M_{L}^{L}\left(P_{L}^{L}\right.$ has been written in bold face to indicate that $P_{L}^{L}$ is the largest poloidal mode). The above statement that $m=L$ is the most unstable mode means, therefore, that the mode $M_{L+2}^{L}=P_{L}^{L}-T_{L+1}^{L}-\mathbf{P}_{\mathbf{L}+2}^{\mathbf{L}}-T_{L+3}^{L} \cdots$, for example, has a larger critical Rayleigh number than the mode $M_{L}^{L}$. The fluctuating self-interactions couple modes with different values of $m$, as well as $L$ (cf. Busse, 1973; Heard and Veronis, 1973; Gilman, 1975).

\section{Appendix II}

We intend here to estimate, in an approximate way, the right-hand side of Equations (2.1.6) and (2.1.8) to gain a very crude understanding of the relative magnitude of the pole-equator difference in flux and latitudinal differential rotation.

The dimensionless values of $r$ will be assumed to be of the order unity, numerical factors will generally be ignored, and one will be neglected with respect to $L$. We define $K_{L}^{m}(\theta)$ by $Y_{L}^{m}=(1 / \sqrt{2 \pi}) e^{i m \phi} K_{L}^{m}(\theta)$, and perform the calculations for the mode $M_{L}^{m}$. Equation (A3a) then gives, for the convective velocities:

$$
\begin{aligned}
& U_{r}^{c} \sim L^{2} P_{L}^{m}(r) K_{L}^{m}(\theta) \cos m \phi \\
& U_{\theta}^{c} \sim P_{L}^{m}(r) \frac{\partial K_{L}^{m}(\theta)}{\partial \theta} \cos m \phi / \xi \\
& U_{\phi}^{c} \sim P_{L}^{m}(r) m K_{L}^{m}(\theta) \sin m \phi / \xi \sin \theta
\end{aligned}
$$

where $\xi=1-\eta$ is the dimensionless thickness of the spherical shell, and $\mathrm{d} P_{L}^{m} / \mathrm{d} r$ has 
been set equal to $P_{L}^{m} / \xi$. The thinner the spherical shell is, the larger becomes the value of $L$ of the most unstable mode, i.e., $\xi L \sim 1$. In what follows, we assume that the values of $\xi$ and $L$ are related in this way.

An estimate of $T_{L+1}^{m}$ can be obtained from Equation (A5d), which we simplify to

$$
D_{L+1} T_{L+1}^{m} \sim\left(d^{2} / \mathrm{d} r^{2}-L^{2} / r^{2}\right) T_{L+1}^{m} \sim \mathfrak{T}_{1} L A(L+1, m) P_{L}^{m} .
$$

Therefore, $T_{L+1}^{m} \sim \mathfrak{T}_{1} P_{L}^{m} A(L+1, m) / L$. (The equation $\left(d^{2} / \mathrm{d} r^{2}-L^{2} / r^{2}\right) T_{L+1}^{m}=$ $\sin (\pi r / \xi)$ can be solved by Green's functions and it is readily seen that $\left.T_{L+1}^{m} \sim 1 / L^{2}\right)$. With the help of Equation (A3b) we obtain, for the toroidal velocities,

$$
\begin{aligned}
U_{\theta}^{\Omega} & \sim T_{L+1}^{m} m \sin m \phi K_{L+1}^{m}(\theta) / \sin \theta \\
& \sim m P_{L}^{m} \mathfrak{T}_{1} A(L+1, m) \sin m \phi K_{L+1}^{m}(\theta) / L \sin \theta \\
U_{\phi}^{\Omega} & \sim P_{L}^{m} \mathfrak{T}_{1} A(L+1, m) \cos m \phi\left(\partial K_{L+1}^{m} / \partial \theta\right) / L .
\end{aligned}
$$

The integrals appearing in the right-hand side of Equation (2.1.6) are

$$
\begin{aligned}
& I_{1}=\frac{\partial}{\partial r} \int \sin ^{2} \theta r^{3}\left\langle U_{\phi}^{\Omega} U_{r}^{c}\right\rangle \mathrm{d} \theta ; \\
& I_{2}=\int \cos \theta \sin \theta\left\langle U_{\theta}^{c} U_{\phi}^{\Omega}\right\rangle \mathrm{d} \theta ; \\
& I_{3}=\int \cos \theta \sin \theta\left\langle U_{\phi}^{c} U_{\theta}^{\Omega}\right\rangle \mathrm{d} \theta .
\end{aligned}
$$

(a) $\left\langle U_{r}^{c} U_{\phi}^{\Omega}\right\rangle=L \mathfrak{I}_{1}\left(P_{L}^{m}\right)^{2} A(L+1, m) K_{L}^{m}(\theta) \partial K_{L+1}^{m} / \partial \theta$. With the help of Equation (19) of Condon and Shortley (1951) (CS), we can express $\partial K_{L+1}^{m} / \partial \theta$ in terms of $K_{L+1}^{m+1}(\theta)$; and with the help of their formula (21) we can express $\sin \theta K_{L}^{m}(\theta)$ in terms of $K_{L+1}^{m+1}(\theta)$. We obtain for the integral $I_{1}$,

$$
I_{1} \sim L^{2} \mathfrak{T}_{1}\left(P_{L}^{m} A(L+1, m)\right)^{2} / \xi
$$

(b) $\left\langle U_{\theta}^{c} U_{\phi}^{\Omega}\right\rangle \sim \mathfrak{I}_{1} A(L+1, m)\left(P_{L}^{m}\right)^{2}\left(\partial K_{L}^{m} / \partial \theta\right)\left(\partial K_{L+1}^{m} / \partial \theta\right) / \xi L$. with the help of Equations (19) and (21) of CS, we express $\partial K_{L}^{m} / \partial \theta$ and $\partial K_{L+1}^{m} / \partial \theta$ in terms of $K_{L}^{m-1}$ and $K_{L+1}^{m-1}$, respectively, and $\cos \theta K_{L}^{m-1}$ in terms of $K_{L+1}^{m-1}$. We obtain for $I_{2}$

$$
I_{2} \sim \mathfrak{I}_{1}(L-m+2)\left(P_{L}^{m} A(L+1, m)\right)^{2} / \xi
$$

(c) $\left\langle U_{\theta}^{\Omega} U_{\phi}^{c}\right\rangle \sim m^{2} \mathfrak{T}_{1}\left(P_{L}^{m}\right)^{2} A(L+1, m) K_{L+1}^{m}(\theta) \quad K_{L}^{m}(\theta) / \xi L \sin ^{2} \theta$; we express $\cos \theta K_{L+1}^{m}(\theta)$ in terms of $K_{L}^{m}(\theta)$ (cf. Equation (21) of CS) and obtain

$$
I_{3} \sim m^{2} \mathfrak{I}_{1}\left(P_{L}^{m} A(L+1, m)\right)^{2} / \xi L
$$

We discuss now whether the angular momentum transport given by the integrals $I_{1}, I_{2}$, and $I_{3}$ accelerates or decelerates the equatorial regions.

(a') If $m=L$, then $U_{r}^{c} \sim L^{2} P_{L} \sin ^{L} \theta \cos L \phi$ and $U_{\theta}^{c} \sim L\left(\mathrm{~d} P_{L} / \mathrm{d} r\right) \sin ^{L-1} \theta$ $\cos \theta \cos L \phi$. Near the outer surface $\left|U_{\theta}^{c}\right| \gg\left|U_{r}^{c}\right|$, since $U_{r}^{c}=0$ for $r=R_{0}$. This inequality fails to be satisfied only for values of $\theta$ very close to $\pi / 2$. Also if, for example, $U_{r}^{c}>0$, then $U_{\theta}^{c}<0(>0)$ for $\theta<\pi / 2(>\pi / 2)$. It is readily seen that in both cases the action of Coriolis forces on the convective velocities (which, with the exception of a small region around the equator, are mainly latitudinal near the outer 
surface) generates a $U_{\phi}^{\Omega}$ which is positive. Therefore, with the exception of the above region, the term $\left\langle U_{r}^{c} U_{\phi}^{\Omega}\right\rangle$ transports angular momentum outwards (for $r \sim R_{0}$ ), since an upgoing particle carries a $U_{\phi}^{\Omega}>0\left(\left\langle U_{r}^{c} U_{\phi}^{\Omega}\right\rangle>0\right)$. At the equator and just below the surface, $\left\langle U_{r}^{c} U_{\phi}^{\Omega}\right\rangle$ is small in absolute value, and negative (since for $\theta=\pi / 2, U_{\theta}^{c}=0$, and a radially upgoing particle acquires a negative $\left.U_{\phi}^{\Omega}\right)$. At a small latitude, $\left\langle U_{r}^{c} U_{\phi}^{\Omega}\right\rangle$ becomes positive, reaches a maximum at, say, $\theta=\theta_{m}$ and vanishes at the poles. Therefore, if the vertical transport of angular momentum is important, one would expect the surface angular velocity to be maximum at $\theta \sim \theta_{m}$ and not at the equator (cf. Gilman, 1972, Figure 10).

The contribution of $\left\langle U_{\phi}^{\Omega} U_{r}^{c}\right\rangle$ to $T_{3}(r)$ can be understood as follows: we approximate Equation (2.1.6) by $-10 T_{3}(r) / r^{2}=\operatorname{RHS}(2.1 .6)\left(\mathrm{d} T_{3}(r) / \mathrm{d} r=0\right.$ at $\left.r=R_{0}\right)$. The equator rotates faster than the poles if $T_{3}(r)<0$ (cf. Equation (2.1.2)). Therefore, positive contributions to RHS (2.1.6) give rise to equatorial acceleration. Equation (2.1.6) shows that if

$$
\int\left(5 \cos ^{2} \theta-1\right) \frac{\partial}{\partial r}\left(r^{3}\left\langle U_{\phi}^{\Omega} U_{r}^{\gamma}\right\rangle\right) \mathrm{d} \theta>0,
$$

then the vertical transport of angular momentum contributes negatively to $T_{3}\left(R_{0}\right)$.

$\left(b^{\prime}\right)$ If $U_{r}^{c}>0$, then in the outer layers $U_{\theta}^{c}<0$ and, as seen above, $U_{\phi}^{\Omega}>0(\theta \sim \pi / 2)$ excepted). Therefore, the term $\left\langle U_{\theta}^{c} U_{\phi}^{\Omega}\right\rangle$ is negative, and this term is expected to give rise to equatorial deceleration $T_{3}\left(R_{0}\right)>0$. From Equation (B2b) it follows that $I_{2}$ is smallest (compared with $I_{1}$ and $I_{3}$ ) when $m=L$.

(c') If $U_{\phi}^{c}>0$, then the Coriolis forces generate a $U_{\theta}^{\Omega}$ that is also positive. The term $\left\langle U_{\theta}^{\Omega} U_{\phi}^{c}\right\rangle$ transports angular momentum towards the equator $\left(I_{3}\right.$ contributes to a negative $\left.T_{3}\left(R_{0}\right)\right)$. From Equation $(\mathrm{B} 2 \mathrm{c})$ this transport of angular momentum is largest if $m=L$. (In the above discussion when we say, for example, that if $U_{\phi}^{c}>0$ then $U_{\theta}^{\Omega}$ is also larger than zero, we neglect viscosity and consider that the particle is acted upon only by the Coriolis force. Nevertheless, we expect the conclusions to give us a correct description.)

It is readily seen (cf. Equations (2.1.1) and (2.1.2) that to obtain the observed differential rotation $\left(\Omega_{\text {equator }}-\Omega_{\text {pole }} \sim \Omega_{0} / 5\right)$ the value of $T_{3}\left(R_{0}\right)$ must be given by

$$
T_{3}\left(R_{0}\right)=-\frac{4 \Omega_{0}}{75}\left(\frac{\pi}{7}\right)^{1 / 2} \frac{R_{0}^{2}}{\kappa} .
$$

If we assume that the main contribution to $T_{3}\left(R_{0}\right)$ comes from the latitudinal angular momentum transport we obtain, with the help of Equations (2.1.6) (with $\xi L \sim 1$ ) and (B2c):

$$
T_{3}=-\frac{(7 \pi)^{1 / 2}}{16 \sigma} L^{2} \mathfrak{T}_{1}\left(P_{L}^{L}\right)^{2} / L
$$

Comparing this equation with Equation (B3), we can estimate the magnitude of the convective velocities needed to generate the observed latitudinal differential rotation:

$$
\left(L P_{L}^{L}\right)^{2} \sim L \sigma^{2} / 16
$$


Since these large-scale convective motions are concentrated near the equator, they give rise to a pole-equator difference in flux. The ratio of the convective flux (at $r=0.9$, for example) to the purely conductive flux (evaluated in the absence of convective motions) is given by

$$
\begin{aligned}
Q & =r^{2} \iint U_{r}^{c} \theta \sin \Theta \mathrm{d} \theta \mathrm{d} \phi /(4 \pi \eta /(1-\eta)) \\
& \sim \frac{1-\eta}{4 \pi} \iint U_{r}^{c} \Theta \sin \theta \mathrm{d} \theta \mathrm{d} \phi
\end{aligned}
$$

An estimate of $\Theta$ for the $(L, L)$ mode $\left(\Theta \sim \Theta_{L}^{L}\right)$ can be obtained from Equation (A5b): $\Theta_{L}^{L} \sim P_{L}^{L}$. This order-of-magnitude relation holds only if $\partial \psi / \partial r \ll \eta /(1-\eta) r^{2}$, that is, if the distortion of the mean temperature gradient, produced by convection, is small (cf. Durney, 1968a, Figures 2 and 3). With the above value of $\Theta_{L}^{L}$, it is readily found that

$$
Q \sim(1-\eta)\left(L P_{L}^{L}\right)^{2} \sim(1-\eta) L \sigma^{2} / 16
$$

For $L=10$ and $\sigma=1, Q \sim 0.12$. The value of $Q$ given by Equation (B5) should not be taken too literally (it is probably an underestimate; see Gilman, 1972, Figure 10). Equation (B5) shows, however, a basic difficulty associated with this approach to differential rotation: to generate the observed solar differential rotation, an appreciable fraction of the energy flux must be carried by the large-scale convection. To give rise to transport of angular momentum towards the equator, this large-scale convection must be highly non-axisymmetric $(m \sim L)$ and must be concentrated, therefore, near the equator. Large pole-equator differences in flux appear unavoidable. It is of interest to note that our estimate of the pole-equator difference in flux $(Q)$ needed to generate the observed differential rotation shows that $Q$ is proportional to the square of the Prandtl number.

\section{Appendix III}

We choose normalized spherical coordinates; it is thus unnecessary to distinguish between covariant and contravariant components of vectors and tensors. In the case of axial symmetry, the components of the viscous force and the viscous stress tensor are given by

$$
\begin{aligned}
& R_{r}=\frac{1}{r^{2}} \frac{\partial}{\partial r}\left(r^{2} T_{r r}\right)+\frac{1}{r \sin \theta} \frac{\partial}{\partial \theta}\left(\sin \theta T_{\theta r}\right)-\frac{T_{\theta \theta}+T_{\phi \phi}}{r} \\
& R_{\theta}=\frac{1}{r^{3}} \frac{\partial}{\partial r}\left(r^{3} T_{r \theta}\right)+\frac{1}{r \sin \theta} \frac{\partial}{\partial \theta}\left(\sin \theta T_{\theta \theta}\right)-\frac{\operatorname{cotg} \theta}{r} T_{\phi \phi} \\
& R_{\phi}=\frac{1}{r^{3}} \frac{\partial}{\partial r}\left(r^{3} T_{\phi r}\right)+\frac{1}{r \sin ^{2} \theta} \frac{\partial}{\partial \theta}\left(\sin ^{2} \theta T_{\theta \phi}\right)
\end{aligned}
$$

with

$$
\begin{aligned}
& T_{r r}=2 s \eta \frac{\partial U_{r}}{\partial r}+2 \eta(1-s) \frac{\partial U_{r}}{\partial r} \\
& T_{r \theta}=\frac{s \eta}{r}\left[\frac{\partial U_{r}}{\partial \theta}+r \frac{\partial U_{\theta}}{\partial r}-U_{\theta}\right]+\frac{\eta(1-s)}{r} \frac{\partial\left(r U_{\theta}\right)}{\partial r}
\end{aligned}
$$




$$
\begin{aligned}
& T_{r \phi}=\sin \theta\left[s \eta r \frac{\partial \Omega}{\partial r}+\frac{\eta(1-s)}{r} \frac{\partial \Omega r^{2}}{\partial r}\right] \\
& T_{\theta \theta}=\frac{2 \eta s}{r}\left(U_{r}+\frac{\partial U_{\theta}}{\partial \theta}\right) \\
& T_{\theta \phi}=s \eta \sin \theta \frac{\partial \Omega}{\partial \theta} \\
& T_{\phi \phi}=\frac{2 s \eta}{r}\left[U_{r}+U_{\theta} \operatorname{cotg} \theta\right] .
\end{aligned}
$$

An anisotropic turbulent viscosity of the following form has been assumed $\eta_{\theta \theta}=$ $\eta_{\phi \phi}=s \eta_{r r}=s \eta$.

\section{Appendix IV}

With $p, \rho, T, U_{r}$ and $U_{\theta}$ defined by Equations (2.3.8), (2.3.9) we obtain from Equations (2.3.6):

$$
\begin{aligned}
\rho_{u} \rho_{2} g= & -\left(p_{u} p_{2}\right)^{\prime}+\nu\left[2 \psi^{\prime \prime} / r^{2}-2 \psi^{\prime} \rho^{\prime} / 3 r^{2} \rho\right. \\
& \left.-\psi\left(12 / r^{4}+8 \rho^{\prime} / 3 r^{3} \rho+8\left(\rho^{\prime} / \rho\right)^{\prime} / 3 r^{2}\right)\right] \\
p_{u} p_{2}= & \frac{\nu}{3}\left[\psi^{\prime \prime \prime}-\psi^{\prime \prime} \rho^{\prime} / \rho-\psi^{\prime}\left(6 / r^{2}+2 \rho^{\prime} / r \rho\right.\right. \\
& \left.\left.+\left(\rho^{\prime} / \rho\right)^{\prime}\right)+\psi\left(12 / r^{3}+4 \rho^{\prime} / r^{2} \rho\right)\right] .
\end{aligned}
$$

In these equations the primes denote derivatives with respect to $r$; the viscosity has been assumed to be isotropic and of the form $\eta=\rho \nu$ with $\nu$ (a slowly varying quantity in the Sun) constant.

$$
\begin{aligned}
& -\gamma \psi^{\prime \prime \prime}+\left[\gamma \frac{\rho^{\prime}}{\rho}+\frac{p^{\prime}}{p}\right] \psi^{\prime \prime \prime}+\left[\frac{12 \gamma}{r^{2}}+\frac{2 \gamma}{r} \frac{\rho^{\prime}}{\rho}+2 \gamma\left(\frac{\rho^{\prime}}{\rho}\right)^{\prime}-\frac{p^{\prime}}{p} \frac{\rho^{\prime}}{\rho}\right] \psi^{\prime \prime} \\
& +\left[-\frac{24 \gamma}{r^{3}}-\frac{8 \gamma}{r^{2}} \frac{\rho^{\prime}}{\rho}-\frac{6 p^{\prime}}{r^{2} p}+\frac{2 \gamma}{r}\left(\frac{\rho^{\prime}}{\rho}\right)^{\prime}-\frac{2}{r} \frac{\rho^{\prime}}{\rho} \frac{p^{\prime}}{p}+\gamma\left(\frac{\rho^{\prime}}{\rho}\right)^{\prime \prime}-\left(\frac{\rho^{\prime}}{\rho}\right)^{\prime} \frac{p^{\prime}}{p}\right] \psi^{\prime} \\
& +\left[\frac{12}{r^{3}} \frac{p^{\prime}}{p}-\frac{12 \gamma}{r^{2}}\left(\frac{\rho^{\prime}}{\rho}\right)^{\prime}+\frac{4}{r^{2}} \frac{p^{\prime}}{p} \frac{\rho^{\prime}}{\rho}\right] \psi=-\frac{4(\gamma-1) \Omega_{0} \mathscr{F}_{0} \varepsilon g \rho_{u}}{\nu^{3}} \int_{R_{0}}^{r} \frac{l^{2}}{p_{u}} \mathrm{~d} r .
\end{aligned}
$$

For a polytrope and for $l=-1.5 p_{u} / p_{u}^{\prime}$ the right-hand side of Equation (D3) becomes

$$
\mathrm{RHS}=\left.\frac{9(\gamma-1)^{3} L^{2} \Omega_{0} \mathscr{F}_{0} \varepsilon}{\gamma \nu^{3}(2 \gamma-3)}\left(1-\left(r-R_{c}\right) / L\right)^{2}\right|_{R_{0}} ^{r} .
$$

\section{Appendix V}

The viscosity coefficient is taken to be proportional to the density: $\eta=\rho \nu$, and the anisotropy factor $s$ is defined by $\nu_{\theta \theta}=\nu_{\phi \phi}=s \nu_{r r}=s \nu$. The meridional motions are 
assumed to be given by Equation (2.3.9), and the angular velocity $\Omega$ by Equation (2.3.17). The equations for $\omega_{0}(r)$ and $\omega_{2}(r)$ are then obtained from Equation (2.3.3) (Durney, 1974b):

$$
\begin{aligned}
\omega_{0}^{\prime \prime}+ & \left(\frac{4}{r}+\frac{\rho^{\prime}}{\rho}\right) \omega_{0}^{\prime}-\frac{2 \omega_{2} s}{r^{2}}+\frac{2(1-s)}{r}\left(\omega_{0}^{\prime}+\frac{\rho^{\prime}}{\rho} \omega_{0}+\frac{3 \omega_{0}}{r}\right) \\
& -\frac{2 \psi}{5 r^{3} \rho \nu}\left(2 \omega_{2}+\omega_{2}^{\prime} r\right)+\frac{2}{3} \frac{\psi^{\prime} r}{r^{3} \rho \nu}\left[\omega_{0}-\frac{1}{5} \omega_{2}\right] \\
= & -\frac{2}{3} \frac{\psi^{\prime} r}{r^{3} \rho \nu}-\frac{2(1-s)}{r}\left(\frac{\rho^{\prime}}{\rho}+\frac{3}{r}\right), \\
\omega_{2}^{\prime \prime}+ & \left(\frac{4}{r}+\frac{\rho^{\prime}}{\rho}\right) \omega_{2}^{\prime}-\frac{10 \omega_{2} s}{r^{2}}+\frac{2(1-s)}{r}\left(\omega_{2}^{\prime}+\frac{\rho^{\prime}}{\rho} \omega_{2}+\frac{3 \omega_{2}}{r}\right) \\
& -\frac{2 \psi}{r^{3} \rho \nu}\left[2 \omega_{0}+r \omega_{0}^{\prime}+\frac{2\left(2 \omega_{2}+r \omega_{2}^{\prime}\right)}{7}\right]+\frac{4 \psi^{\prime} r}{3 r^{3} \rho \nu}\left(\omega_{0}+\frac{4 \omega_{2}}{7}\right) \\
= & \frac{4 \psi}{r^{3} \rho \nu}-\frac{4 \psi^{\prime} r}{3 r^{3} \rho \nu} .
\end{aligned}
$$

From the difference $5 \rho r^{4}(\mathrm{E} 1)-\rho r^{4}(\mathrm{E} 2)$ it is easily shown that

$$
\begin{gathered}
\frac{d}{\mathrm{~d} r}\left[5 r^{4} \rho \omega_{0}^{\prime}+10(1-s) r^{3} \rho\left(1+\omega_{0}\right)-r^{4} \rho \omega_{2}^{\prime}-2(1-s) r^{3} \rho \omega_{2}\right. \\
\left.-\frac{10}{7 \nu} \psi r^{2} \omega_{2}+\frac{2}{\nu} \psi r^{2} \omega_{0}+\frac{2}{\nu} \psi r^{2}\right]=0
\end{gathered}
$$

Equation (E3) is a particular form of Equation (E4):

$$
\frac{d}{\mathrm{~d} r} \iint r^{3} \sin ^{2} \theta\left(T_{\phi r}-\rho U_{r} U_{\phi}\right) \mathrm{d} \theta \mathrm{d} \phi=0 .
$$

Equation (E4) can be derived from the steady state azimuthal equation of motion (see Equation (2.3.3)) with only one assumption: the dependence of $\rho$ on $\theta$ is small and can be neglected; in particular, the assumption of axial symmetry is unnecessary.

The boundary conditions that are commonly imposed on Equations (E1, E2) are zero stresses and vertical velocities $\left(T_{\phi r}=U_{r}=0\right)$ at both ends of the convection zone $\left(r=R_{c}, R_{0}\right)$. In this case the integrated version of Equation (E4) is the compressible version of Equation (2.1.9). It should be noted that in the compressible case we have expanded the angular velocity in Legendre polynomials whereas in the Boussinesq approximation the expansion of the toroidal velocities is given by Equation (A3b) of Appendix I: it is an expansion in terms of $\partial P_{L}(\cos \theta) / \partial \theta$.

\section{Appendix VI}

The perturbed pressure, density, and temperature are expanded in Legendre polynomials according to Equation (2.4.8). We neglect quadratic terms in the 
differential rotation (i.e., $\omega_{0}^{2}, \omega_{2}^{2}, \omega_{0} \omega_{2}$ ) and in the perturbation (i.e., $\chi_{i} \xi_{j}$; where $\chi, \xi$ stand for $p, \rho, T$ and $i, j=0,2,4)$. Then

$$
\begin{aligned}
& U_{\phi}^{2} / r=\frac{2}{3} \Omega_{0}^{2} r\left[1+2 \omega_{0}-2 \omega_{2} / 5-P_{2}\left(1+2 \omega_{0}-10 \omega_{2} / 7\right)-36 \omega_{2} P_{4} / 35\right] \\
& U_{\phi}^{2} \operatorname{cotg} \theta / r=\Omega_{0}^{2} r\left(1+2 \omega_{0}+2 \omega_{2} P_{2}\right) \sin \theta \cos \theta .
\end{aligned}
$$

Substituting Equations (2.4.8) and (F1, F2) into Equations (2.4.1) and (2.4.2), we obtain

$$
\begin{aligned}
\mathrm{d} p_{u} / \mathrm{d} r & =-\rho_{u} g ; \quad \mathrm{d} p_{0} / \mathrm{d} r=-\rho_{0} g+\frac{2}{3} \Omega_{0}^{2} r \rho_{u}\left(1+2 \omega_{0}-2 \omega_{2} / 5\right) ; \\
\mathrm{d} p_{2} / \mathrm{d} r & =-\rho_{2} g-\frac{2}{3} \Omega_{0}^{2} r \rho_{u}\left(1+2 \omega_{0}-10 \omega_{2} / 7\right) ; \\
\mathrm{d} p_{4} / \mathrm{d} r & =-\rho_{4} g-\frac{24}{35} \Omega_{0}^{2} r \rho_{u} \omega_{2} . \\
p_{2} & =-\frac{1}{3} \rho_{u} \Omega_{0}^{2} r^{2}\left(1+2 \omega_{0}+2 \omega_{2} / 7\right) ; \\
p_{4} & =-\frac{6}{35} \Omega_{0}^{2} r^{2} \rho_{u} \omega_{2} .
\end{aligned}
$$

With the help of the gas equation $\left[p_{i}=(R / \mu)\left(\rho_{i} T_{u}+\rho_{u} T_{i}\right), i=2,4\right]$, Equations (F3) allow us to calculate $T_{2}$ and $T_{4}$; we obtain

$$
\begin{aligned}
T_{2}= & -\frac{1}{3} \Omega_{0}^{2} r^{2}\left(\mu / R+\rho_{u}^{\prime} T_{u} / g \rho_{u}\right)\left(1+2 \omega_{0}+2 \omega_{2} / 7\right) \\
& -\frac{2}{21} \Omega_{0}^{2} \frac{r T_{u}}{g}\left(7 r \omega_{0}^{\prime}+r \omega_{2}^{\prime}+12 \omega_{2}\right) \\
T_{4}= & -\frac{6}{35} \Omega_{0}^{2} r^{2} \omega_{2}\left(\mu / R+\rho_{u}^{\prime} T_{u} / g \rho_{u}\right)-\frac{6}{35} \frac{\Omega_{0}^{2} r T_{u}}{g}\left(r \omega_{2}^{\prime}-2 \omega_{2}\right) .
\end{aligned}
$$

We assume that the angular velocity and meridional motions are given by Equations (2.4.14) and (2.3.7), respectively. Keeping only the largest term of $R_{\phi}\left(\nu \rho_{u}^{\prime} r \sin \theta \partial \Omega / \partial r\right)$ but all inertial terms of Equation (2.3.3), it is readily found that the azimuthal equation of motion can be written

$$
\begin{aligned}
\frac{\partial \psi_{1}}{\partial \theta} \sin \theta-\frac{\partial \psi_{1}}{\partial r} r \cos \theta & \\
= & \frac{3 \omega_{2}\left(R_{c}\right) \nu \rho_{u}^{\prime}(r \sin \theta)^{4}}{2 R_{c}^{2}\left(1-\frac{3}{2} \omega_{2}\left(R_{c}\right)\left(r / R_{c}\right)^{2} \sin ^{2} \theta\right)} .
\end{aligned}
$$

Equation (F5) can be solved analytically if the pressure and density are related by a polytropic relation $\left(p_{u}=p_{u c}\left(\rho / \rho_{u c}\right)^{\gamma}\right)$ with $\gamma=\frac{3}{2}$, which is very close to the adiabatic $\left(\gamma=\frac{5}{3}\right)$ value (we neglect perturbations in pressure and density due to the motions). The general solution of Equation (F5) is in this case given by

$$
\begin{aligned}
\psi_{1}= & -\frac{3 \omega_{2}\left(R_{c}\right) \nu \rho_{c}(r \sin \theta)^{4}}{\left(R_{c} L\right)^{2}\left(1-\frac{3}{2} \omega_{2}\left(R_{c}\right)\left(r \sin \theta / R_{c}\right)^{2}\right)} \\
& \times\left[\left(L+R_{c}\right) \log \frac{1+\cos \theta}{\sin \theta}-r \cos \theta\right]+\xi(r \sin \theta)
\end{aligned}
$$

where $L$ has been defined in Equation (2.3.15) and $\xi(r \sin \theta)$ is an arbitrary function of $r \sin \theta$. 


\section{References}

Abt, H. and Hunter, J.: 1962, Astrophys. J. 136, 381.

Alfonso-Faus, A.: 1967, J. Geophys. Res. 72, 5576.

Altrock, R. C. and Canfield, R. C.: 1972a, Astrophys. J. 171, L71.

Altrock, R. C. and Canfield, R. C.: 1972b, Solar Phys. 23, 257.

Antonucci, E. and Svalgaard, L.: 1974, Solar Phys. 34, 3.

Appenzeller, I. and Schröter, E. H.: 1967, Astrophys. J. 147, 1100.

Appenzeller, I. and Schröter, E. H.: 1968, Solar Phys. 4, 131.

Baker, N. and Temesvary, S.: 1966, Tables of Convective Stellar Envelope Models, 2nd edn., Goddard Institute for Space Studies, New York.

Beckers, J. M. and Morrison, R. A.: 1970, Solar Phys. 14, 280.

Belvedere, G. and Paterno, L.: 1976, Solar Phys. (in press).

Benton, E. R. and Clark, A.: 1974, Ann. Rev. Fluid Mech. 6, 257.

Biermann, L.: 1951, Z. Astrophys. 28, 304.

Böhm, K. H.: 1963, Astrophys. J. 137, 881.

Boussinesq, J.: 1877, 'Essai sur la theorie des eaux courantes', Mém. prés. par div. savants á l' Acad. Sci., Paris 23, No. 1, p. 1.

Boussinesq, J.: 1897, Théorie de l'écoulement tourbillonnant et tumultueux des liquides dans les lits rectilignes á grande section, I-II, Gauthier-Villars, Paris.

Bumba, V., Howard, R., and Smith, S. F.: 1964, Carnegie Inst. of Washington Year Book 63, 6.

Bumba, V.: 1967, Rendiconti della Scuola Internazionale di Fisica "E. Fermi," 39 Corso, 77.

Bumba, V. and Obridko, V. N.: 1969, Solar Phys. 6, 104.

Bumba, V. and Obridko, V. N.: 1970, Solar Phys. 14, 80.

Busse, F. H.: 1970, Astrophys. J. 159, 629.

Busse, F. H.: 1973, Astron. Astrophys. 28, 27.

Caccin, B., Falciani, R., Moschi, G., and Rigutti, M.: 1970, Solar Phys. 13, 33.

Chandrasekhar, S.: 1961, Hydrodynamic and Hydromagnetic Stability, Clarendon Press, Oxford.

Cocke, W. J.: 1967, Astrophys. J. 150, 1041.

Condon, E. U. and Shortley, G. H.: 1951, The Theory of Atomic Spectra, Cambridge University Press, Cambridge.

Conti, P. S.: 1968, Astrophys. J. 152, 657.

Cowling, T. G.: 1951, Astrophys. J. 144, 272.

Deinzer, W. and Stix, M.: 1971, Astron. Astrophys. 12, 111.

Deubner, F. L.: 1972, Solar Phys. 22, 263.

Dicke, R. H.: 1964, Nature 202, 432.

Dicke, R. H.: 1970a, IAU Colloq. 4 , 289.

Dicke, R. H.: 1970b, Ann. Rev. Astron. Astrophys. (ed. by L. Goldenberg), Annual Reviews; California, p. 297.

Dicke, R. H.: 1971, Phys. Rev. Letters, 27, 210.

Dicke, R. H.: 1972, Astrophys. J. 171, 331.

Dicke, R. H. and Goldenberg, H. M.: 1967, Phys. Rev. Letters 18, 313.

Dittmer, P. H.: 1975, Solar Phys. 41, 1975.

Dupree, A. K. and Henze, W.: 1972, Solar Phys. $27,271$.

Durney, B. R.: 1968a, J. Atmospheric Sci. 25, 372.

Durney, B. R.: 1968b, J. Atmospheric Sci. 25, 771.

Durney, B. R.: 1970, Astrophys. J. 161, 1115.

Darney, B. R.: 1971, Astrophys. J. 163, 353.

Durney, B. R.: 1972a, Solar Phys. 26, 3.

Durney, B. R.: 1972b, Solar Wind, Proc. of the Asilomar Conf., p. 282 (ed. by C. P. Sonnet, P. J. Coleman, Jr., and J. M. Wilcox), NASA, Washington.

Durney, B. R.: 1974a, Astrophys. J. 190, 211.

Durney, B. R.: 1974b, Solar Phys. 38, 301.

Durney, B. R.: 1976, Astrophys. J. 204, 589.

Durney, B. R. and Roxburgh, I. W.: 1971, Solar Phys. 16, 3.

Durney, B. R. and Skumanich, A.: 1968, Astrophys. J. 152, 255.

Falciani, R., Rigatti, M., and Roberti, G.: 1974, Solar Phys. 35, 277.

Fossat, E.: 1975, Thesis, Univ. of Nice (unpublished).

Fossat, E. and Ricort, G.: 1973, Solar Phys. 28, 311.

Fricke, K.: 1968, Z. Astrophys. 68, 317.

Fricke, K. and Kippenhahn, R.: 1970, Ann. Rev. Astron. Astrophys. 10, 45. 
Gierasch, P.: 1974, Astrophys. J. 190, 199.

Gilman, P. A.: 1972, Solar Phys. 27, 3.

Gilman, P. A.: 1975, J. Atmospheric Sci. 32, 1331.

Goldreich, P. and Schubert, G.: 1967, Asırophys. J. 150, 571.

Grevesse, N.: 1968, Solar Phys. 5, 159.

Hansen, R. T., Hansen, S. F., and Loomis, H. G.: 1969, Solar Phys. 10, 135.

Hauge, Ö. and Engvold, O.: 1968, Astrophys. Letters, 2, 235.

Heard, W. B.: 1973, Astrophys. J. 186, 1065.

Heard, W. B. and Veronis, G.: 1973 (unpublished).

Henze, W. and Dupree, A. K.: 1973, Solar Phys. 33, 425.

Herring, J. R.: 1963, J. Atmospheric Sci. 20, 325.

Herring, J. R.: 1964, J. Atmospheric Sci. 21, 277.

Herring, J. R.: 1969, Phys. Fluids 12, 39.

Hill, H. A. and Stebbins, R. T.: 1975a, Ann. N.Y. Acad. Sci. 262, 472.

Hill, H. A. and Stebbins, R. T.: 1975b, Astrophys. J. 200, 484.

Hill, H. A., Clayton, P. D., Patz, D. L., Healy, A. W., Stebbins. R. T., Oleson, J. R., and Zanoni, C. A.: 1974, Phys. Rev. Letters, 33, 1497.

Hill, H. A., McCullen, J. D., Brown, T. M., and Stebbins, R. T.: 1975 (unpublished).

Hill, H. A., Stebbins, R. T., and Brown, T. M.: 1976, Proc. of the Fifth Int. Conf. on Atomic Masses and Fundamental Constr., Paris, France (in press).

Howard, L. N., Moore, D. W., and Spiegel, E. A.: 1967, Nature 214, 1297.

Howard, R.: 1971, Solar Phys. 16, 21.

Howard, R. and Harvey, J.: 1970, Solar Phys. 12, 23.

Howard, R. and Yoshimura, H.: 1976, this volume, p.19.

Iroshnikov, R. S.: 1969, Astron. Zh. 46, 97 (translated in Soviet Astron. 13 (1969), 73).

Kaufman, P.: 1972, Solar Phys. 23, 178.

Kippenhahn, R.: 1963, Astrophys. J. 137, 664.

Köhler, H.: 1970, Solar Phys. 13, 3.

Köhler, H.: 1973, Astron. Astrophys. 25, 467.

Kobrin, M. M. and Korshunov, A. I.: 1972, Solar Phys. 25, 1972.

Kraft, R.: 1967, Astrophys. J. 150, 551.

Kraft, R.: 1969, in Stellar Astronomy, vol. 2 (ed. by H. Y. Chiu, R. Warasila and J. Remo), Gordon and Breach, New York.

Kraft, R.: 1970, in Otto Struve Memorial Volume (ed by G. Herbig), p. 385.

Ledoux, P., Schwarzschild, M., and Spiegel, E. A.: Astrophys. J. 133, 184.

Leighton, R. B.: 1966 (unpublished).

Leighton, R. B.: 1969, Astrophys. J. 156, 1.

Lerche, I. and Parker, E. N.: 1972, Astrophys. J. 176, 213.

Livingston, W. C.: 1969, Solar Phys. 9, 448.

McIntosh, P. S.: 1975, Report UAG $40\left(H_{\alpha}\right.$ Synoptic Charts of Solar Activity for the Period of Skylab Observations. March 1973-March 1974).

Mattig, W. and Nesis, A.: 1974, Solar Phys. 36, 3.

Mehltretter, J. P.: 1971, Solar Phys. 16, 253.

Modisette, J. L.: 1967, J. Geophys. Res. 72, 1521.

Ness, N. F. and Wilcox, J. M.: 1966, Astrophys. J. 143, 23.

Newton, H. W. and Nunn, M. L.: 1951, Monthly Notices Roy. Astron. Soc. 111, 413.

Noyes, R. W., Ayres, T. R., and Hall, D. N. B.: 1973, Solar Phys. 28, 343.

Osaki, Y.: 1970, Monthly Notices Roy. Astron. Soc. 131, 407.

Pai, S. 1956, Viscous Flow Theory, Vol. 1, Laminar Flow, Van Nostrand, New York, pp. 39-40.

Parker, E. N.: 1955, Astrophys. J. 122, 293.

Parker, E. N.: 1971, Astrophys. J. 164, 491.

Parker, E. N.: 1973a, Astrophys. J. 186, 643.

Parker, E. N.: 1973b, Astrophys. J. 186, 665.

Parker, E. N.: 1975, Astrophys. J. 198, 205.

Richardson, R. S. and Schwarzschild, M.: 1953, Academia Lincei, Conv. 11, p. 228.

Roberts, P. H.: 1974, Solar Wind Three, p. 231 (ed. by C. T. Russell), published by Inst. of Geophys. and Planetary Phys., UCLA.

Roberts, P. H. and Stix, M.: 1972, Astron Astrophys. 18, 453.

Ross, J. E. and Aller, L. H.: 1974, Solar Phys. 36, 11.

Roxburgh, I. W.: 1964, Icarus 3, 92.

Roxburgh, I. W.: 1970, IAU Colloq. 4. 
Roxburgh, I. W.: 1974, Astrophys. Space Sci. 27, 419.

Rüdiger, G.: 1974, Astron. Nachr. 295, 229.

Rüdiger, G.: 1976, Solar Phys. (in press).

Rutten, R. J.: 1973, Solar Phys. $28,347$.

Sakurai, T.: 1966, Publ. Astron. Soc. Japan 18, 174.

Sakurai, T.: 1975, Monthly Notices Roy. Astron. Soc. 171, 35.

Schatzman, E.: 1962, Ann. Astrophys. 25, 18.

Shaviv, G. and Salpeter, E. E.: 1973, Astrophys. J. 184, 191.

Simon, G. W. and Noyes, R. W.: 1972, Solar Phys. 26, 8.

Simon, G. W. and Weiss, N. O.: 1968, Z. Astrophys. 69, 435.

Skumanich, A.: 1955, Astrophys. J. 121, 408.

Skumanich, A.: 1972, Astrophys. J. 171, 565.

Spiegel, E. A.: 1964, Astrophys. J. 139, 959.

Spiegel, E. A.: 1965, Astrophys. J. 141, 1068.

Spiegel, E. A.: 1968, in Highlights of Astronomy (ed. by L. Perek), D. Reidel Publishing Co., Dordrecht.

Spiegel, E. A.: 1971, Ann. Rev. Astron. Astrophys. 9, 330 (ed. by L. Goldberg).

Spiegel, E. A. and Zahn, J. P.: 1970, Comments Astrophys. Space Phys. 2, 178.

Starr, V. P. and Gilman, P.: 1965, Astrophys. J. 141, 1119.

Steenbeck, M. and Krause, F.: 1969, Astron. Nachr. 291, 49.

Stenflo, J. O.: Solar Phys. 36, 495.

Stix, M.: 1974, Astron. Astrophys. 37, 121.

Stix, M.: 1976a, this volume, p. 367.

Stix, M.: 1976b (submitted to Astron. Astrophys.).

Strittmatter, P. A.: 1969, Ann. Rev. Astron. Astrophys. 7, 665.

Švestka, Z.: 1968a, Solar Phys. 4, 18.

Švestka, Z.: IAU Symp. 35, 287.

Tuominen, J.: 1955, Z. Astrophys. 27, 145.

Unno, W.: 1961, Publ. Astron. Soc. Japan 13, 276.

Vandakurov, Yu. V.: 1975a, Solar Phys. 40, 3.

Vandakurov, Yu. V.: 1975b, Solar Phys. (in press).

Veronis, G., 1966, Tellus 18, 67.

Vickers, G. T.: 1971, Astrophys. J. 163, 363.

Wallerstein, G. and Conti, P. S.: 1969, Ann. Rev. Astron. Astrophys. 7, 99.

Wallerstein, G., Herbig, G. H., and Conti, P. S.: 1965, Astrophys. J. 141, 610.

Ward, F.: 1965, Astrophys. J. 141, 534.

Weber, E. J. and Davis, L., Jr.: 1967, Astrophys. J. 148, 217.

Weiss, N. O.: 1964, Monthly Notices Roy. Astron. Soc. 128, 225.

Weiss, N. O.: 1965, Observatory 85, 37.

Wilcox, J. M. and Howard, R.: 1968, Solar Phys. 5, 564.

Wilcox, J. M. and Howard, R.: 1970, Solar Phys. 13, 251.

Wilcox, J. M. and Ness, N. F.: 1965, J. Geophys. Res. 70, 5793.

Wilcox, J. M. and Ness, N. F.: 1967, Solar Phys. 1, 437.

Wilcox, J. M., Schatten, K. H., Tanenbaum, A. S., and Howard, R.: 1970a, Solar Phys. 14, 225.

Wilcox, J. M., Schatten, K. H., Tanenbaum, A. S., and Howard, R.: 1970b, Solar Phys. 14, 255.

Wilson, O. C.: 1966a, Astrophys. J. 144, 695.

Wilson, O. C.: 1966b, Science 151, 1487.

Wolff, C. L.: 1974a, Astrophys. J. 193, 721.

Wolff, C. L.: 1974b, Astrophys. J. 194, 489.

Wolff, C. L.: 1975, Solar Phys. 41, 297.

Yoshimura, H.: 1971, Solar Phys. 18, 417.

Yoshimura, H.: 1972a, Solar Phys. 22, 20.

Yoshimura, H.: 1972b, Astrophys. J. 178, 863.

Yoshimura, H.: 1975a, Astrophys. J. Suppl. 29, 467.

Yoshimura, H.: 1975b, Astrophys. J. 201, 740.

Yoshimura, H.: 1976a, this volume (Discussion).

Yoshimura, H.: 1976b (unpublished).

Yoshimura, H. and Kato, S.: 1971, Publ. Astron. Soc. Japan 23, 57.

Young, R.: 1974, J. Fluid Mech. 63, 695.

Zahn, J. P. 1974, IAU 59, 185.

Zahn, J. P.: 1975, Coll. Internat. d'Astr. de Liege (in press). 


\section{DISCUSSION}

Roxburgh: (1) Is it not possible that $\Delta F$ is large in the bulk of the convective zone but becomes small in the surface layers where $\Delta \nabla T$ becomes large since with a large $\Delta \nabla T$ a small circulation could possibly destroy the $\Delta F$ ?

(2) In your calculation you assume that the turbulent model can be applied all the way to the surface of the convective zone, but in the surface layers radiative transport becomes dominant and even if $\Delta F / F$ is large for the convective flux, the convective flux itself goes to zero at the surface so $\Delta F$ could also go to zero. In the top $1000 \mathrm{~km}$ where radiative transfer is dominant the $\Delta F$ can be wiped out.

Durney: (1) It is possible, However, one cannot help thinking that somehow the main effect of the constraint imposed by rotation on convection is only differential rotation and not large pole-equator differences in flux inside the convection zone. We do not know at present how a large $\Delta \boldsymbol{\Omega}$ can be achieved with a small $\Delta F$.

(2) I think that it is unlikely that a large $\Delta F$ can be wiped out in the top $1000 \mathrm{~km}$ with no manifestations at the surface. Differential rotation could be a surface phenomenon but then no large $\Delta F$ 's are needed, but this appears to be in contradiction with dynamo models.

Schröter: One of your alternative solutions out of the dilemma is the suggestion of the existence of a large pole-equator flux difference within the convective zone. Would you expect from such a fact an observable difference in the appearance (size, contrast, life-time, etc.) of the solar granulation pattern between pole and equator?

Durney: I think it is unlikely. If a large pole-equator difference does indeed exist inside the solar convection zone, it completely disappears in the surface layers and would not affect, I think, the solar granulation.

Weiss: We can place some constraints on the variation of energy flux with latitude. In the radiative zone $\Delta F$ must be very small, any significant variation of flux on an equipotential surface will rapidly be eliminated by mass motions. At the photosphere we observe no appreciable variation. On the other hand, a significant variation in the deep convective zone might be eliminated near the surface just as the supergranular temperature variations are below the limit observation.

Durney: It is possible, and to answer this question with certainty is an important problem that remains to be solved. However, the Sun could satisfy the constraints imposed by rotation on convection by processes of which we do not have yet any idea.

Chvojková: During the maximum of a solar cycle high magnetic fields of about $10^{3} \mathrm{G}$ should be expected just below the photosphere. Thus at the top of the convection zone there should exist a layer in which the magnetic pressure prevailed the kinetic one, the motion would become nonisotropic, the field would act against most of the movements of the described mechanism. Hence, the differential rotation should be most probably somewhat smaller during cycle maxima. Is it so or is the layer $H^{2} / 8 \pi>\frac{1}{2} \rho V^{2}$ too thin and insignificant for affecting the result?

Durney: If we accept Parker's point of view, the solar cycle is generated in the lower part of the convection zone and the magnetic flux tube rises at about the Alfvénic speed, thus very rapidly in the surface layers. Large toroidal fields waiting to be dissipated or carried away by the solar wind should be present below the photosphere and could indeed influence the Sun's differential rotation. I think that there are observations confirming this.

Mestel: I seem to recall that Biermann postulated that there should not be any large latitude variations in flux, and then derived the necessity for circulation from momentum balance. Kippenhahn then used this circulation to construct the departure from uniformity of rotation.

Durmey: I think that Biermann postulated a different rate of momentum exchange between the direction parallel and perpendicular to gravity. He then proved that conservation of angular momentum precludes solid rotation. 